\section{DOE/ER/B3910-DT2}

UNIVERSITY OF CALIFORNIA

Los Angeles
Apperdix

DOE/ER/13910--T2

DE93 002223

\title{
Evaporation and Burning of a Spherical Fuel Droplet \\ in a Eniform Convective Flowfield
}

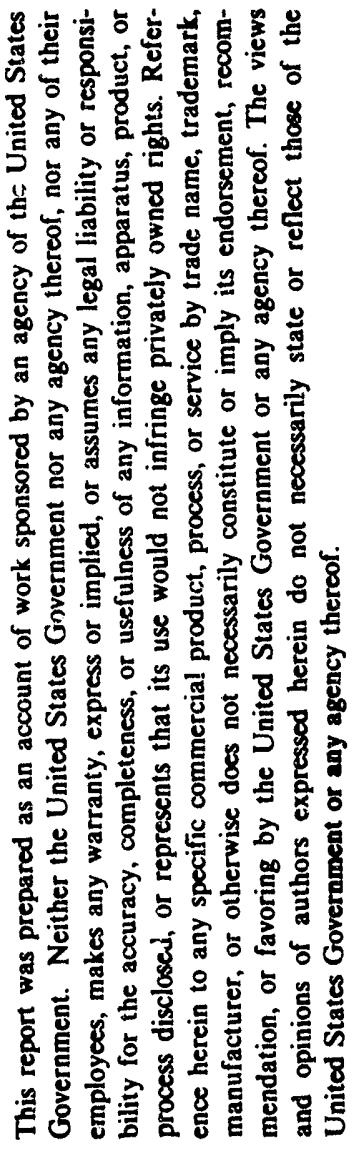

\author{
FG O3-88ER 13910 \\ A dissertation submitted in partial satisfaction of the \\ requirements for the degree Doctor of Philosophy \\ in Mechanical Engineering \\ by
}

Kevork Madooglu

1992

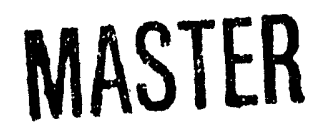

BISTRIBUTION OF THIS DOCUMENT IS UNLIMITED 
The dissertation of Kevork Madooglu is approved.

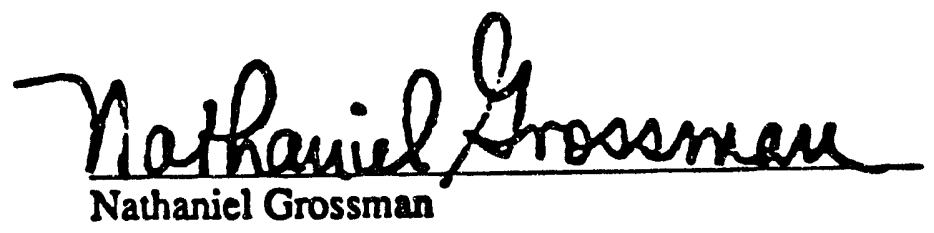

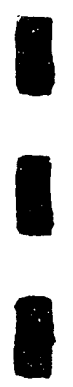

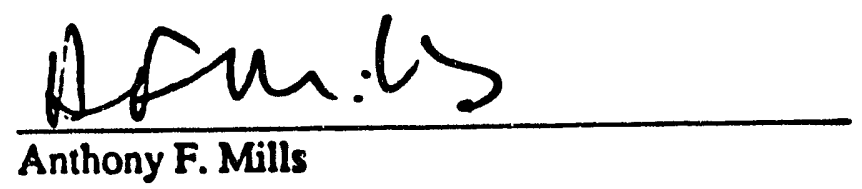

Pele A. Honker 2

Peter A. Monkewitz

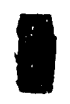

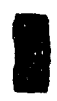

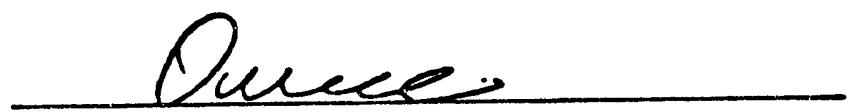

Owen I. Smith

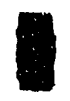

Ann R. Kuragoj

Ann R. Karagozian, Committee Chair

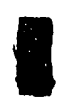

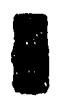

University of California, Los Angeles

1992

ii 
To my family:

my parents,

Roza, Tamar, Hirac, Kevork, Barig, Patil, and Chris. 


\section{Table of Contents}

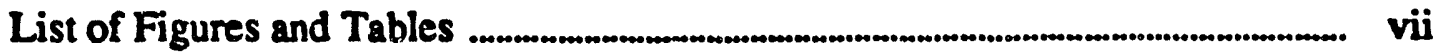

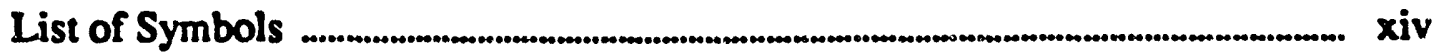

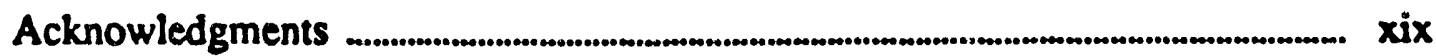

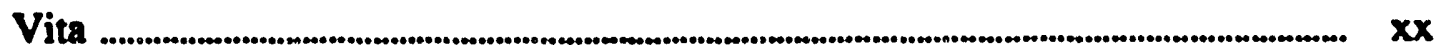

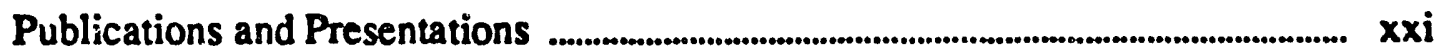

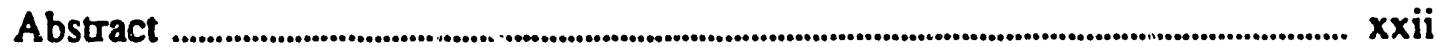

CHAPTER 1. Introduction and Background .......................................................... 1

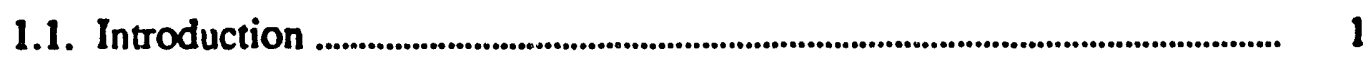

1.2. Sphericity of the Drop ........................................................................................ 3

1.3. Empirical Correlations for Evaporation Rates from Single Drops

in a Convective Environment ..................................................................................

1.4. Previous Theoretical Work on the Evaporation and Buming of a

Single Drop in a Convective Environment .............................................................. 7

1.5. Finite-Rate Chemistry and Ignition .......................................................... 11

CHAPTER 2. Steady-State Evaporation and Buming ............................................ 17

2.1. Formulation and Method of Solution .......................................................... 17

2.1.1. External Gaseous Flowfield .................................................................. 18

2.1.2. Internal Liquid Flow ...................................................................... 20

2.1.3. Gaseous Boundary Layer Analysis .............................................. 21

2.1.4. Calculation of Drag and Evaporation Rate ........................................ 29

2.2. Results and Discussion ............................................................................. 31 


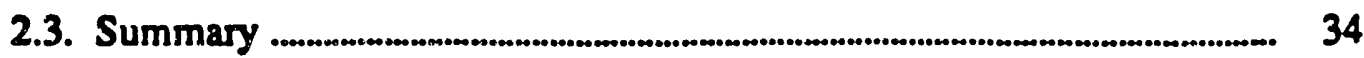

CHAPTER 3. Transient Evaporation and Buming …............................................. 36

3.1. Transient Heating of the Droplet Interior ........................................................... 36

3.1.1. Streamline Coordinates ........................................................... 36

3.1.2. Initial and Boundary Conditions .......................................................... 40

3.2. Coupling of Gas and Liquid Phase Solutions ........................................ 41

3.3. Results and Discussion ............................................................................... 42

3.3.1. Effective Transfer Number ....................................................................... 42

3.3.2. Comparison with Empirical Correlations ............................................ 43

3.4. Summary ................................................................................................................................. 46

CHAPTER 4. Single-Step, Finite-Rate Chemistry ..................................................... 48

4.1. Chemistry ....................................................................................................................... 48

4.2. Boundary Layer Equations .................................................................................. 49

4.3. Results and Discussion ....................................................................................... 52

4.3.1. Activation Energy .................................................................................... 53

4.3.2. Fast versus Finite-Rate Chemistry ....................................................... 54

4.4. A Criterion for Ignition .................................................................................... 56

4.5. An Improved Representation of Chemistry ................................................. 57

4.6. Summary ......................................................................................................... 59

CHAPTER 5. "Exact" Variation of Gas Properties in the Boundary Layer

5.1. Boundary Layer Equations .................................................................................... 62

5.2. Thermophysical Properties .................................................................................... 65

5.3. Results and Discussion ......................................................................................... 68

5.3.1. "Exact" versus "Model" Properties ..................................................... 68 
5.3.2. Comparison with Experiments

5.4. Summary ..................................................................................................................... 76

CHAPTER 6. Conclusions ..................................................................................... 78

6.1. Boundary Layer versus Fully Numerical Modeling ......................................... 78

6.2. Steady-State Burning: Flame Position and Droplet Histories .................. 80

6.3. An Effective Transfer Number for the Entire Droplet Lifetime

6.4. Finite-Rate versus Infinite-Rate Chemistry ....................................................... 82

6.5. "Exact" Variation of Gas Properties ................................................................... 84

References ................................................................................................................................. 86 


\section{List of Figures and Tables}

Figure 1-1. Schematic illustration of the dependence of the maximum temperature on the relevant Damkohler nurnber for diffusion flames after Williams (1985).

Figure 2-1. Schematic diagram of the flowfield associated with a liquid fuel droplet in a convective environment.

Figure 2-2. Characteristics of the boundary layer and reaction zone near the surface of the liquid droplet.

Figure 2-3. Variation of pressure distribution along the surface of a rigid

sphere.

Figure 2-4. Variation of dimensionless vorticity along the surface of a

rigid sphere.

Figure 2-5. Variation of drag coefficient with free stream Reyncids

number for a rigid sphere.

Figure 2-6. Predicted velocity, temperature, and concentration profiles in the boundary layer of the buming droplet at an angular location $\theta=60^{\circ}$ from the stagnation point. 
Figure 2-7. Comparison of evaporation rate and skin friction coefficient with the predictions of Rangel and Fernandez-Pello (1984) at $p_{-}=1 \mathrm{~atm}$, $T_{\infty}=293 \mathrm{~K}, \mathrm{Re}_{\infty}=120, \mathrm{M}_{\infty}=0.01$.

Figure 2-8. Effect of the freestream Mach number, $M_{\infty}$, on the skin friction coefficient and evaporation rate per unit area along the burning droplet surface at $p_{\infty}=1 \mathrm{~atm}, T_{-}=293 \mathrm{~K}, R \epsilon_{\infty}=50$.

Figure 2-9. Predicted variation in drag coefficient of the droplet with freestream Reynolds number, for both evaporating and buming droplets. Comparison is made with the experimental data of Yuen and Chen (1976) for an evaporating droplet, and with the "standard" curve for the drag on a solid sphere.

Figure 2-10. Predicted variation in mass transfer rate of the droplet with Reynolds number, for evaporating and burning droplets. Comparison is made with Renksizbulut-Yuen and Froessling/Ranz-Marshall correlations for an evaporating droplet.

Figure 2-11. Predicted droplet histories (radius and effective Reynolds number), for evaporating and burning droplets. Comparison is made with the experimental data of Renksizbulut and Yuen (1983a), using the data on drag coefficient from Yuen and Chen (1976).

Figure 2-12. Predicted flame shapes about spherical droplet for two different Reynolds numbers. 
Figure 3-1. Illustration of the orthogonal streamline coordinate system $(\xi, \Psi, \omega)$. 104

Figure 3-2. The functions $j_{1}(\psi)$ and $J_{2}(\psi)$.

105

Figure 3-3. Predicted temporal variation of the nondimensional evaporation rate, compared with various empirical correlations.

Figure 3-4. Predicted temporal change in droplet radius, compared with the predictions of Prakash and Sirignano (1980).

Figure 3-5. Predicted temporal change in surface temperature, compared with the predictions of Prakash and Sirignano (1980) at $\theta=90^{\circ}$. 108

Figure 3-6. Predicted temporal change in droplet radius, compared with the predictions of Sanders and Dwyer (1987). 109

Figure 4-1. Predicted temperature, velocity, and species mass fraction profiles at an angular distance $\theta=60^{\circ}$ from the upstream stagnation point of the droplet.

Figure 4-2. Effect of the activation energy on predicied temperature profiles within the boundary layer.

Figure 4-3. Effect of the activation energy on predicted fuel vapor and oxygen mass fraction profiles within the boundary layer.

Figure 4-4. Variation of the local skin friction coefficient and mass blow- 
ing rate along the droplet surface for different activation energies.

Figure 4-5. Temperature and fuel mass fraction profiles, predicted by Models 1 and 2, at an angular distance $\theta=60^{\circ}$ from the upstream stagnation point.

Figure 4-6. Variation of the local and mass blowing rate and skin friction cuefficient aling the droplet surface, as predicted by Models 1 and 2 .

Figure 4-7. Variation of the nondimensional integrated reaction rate along the droplet surface for different activation energies.

Figure 4-8. Temperature and fuel mass fraction profiles, predicted by Models 2 and 3, at an angular distance $\theta=60^{\circ}$ from the upstream stagnation point.

Figure 4-9. Variation of the local and mass blowing rate and skin friction coefficient along the droplet surface, as predicted by Models 2 and 3 .

Figure 4-10. Velocity profiles predicted by Models 2 and 3, at an angular distance $\theta=60^{\circ}$ from the upstream stagnation point.

Table 5-1. Force constants for the Lennard-Jones potential model (from Edwards et al. (1979)).

Table 5-2. Collision integrals for the Lennard-Jones potential model (from Edwards et al. (1979)). 
Figure 5-1. Variation of specific heat, Prandt number, $p \mu$, temperature, and species mass fractions across the boundary layer at an angular distance $\theta=60^{\circ}$ from the upstream stagnation point.

Figure 5-2. Variation of specific heat, Prandtl number, $\rho \mu$, temperature, and species mass fractions across the boundary layer at an angular distance $\theta=60^{\circ}$ from the upstream stagnation point.

Figure 5-3. Temperature and fuel mass fraction profiles at an angular distance $\theta=60^{\circ}$ from the upstream stagnation point, predicted by Model 4, and by Model 3 for two different average specific heats.

Figure 5-4. Variation of the local mass blowing rate along the droplet surface, as predicted by Model 4, and by Model 3 for two different average specific heats.

Figure 5-5. Variation of the local skin friction coefficient along the droplet surface, as predicted by Model 4, and by Model 3 for two different average specific heats.

Figure 5-6. Variation of fuel, oxygen and nitrogen Lewis numbers across the boundary layer at an angular distance $\theta=60^{\circ}$ from the upstream stagnation point.

Figure 5-7. Temperature and fuel mass fraction profiles at an angular distance $\theta=60^{\circ}$ from the upstream stagnation point, predicted by Model 4 . and by Model 3 for two different effective Lewis numbers. 
Figure 5-8. Variation of the local mass blowing rate along the droplet surface, as predicted by Model 4, and by Model 3 for two different effective Lewis numbers.

Figure 5-9. Variation of the local skin friction coefficient along the droplet surface, as predicted by Model 4, and by Model 3 for two different effective Lewis numbers.

Figure 5-10. Comparison of temperature and fuel mass fraction profiles at an angular distance $\theta=60^{\circ}$ from the upstream stagnation point, predicted by Models 1 to 4 .

Figure 5-11. Variation of the local mass blowing rate along the droplet surface, as predicted by Models 1 to 4.

Figure 5-12. Variation of the local skin friction coefficient along the droplet surface, as predicted by Models 1 to 4 .

Figure 5-13. Predicted variation in mass transfer rate from the burning droplet with Reynolds number at $700 \mathrm{~K}$. Comparison is made with the experimental observations of Law and Williams (1972), and the Froessling/Ranz-Marshall correlation.

Figure 5-14. Predicted variation in mass transfer rate from the buming droplet with Reynolds number at $300 \mathrm{~K}$. Comparison is made with the experimental observations of Law and Williams (1972), and the Froessling/Ranz-Marshall correlation. 
Figure 5-15. Predicted droplet histories (radius and Reynolds number) for a buming droplet at $300 \mathrm{~K}$. Comparison is made with the experimental data of Law and Williams (1972), using the data on drag coefficient from Yuen and Chen (1976).

Table 6-1. Some of the previous theoretical work on convective droplet evaporation and burning. 


\section{List of Symbols}

A Hill's vortex strength

A Frequency factor in the Arthenius relation

a Speed of sound

B Transfer number

$C_{D} \quad$ Drag coefficient $\left(\equiv 2 F_{D} /\left(\pi R^{2} p_{\infty} u_{\infty}^{2}\right)\right)$

$C_{i} \quad$ Molar concentration of the $i-t h$ gas species

c Specific heat at constant pressure for gas

D Mass diffusivity

Da Damkohler number

E Dimensionless activation energy $\left(\equiv E_{\mathrm{a}} /\left(\mathrm{R}_{\mathrm{u}} \mathrm{T}_{\mathrm{e}}\right)\right)$

E $\quad$ Activation energy

Unit vector

F Dimensionless velocity $\left(\equiv u / u_{e}\right)$

FD Total drag force exerted on droplet

f Dimensionless stream function defined by (2.20)

Stoichiometric fuel-oxidizer ratio

G Composite variable defined by (2.16)

xiv 
H Total enthalpy $\left(=h+u^{2} / 2\right)$

h Enthalpy

$h_{v} \quad$ Latent heat of vaporization

J Total number of chemical reactions

$J_{1}, J_{2}$ Dimensionless functions defined by (3.13)

K Composite variable defined by (2.17)

$\mathbf{K}_{\mathbf{i}} \quad$ Mass fraction of the $i$-th gas species

k Chemical reaction rate coefficient

M Mach number

$\dot{\mathbf{m}} \quad$ Rate of evaporation of droplet .

N Total number of species involved

P Pressure

Pe Peclet number $(\equiv 2 \mathrm{uR} / \alpha)$

Pr Prandu number $(\equiv \mu /(p \alpha))$

$\dot{\mathbf{Q}} \quad$ Rate of heat transfer

$9 \quad$ Heat release per unit mass of fuel

R Droplet radius

$\mathbf{R}_{\mathbf{i}} \quad$ Gas constant for the $i-$ th gas species

Ro Initial radius of droplet

$\mathbf{R}_{\mathbf{u}} \quad$ Universal gas constant

I Radial coordinate 
Dimensionless radial coordinate $(=r / R)$

r. Local radius of droplet cross-section

Re Reynolds number $(\equiv 2 p u R / \mu)$

Sc Schmidt number $(\equiv \mu /(\rho D))$

T Temperature

To Initial liquid-phase temperature

$\left(u_{r}, u_{\theta}\right) \quad r-$ and $\theta$-components of velocity vector $u$

$(u, v)$ Velocity components tangential and normal to droplet surface

$\mathbf{W}_{\mathbf{i}} \quad$ Molecular weight of the $\mathrm{i}-\mathrm{th}$ gas species

$\dot{w}_{i} \quad$ Mass rate of production for the $i-t h$ gas species

We Weber number $\left(\equiv 2 p u^{2} R / \sigma\right)$

$(x, y)$ Coordinates tangential and normal to droplet surface

y $\quad$ Local flame position relative to droplet surface

Greck Symbols

a Thermal diffusivity

B Pressure gradient parameter

4. Dimensionless parameter defined in (4.14)

$\gamma \quad$ Ratio of specific heats

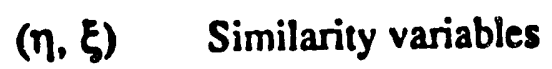

$\theta$ Dimensionless temperature ( $\left.\equiv \mathrm{T} / \mathrm{T}_{\mathrm{e}}\right)$

$\theta$ Coordinate indicating angular distance along droplet 
6. Separation point

$\lambda$ Thermal conductivity

$\mu \quad$ Dynamic viscosity

v Kinematic viscosity

p Density

- Surface tension; force constant for the Lennard-Jones potential

$\tau \quad$ Dimensionless diffusion time scale

Velocity potential

$\Psi \quad$ Stream function for gas-phase motion

$\Psi \quad$ Stream function for liquid-phase motion

i Dimensionless stream function for liquid-phase motion

$\Omega_{D}, \Omega_{\mu}$ Collision integrals for the Lennard-Jones potential

\section{Subscripts}

$\begin{array}{ll}\text { e } & \text { Conditions at edge of external boundary layer } \\ \text { f } & \text { Fuel or flame } \\ \text { i } & \text { Gas phase } \\ \text { I } & \text { i-th gas species } \\ \text { n } & \text { Liquid phase } \\ \text { - } & \text { Nitrogen } \\ \text { p } & \text { Oxidizer }\end{array}$

xvii 
Freestream gas conditions

xviii 


\section{ACKNOWLEDGMENTS}

Much credit for this dissertation must go to my advisor, Professor Ann Karagozian, not only for her most valuable academic guidance, but also for her constant support and encouragement throughout my Ph.D. work at UCLA.

I would like to thank my committee members, Professors Mills, Monkewitz, Smith and Grossman for improving the quality of this study through their helpful comments and suggestions. Thanks are also due to Professor Marble or California Institute of Technology for sharing his vast insight and experience of many years.

This research has been supported in part by the U.S. Department of Energy under Grant DE-FG03-88-ER13910 and by NASA Ames/Dryden Flight Research Center under Grant NCC 2-374.

xix 


\author{
ABSTRACT OF THE DISSERTATION \\ Evaporation and Burning of a Spherical Fuel Droplet \\ in a Uniform Convective Flowfield \\ by \\ Kevork Madooglu \\ Doctor of Philosophy in Mechanical Engineering \\ University of Califomia, Los Angeles, 1992 \\ Professor Ann R. Karagozian, Chair
}

An analytical/numerical model is developed for the evaporation and buming of a spherical fuel droplet in a subsonic crossflow. The extemal gaseous flowfield is represented using an approximate compressible potential-flow solution, while the internal flowfield of the droplet is represente: by the classical Hill's spherical vorex. This allows a numerical solution for the external boundary layer, from which the droplet's effective drag coefficent, rate of mass loss, size, and the shape of the diffusion flame with infinitely fast chemical reaction kinetics are determined. Subsequently, the quasi-steady model with uniform liquid temperature is extended to examine the effects of the transient heating of the droplet interior. Time-dependent calculations are performed with updated droplet Reynolds numbers and updated surface temperatures. Comparisons of model predictions with experimental data are made. To examine the effects of finite-rate chemical reaction kinetics, a one-step formulation of the combustion mechanism is integrated into the gaseous boundary layer equations. Simplifying assumptions for the variation of gas properties, 
commonly used in combustion calculations, are subjected to an examination as to their degree of accuracy. For this purpose, the droplet model is extended to account for the variation of gas properties with temperature and gas composition within the boundary layer. Comparisons are made between the predictions obtained from the different models developed in this study, as well as with existing experimental data.

xxiii 


\section{VITA}

November 14, 1960

1986

1987-1988

1987

1988-1989

1989-1990

1990

1990-1992

1991-1992
Born, Istanbul, Turkey

Diplom-Ingenieur, Mechanical Engineering University of Karlsruhe

Karlsruhe, Federal Republic of Germany

Graduate Student Researcher University of California, Los Angeles

Los Angeles, California

M.Sc., Mechanical Engineering University of California, Los Angeles

Los Angeles, California

Consulting Engineer

Hersh Acoustical Engineering, Inc.

Chatsworth, California

Graduate Student Researcher

University of California, Los Angeles

Los Angeles, California

Teaching Assistant

University of California, Los Angeles

Los Angeles, California

Graduate Student Researcher University of California, Los Angeles Los Angeles, California

Teaching Assistant University of California, Los Angeles

Los Angeles, California 


\section{PUBLICATIONS AND PRESENTATIONS}

K. Madooglu, I. Catton, F. Ghebremichael and J. Goodman, "Two-Phase Flow in a Magnetogasdynamic Duct", Proceedings of SPIE, vol. 87I, pp. 2-9 (1988).

K. Madooglu, I. Catton, F. Ghebremichael, J. Goodman, "Two-Phase Flow in a Nuclear MHD Generator", ANS Proceedings, 1988 National Heat Transfer Conference, pp. 427-434 (1988).

K. Madooglu, A.R. Karagozian, "Buming of a Spherical Fuel Droplet in a Uniform Subsonic Flowfield," Paper No. 89-65, Presented at the 1989 Fall Meeting of the Western States Section/Combustion Institute (1989).

K. Madooglu, I. Catton, F. Ghebremichael, J. Goodman, "Condensation Phenomena in Quasi-One Dimensional Magnetogasdynamic Flow," Chemical Engineering Communications, Vol. 87, pp. 107-122 (1990).

- K. Madooglu, A.R. Karagozian, "Transient Heating and Burning of a Fuel Droplet in a Uniform Flowfield," Paper GF7, Presented at the 43rd Annual Meeting of the Division of Fluid Dynamics/American Physical Society (1990). 


\section{CHAPTER 1 INTRODUCTION AND BACKGROUND}

\subsection{Introduction}

Over the past few decades, the widespread use of liquid sprays in combustion systems has led to increased research interest in tive study of single droplet evaporation and combustion in a convective environment, as pointed out in a number of comprehensive review articles, such as Faeth (1977), Law (1982) and Sirignano (1983). Especially in the 1980 's, extensive effor has been directed toward the analytical and computational modeling of this problem, which is complicated by the transient, multi-phase, multi-dimensional nature of the transfer processes involved.

In many practical combustor situations, Reynolds numbers associated with both gas and liquid phases are not larger than $O(100)$. This "intermediate" range of Reynolds numbers makes a fully numerical treatment of the gas and liquid flow.fields seem necessary. However, the transient fully-numerical solution for the lifetime of a burning fuel droplet requires immense computational effor. Dwyer (1989) reports that computing times associated with the fully-numerical solution of droplet evaporation and burning are of the order of 2 hours of CPU time on a Cray computer. Furthermore, these fully numerical models almost always involve various simplifications in each calculation, such as assumption of constant gas properties, 
neglect of liquid phase motion or heat transfer, and/or restriction of the range of Reynolds, Peclet and Mach numbers involved (Dwyer and Sanders $(1986,1988)$ ).

Facing these difficulties associated with full computer simulation even more severely in the seventies, Faakash and Sirignano $(1978,1980)$ developed an analytical model suitable for high Reynolds numbers both in the gas and liquid phases, which ultimately gave reasonable results for an evaporating fuel droplet at Reynolds numbers of $O(100)$. Encouraged by and building on the work of Prakash and Sirignanc: (1980), the present study aims at developing an analytical/numerical model for single droplet combustion in a uniform convective flowfield which will involve the significant features of the problem without requiring large computational times. In particular, we wish to examine the effects of chemical reaction, which was not considered by Prakash and Sirignano (1980), employing both infinite and finite rate representations of chemical reaction kinetics for an envelope flame around the droplet. We also wish to estimate the error introduced by simplifications in the evaluation of thermophysical properties, which is commonly done in combustion analyses to reduce computational times. For this purpose, the exact variation of gas properties within the boundary layer will have to be taken into account. Reducing the com. putational effort significantly, the boundary-layer approach employed in the present study will enable such an analysis within very reasonable computational times. The representation of compressibility in the flow approaching the droplet provides a Mach-number dependent analysis for the gaseous boundary layer which is sufficiently accurate for free stream Mach numbers $M_{\infty}<0.3$. 


\subsection{Sphericity of the Drop}

If the Weber number for a liquid drop in forced convection or in gravitational motion, defined as

$$
W e=\frac{2 p_{-} u^{2} R}{\sigma}
$$

$p_{\infty}$ and $u_{\infty}$ being the density and relative velocity of the gaseous flowfield around the droplet, $R$ the droplet radius and $\sigma$ surface tension, is above a critical value, the drop gets deformed and, eventually, breaks up into smaller drops. Based on experimental observations, Eisenklam (1961) gives the critical Weber number as $W e_{c r}=13$ for drops in forced convective flow, and as $W e_{c r}=22$ for freely falling drops. It can be shown in a straightforward manner that this Weber number limit corresponds to an upper limit for the product of the Reynolds and Mach numbers $\left(\operatorname{Re}_{\infty} M_{\infty}\right)$ in terms of gas and liquid properties, i.e.

$$
\operatorname{Re}_{\infty} M_{\infty} \leq 13 \frac{\sigma}{\mu_{\infty} a_{\infty}} \text {. }
$$

where $\mu_{\infty}$ is the viscosity and $a_{\infty}$ the speed of sound in the free stream. Eq. (1.2) thus defines a regime of sphericity for a drop in forced convective flow. For the cases studied within the framework of this thesis, the drop can be assumed nearly spherical according to this criterion.

Clift, Grace and Weber (1978) give a similar shape regime curve in terms of the Reynolds number and the Eotvos number $E O=4\left(\rho_{1}-\rho_{\infty}\right) g R^{2} / \sigma$ for gravitational motion of drops. In the Reynolds number regime that we are interested in, i.e. $50 \leq R e \infty \leq 200$, this plot gives roughly Eo $\leq 0.5$, which yields, with $W e_{a}=22$ for gravitational motion, 


$$
\operatorname{Re}_{\infty} M_{\infty} \leq 44 \frac{4 p_{1} g R^{2}}{H_{\infty} 2_{\infty}}
$$

\subsection{Empirical Correlations for Evaporation Rates from Single Drops in a Con-} vective Environment.

Experimental research on the evaporation of single drops in a convective environment dates back to the 1930 's. As early as 1938, Froessling (1938) was able to obtain an empirical correlation for the evaporation of single suspended nitrobenzene, naphtalene and water drops at room temperature and atmospheric pressure. The correlation proposed by Froessling for the rate of evaporation from a drop is

$$
\dot{\mathrm{m}}=\dot{\mathrm{m}}_{\mathrm{ss}}\left(1+0.276 \mathrm{Sc}^{1 / 3} \operatorname{Re}_{-1 / 2}^{1 / 2}\right)
$$

where $S c$ is the Schmidt number of the surrounding gas, and $\dot{\mathrm{m}}_{\mathrm{SS}}$ denotes the rate of evaporation from a drop in the theoretical case of spherically-symmetric, diffusion controlled evaporation which would occur in the absence of forced convection and gravitation. $\dot{\mathrm{m}}_{\mathrm{ss}}$ is calculated as (e.g. see Kuo (1986))

$$
\dot{\mathrm{m}}_{\mathrm{sB}}=4 \pi R \rho_{s} D_{s} \ln (1+B) \text {. }
$$

where $p_{s}$ and $D_{s}$ are, respectively, the mass density and the mass diffusivity of the gas at the surface of the drop, and $B$ is the so-called Spalding transfer number, defined as

$$
B=\frac{K_{f, 8}-K_{f,-\infty}}{1-K_{f, 8}} .
$$

$\mathbf{K}_{\mathbf{f}}$ heing the mass fraction of the fuel vapor in the surrounding gas mixture.

Froessling's correlation (1.4) was obtained from experiments performed within 
the range of Reynolds numbers $2<R e_{\infty}<800$.

Ranz and Marshall (1952) were able to confirm Froessling's results after experiments on suspended water drops at atmospheric pressure and $358 \mathrm{~K} \leq \mathrm{T}_{\infty} \leq 494 \mathrm{~K}$. They reported a slightly different coefficient than Froessling's:

$$
\dot{\mathrm{m}}=\dot{\mathrm{m}}_{\mathrm{se}}\left(1+0.30 S \mathrm{c}^{1 / 3} \operatorname{Re}_{\mathrm{e}^{1 / 2}}\right) .
$$

This type of correlation has become known as the "Ranz-Marshall-correlation," and widely used in practical applications. Ranz and Marshall's data was taken at $10 \leq \operatorname{Re}_{\infty} \leq 200$.

Froessling's and Ranz and Marshall's experiments did not involve chemical reaction. Spalding (1953) used a porous metal sphere filled with liquid fuel to simulate the buming of a fuel drop in a convective environment. The experiments were conducted at Reynolds numbers $800 \leq R_{-} \leq 4000$, and for transfer numbers $0.6 \leq B \leq 5$. Spalding proposed the correlation

$$
\dot{\mathrm{m}}^{\prime \prime}=\frac{\dot{\mathrm{m}}}{4 \pi \mathrm{R}^{2}}=0.53 \frac{\mu}{2 R} B^{3 / 5} R e^{1 / 2}
$$

for buming drops at these high Reynolds numbers.

The porous sphere technique was also used by Agoston et al. (1958). These: authors gave for ethanol and methanol burning at high temperatures $\left(2200 \mathrm{~K} \leq \mathrm{T}_{-} \leq 2900 \mathrm{~K}\right)$ the correlation

$$
\dot{\mathrm{m}}=\dot{\mathrm{m}}_{\mathrm{ss}}\left(1+0.24 \mathrm{Re}_{\mathrm{s}}^{\mathrm{k}}\right)
$$

to represent their data which contained a high degree of scatter. Furthermore, $\operatorname{Pr}=1$ was assumed in this correlation.

A third technique, measurements from freely falling drops, was employed by 
EisenkJam et al. (1966) and Natarajan and Brzustowski (1970). Eisenklam et al. (1966) proposed correlations for both evaporating and buming drops, but these correlations will not be mentioned here since the experiments were carried out at very low Reynolds numbers (Re $\left.e_{\infty} \leq 10\right)$. Natarajan and Brzustowski (1970), on the other hand, approximated their data with the same type of correlation for both evaporation and burning, i.e.

$$
\dot{\mathrm{m}}^{\prime \prime}=0.73 \frac{\mu_{m}}{\operatorname{Pr} R} \frac{B}{1+B} \operatorname{Re}_{m}^{y_{m}^{*}}
$$

where the subscript $m$ implies that average quantities were employed for gas properties. These average values as well as the transfer number B were defined separately for evaporation and buming. Natarajan and Brzustowski's data was taken at $4.4 \mathrm{~atm} \leq \mathrm{p}_{\infty} \leq 41.85 \mathrm{~atm}, 10 \leq \mathrm{Re}_{\mathrm{m}} \leq 20,000$ and $\mathrm{T}_{\infty}=589 \mathrm{~K}$, for drops of the size $0.1 \mathrm{~mm} \leq \mathrm{R} \leq 1 \mathrm{~mm}$.

Finally, Renksizbulut and Yuen (1983a) measured evaporation rates from suspended $n$-Heptane drops in the absence of chemical reaction. These authors suggested a modified version of the Froessling/Ranz-Marshall type correlation:

$$
\dot{m}^{\prime \prime}=\frac{\mu_{m}}{\operatorname{Pr} R} \frac{B}{(1+B)^{0.7}}\left(1+0.285 \operatorname{Pr}^{1 / 3} \operatorname{Re}_{m}^{1 / 2}\right),
$$

where $\mathrm{Pr}=\mathrm{Sc}$ was assumed. The data was taken at atmospheric pressure, for $511 \mathrm{~K} \leq \mathrm{T}_{\infty} \leq 723 \mathrm{~K}_{,} 78 \leq \mathrm{Re}_{\mathrm{m}} \leq 1974,0.9 \leq \mathrm{B}_{\mathrm{m}} \leq 2.79$, and $0.82 \mathrm{~mm} \leq \mathrm{R} \leq 3.18 \mathrm{~mm}$.

The $\mathrm{Re}^{1 / 2}$-dependence in all of these correlations suggests that for large Reynolds numbers heat and mass transfer take place across a laminar boundary layer adjacent to the droplet surface. 


\subsection{Previous Theoretical Work on the Evaporation and Burning of a Single Drop in a Convective Environment}

Until the late 1970's, theoretical studies of droplet vaporization and combustion were restricted to the spherically symmetric case of a droplet evaporating into an infinite quiescent environment even though empirical correlations for convective effects on single droplet evaporation, based on experimental observations, existed since the days of Froessling (1938) and Ranz and Marshall (1952). Earlier work on spherically symmetric droplet evaporation and combustion had suggested that the

evaporation process should obey the so-called $\mathrm{d}^{2}$-law for the surface regression rate if it could be considered quasi-steady (see, e.g., review article by $\mathbf{A}$. Williams (1973)). This was verified by the zero-gravity experiments of Okajima and Kumagai (1974). The fully numerical solution of the transient problem by Hubbard et al. (1975) confirmed both the validity of the $\mathrm{d}^{2}$-law for the later part of droplet lifetime and the importance of the initial transient period of droplet heat-up, the length of which is subject to ambient conditions and fuel properties.

The effect of a convective flowfield over an evaporating drop is twofold: the evaporation process is altered not only by the gas-phase convection at the droplet surface but also by the liquid-phase motion and heat transfer within the droplet. Harper and Moore (1968) examined the motion of a drop in another fluid, assuming the Reynolds numbers to be large enough for a boundary layer type of approach to be valid, but small enough for surface tension to keep the drop in nearly spherical shape. They showed that for this case, except for the viscous liquid and gas boundary layers near the drop surface, the flowfield would be described by potential flow outside, and by Hill's spherical vortex inside the drop. The strength of the voriex in 
relation to the free stream velocity could be determined imposing a condition for shear stress continuity at the drop surface.

LeClair et al. (1972) calculated the velocity at the surface of a water sphere in air flow using different approaches, and compared their results with experimental observations. For free stream Reynolds numbers of $\mathrm{Re}_{-}=\mathrm{O}(100)$ and dropet radia of $R \leq 0.5 \mathrm{~mm}$ the results obtained from the boundary-layer model as employed by Harper and Moore (1968) were very close to results obtained from numerical simulation, and these were both in good agreement with experimental results.

Numerical solutions for drops or bubbles moving in fluids were also presented by Rivkind and Ryskin (1976) and Rivkind et al. (1976) for the whole range between $\mu_{1} / \mu_{\infty} \rightarrow 0$ (spherical bubble) to $\mu_{1} / \mu_{\infty} \rightarrow \infty$ (solid sphere). The results of both LeClair et al. (1972) and Rivkind and associates (1976) show that for $\mathrm{Re}_{\infty}=O(100)$ the streamline pattem inside the drop resembles very much that of Hill's vorrex although the vorticity distribution may be somewhat different. Another significant result of the above mentioned authors' numerical work is that for these moderate free stream Reynolds numbers, i.e. $\operatorname{Re}_{\infty}=\mathrm{O}(100)$, there will be only a very small region near the downstream stagnation point where a secondary vonex, if any, can be formed due to the negative shear stress in the wake region downstream of separation.

The mass transfer process in a droplet experiencing convection from a surrounding fluid was examined in connection with solute extraction from falling droplets by Kronig and Brink (1951), Johns and Beckmann (1964) and Brignell (1975) among others. Both Kronig and Brink's (1951) and Brignell's (1975) work is based on two basic assumptions: (i) the circulatory motion within the droplet is described by the Hill's vortex solution, and (ii) mass flux of the extracted substance is normal 
to the streamsurfaces of the Hill's vortex. The latter assumption is based on a reasoning by Kronig and Brink (1951) that the time required by a liquid particle to pass once around a closed streamline to its starting point is negligibly smaller than the time of mass diffusion. Then, the concentration of the dissolved substance can be assumed to be the same at any point of the streamline, but varies from one streamline to the other. Thus, spatially, concentration can be considered to be a function of the streamline coordinate only, and the differential equation for species conservation can be simplified accordingly. Johns and Beckmann (1966) solved the problem numerically, showing that the solution for large Peclet numbers $(\mathrm{Pe}>100)$ approaches that of Kronig and Brink (1951) which, essentially, is an infinite Peclet number solution.

The significance of the work related to solute extraction from moving droplets for droplet evaporation is that both heat and mass transfer in a droplet with internal circulation are described by the same type of diffusion equation. Thus, the findings of the above-mentioned authors can be applied to our problem by simply replacing solute concentration with liquid temperature in the diffusion equation as the property of interest. Pan and Acrivos (1968) examined the heating of a body of circulating liquid and showed that, as a parallel to the solute concentration in the above mentioned case, the liquid temperature $T_{1}$ is given by $T_{1}=T_{1}(\psi)+O\left(P e^{-1}\right)$ for $P e>1$, where $\psi$ is the streamline coordinate.

Building on these findings by previous investigators, Prakash and Sirignano (1978) developed a model for a drop experiencing evaporation, internal circulation and unsteady heating. Later, they coupled this liquid-phase solution with a gaseous boundary layer solution around the droplet (Prakash and Sirignano (1980)). Thus, essentially, the analytical model of Prakash and Sirignano (1980) contains four flow 
regions: an external gaseous region of inviscid, incompressible flow, a gaseous boundary layer adjacent to the droplet surface solved using integral methods, a viscous layer in the liquid phase solved assuming a perturbation solution for the velocity, and an inviscid liquid core where the motion is described by Hill's spherical vorex. Based on an argument related to the comparison of the characteristic diffusion times involved, the authors assumed that the only unsteady process in the problem is the heat transfer in the liquid core, where $T_{1}=T_{1}(\psi)+O\left(P e^{-1}\right)$ for $P e>1$, as mentioned above. The heat transfer has to be considered unsteady for many practical situations. For example, for heavy fuels at high pressures and temperatures the heat-up time for an evaporating droplet can be about as long as its entire lifetime.

Despite the fact that the range of Reynolds numbers in this problem raises questions about the use of the boundary layer approach, and that the assumed isotherm pattern within the droplet is established only after an initial development (see numerical work of Dwyer et al. (1984)), Prakash and Sirignano (1980) reported satisfactory agreement of their results with existing empirical correlations, such as the RanzMarshall (1952) correlation and the Spalding (1953) correlation which is based on data taken at higher Reynolds numbers, i.e. $800 \leq \operatorname{Re} \leq 4000$. In the following years, Lara-Urbaneja and Sirignano (1981) extended this model for studies of multicomponent droplets, whereas Tong and Sirignano (1982) developed a more simplified model involving an approximate analytical solution for the heat transfer in the liquid phase in an effort to obtain results applicable to spray combustion calculations.

Using a similar approach, Rangel and Femandez-Pello (1984) examined the effects of a diffusion flame (i.e. infinitely fast chemical kinetics) on the evaporation process of a circulating droplet. They included the effects of natural convection in their work, maintaining the axisymmetric nature of the problem - which 
corresponds to the burning of a dropiet falling under the influence of gravity, for instance. However, they did not consider the transient heating of the droplet. The reacting boundary layer was solved using a Blasius-type series solution.

Finally, it should be mentioned that the convective droplet evaporation and burning problem has also been modelled fully numericaly in the mid and late $1980^{\circ}$ s. For a drop evaporating in the absence of chemical reaction, Renksizbulut and Yuen (1983b), Dwyer and Sanders (1984), Renksizbulut and Haywood (1988), Haywood

et al. (1989), Huang and Ayyaswamy (1990) and Chiang et al. (1992) performed fully numerical calculations, the latter four being unsteady' calculations. The latter three also took into account variation of thermophysical properties with temperature and mixture composition, instead of representing it through simplifying assumptions, as commonly done in combustion analyses. Later, Dwyer and Sanders (1986) included chemical reaction in their computations.

\subsection{Finite-Rate Chemistry and Ignition}

Ignition is defined as "a transition from a nonreactive to a reactive state in which extemal stimuli lead to thermochemical runaway followed by a rapid transition to self-sustained combustion" (Kuo (1986)). It can be introduced either by external sources such as sparks, pilot flames, hot wires, or it can occur spontaneously, i.e. by the mixture of reactants reaching the thermodynamic conditions necessary and sufficient for chemical reaction. In the present study we will restrict ourselves to the latter ignition mechanism, spontaneous ignition, also termed "selfignition" or "thermal ignition" in the literature. 
Self-ignition of hydrocarbon fuels in the form of liquid droplets in air proceeds through a series of steps which can be roughly outlined as follows (A. Williams (1990)): (i) The surface temperature of the droplet increases, primarily by conduction, and the vapor pressure of the fuel increases; (ii) the fuel vapor mixes with the oxygen in the air and the temperature of the combustible fuel vapor-air mixture increases; (iii) the composition of the mixture becomes within the inflammability limits for the particular fuel-air mixture and the temperature increases so that it exceeds the ignition temperature; (iv) at this point the rate of the hydrocarbonoxygen chain reaction exceeds the critical limit and ignition occurs. Obviously, there are a number of open questions associated with this "critical limit," such as what the mixture composition and temperature necessary for ignition are, or when and where ignition starts.

The complex, transient nature of the self-ignition process makes it necessary that a more or less sophisticated representation for the hydrocarbon-oxygen chain reaction be employed to study it. The simplest representation for chemical reaction kinetics, a diffusion flame with infinitely fast chemistry, is not suitable for ignition studies. The simplest reaction rate model accounting for finite rate chemistry is a one-step chemical reaction with an Arthenius-type reaction rate expression, i.e.

$$
k(T)=A_{8} \exp \left[-\frac{E_{2}}{R_{u} T}\right] .
$$

where $A_{t}$ is the "frequency factor", $E_{d}$ is the activation energy, $R_{u}$ is the universal gas constant, and $\mathbf{T}$ is the temperature of the gas mixture. This type of representation, however, also poses problems, for instance, in terms of determining an "ignition temperature," the temperature at which ignition will occur. As readily seen from eq. (1.12), the reaction rate $k$, according to the Arrhenius type of representation, will be 
nonzero for all temperatures. One higher stage of chemistry modeling is, then, a multi-step representation of the chemical reaction that is closer to reality.

Problerns like this, and, in general, the complex nature of the ignition process, make the detailed examination, interpretation and prediction of ignition phenomena very difficult. Furthermore, experimental observations are equally difficult to perform due to the extremely short characteristic times associated with this transient process. Nevertheless, a significant amount of research effort has been devoted to the study of ignition (and extinction) phenomena, and certain criteria that can be used to predict ignition (and extinction) have been developed.

As mentioned above, ignition and extinction are essentially transient processes. However, one may assume that ignition can be achieved only if the steady-state equations possess a solution describing combustion. Therefore, analyses based on quasi-steady flow can still provide information that is relevant to ignition and extinction.

For examining finite-rate influences on diffusion flames and for developing ignition (or extinction) criteria, a number of different approaches have been employed by different investigators. Perturbation methods combined with simplified chemical kinetics have been useful in establishing a better understanding of the nature of the physical processes involved. Two types of perturbation approaches are utilized for this purpose: Damkohler-number asymptotics and activation-energy asymptotics. The Damkohler number is a similarity group that is defined as the ratio of a characteristic diffusion time to a characteristic reaction time, or, reaction rate to diffusion rate. This means that for small Damkohler numbers, i.e. $\mathrm{Da} \rightarrow 0$, one has nonreacting flow, the "frozen-flow" limit, whereas $\mathrm{Da} \rightarrow \infty$ implies practically infinite chemical reaction rate, the "equilibrium" limit. 
If one plots the maximum temperature $T_{m}$ versus the Damkohler number $\mathrm{Da}$, as shown qualitatively in Fig. 1-1, one obtains a characteristic S-shaped curve (Williams (1985)). The lower branch of the curve represents the frozen flow regime, while the upper branch characterizes the near-equilibrium flow regime. The turning points of the curve determine the critical Damkohler number for extinction, $\mathrm{DaE}_{\mathrm{E}}$, below which no chemical reaction is possible, and the critical Damkohler number for ignition, $\mathrm{Da}_{1}$, above which one has a fast-chemistry diffusion fiame. When one moves towards left on the upper branch by decreasing $\mathrm{Da}_{1}$ there will be a jump in the termperature upon reaching $\mathrm{Da}_{E}$, i.e. abrupt extinction will take place. Similarly coming from the frozen-flow regime of low Damkohler numbers, abrupt ignition will occur upon reaching the ignition Damkohler number $D_{1}$. The fact that ignition and extinction conditions fall near the turning points of the S-shaped curve makes their computation difficult. Usually, rather laborious numerical integrations are needed to locate the comers (Williams (1971)).

Obviously, the regime $\mathrm{Da}_{E}<\mathrm{Da}<\mathrm{Da}$ Is likely to be unstable and, therefore, not significant for diffusion flames. Williams (1971) points out the difficulty of establishing combustion conditions experimentally that would correspond to points on the middle branch.

In the Damkohler-number asymptotics, $\mathrm{Da}$ is treated as a large parameter, which corresponds to the case of a flame approaching the limit of infinitely fast chemical reaction kinetics. Damkohler-number asymptotics, combined with onestep Arrhenius-type reaction kinetics, was employed by Friedlander and Keller (1963), Fendell (1965), Kassoy and Williams (1968) for studies related to reactionzone thickness and to obtain profiles with continuous gradients for temperature and mass fractions, which is nor possible if one assumes infinitely fast chemistry. 
Damkohler-number asymptotics also provides the possibility of analyzing the small Damkohler-sumber regime. However, analyses of ignition and extinction, i.e. computations in the vicinity of the turning points of the S-curve, cannot be performed using Damkohler-number asymptotics. For this type of studies, large activationenergy asymptotics is more useful for it is valid for all Damkohler numbers.

Large activation-energy asymptotics was applied first by Linan (1974) on diffusion flames. In this analysis "large" activation energies are assumed, which really means that the ratio of the activation energy $E_{a}$ to the thermal energy $\left(R_{u} T\right)$ is much larger than unity (see equation (1.12)). The most significant result of this type of analysis was that conclusions were possible with respect to different reaction regimes and the existence of critical Damkohler numbers for ignition and extinction, i.e. the turning points of the S-shaped curve. Law $(1975,1978)$ employed large activation-energy asymptotics combined with a one-step Arrhenius-type reaction to determine critical ignition and extinction Damkohler numbers for a single droplet burning in a quiescent atmosphere. Krishnamurty (1976) and Femandez-Pello and Law (1982) examined convective ignition by large activation-energy asymptotics at the stagnation point of a droplet. These authors determined critical Damkohler numbers for ignition using matched asymptotic expansions. Using the analytical methods established by these authors, Rangel and Fernandez-Pello (1986) calculated critical ignition Damkohler numbers for a droplet in a mixed-convective flowifield, neglecting the effects of transient droplet heating and liquid-phase motion.

Numerical integration of the conservation equations has been employed by a number of investigators through the use of known reaction rates in one step (Lorell et al. (1956)), two-step (Jain and Mukunda (1969)), or multi-step (Liu and Libbj (1970)) representations for the combustion of different fuels. The main problem 
with the examination of ignition and extinction phenomena, however, is the lack of detailed knowledge of the physical parameters involved in the chemical reaction kinetics. Kashiwagi and Summerfield (1972) introduced an ignition criterion that enabled predictions based on experimental observations. For the boundary-layer flow of a hot oxidizing gas over a solid fuel surface, these authors postulated that ignition would occur when the integrated reaction rate through the boundary layer was larger than a certain value $c^{*}$, i.e.

$$
\int_{0}^{\infty}(\text { reaction rate }) d y \geq c^{*} \text {. }
$$

Here, $y$ is the coordinate normal to the fuel surface. A value for $c^{*}$ was selected by the authors based on the experimental ignition criterion of a minimum detectable light density, seen by photomultipliers viewing the boundary layer. 


\section{CHAPTER 2 \\ STEADY-STATE EVAPORATION AND BURNING}

\subsection{Formulation and Method of Solution}

As mentioned in the previous chapter, the present study aims at developing an analytical/numerical model for the combustion of a single droplet in a uniform convective flowfield. Since one objective of this modeling effort is to avoid large computational times, a boundary-layer type of analysis is employed, which, of course, is valid only for moderate to high Reynolds number regimes. Species diffusion and the presence of a diffusion flame with infinitely fast chemical kinetics are represented in the gaseous boundary layer adjacent to the droplet surface. The extemal, gaseous flowfield about the droplet is solved by an approximate analytical method, and this solution is to drive the external gaseous boundary layer flow and the circulating flow. in the droplet interior. A schematic description of the ieatures of the present model is shown in Fig. 2-1.

In this chapter, a uniform liquid-phase temperature is assumed. Since earlier work such as that of Prakash and Sirignano (1980) has shown that the liquid.phase heating is essentially unsteady in the very early periods of droplet lifetime, calculations presented in this chapter would apply to larger droplets in the later stages of evaporation and burning, when a uniform liquid temperature is reached. 


\subsubsection{External Gaseous Flowfield}

The extemal inviscid gas flow about the spherical droplet is represented by a compressible potential flow solution. Defining the velocity potential $\phi(r, \theta)$ for axisymmetric fluid motion, the potential equation for steady, irrotational, isentropic flow of a perfect gas reduces to (Van Dyke (1975)):

$$
\left[1-\frac{M_{\omega}^{2}}{2}(\gamma-1)[u \cdot u-1]\right] \nabla^{2} \phi=\frac{M^{2}}{2}\left[u_{\tau} \frac{\partial}{\partial t}+\frac{u_{\theta}}{T} \frac{\partial}{\partial \theta}\right] u \cdot u .
$$

We note that the isentropic flow assumption requires any chemical reaction to take place within the viscous boundary layer adjacent to the spherical droplet.

The boundary conditions for equation (2.1) are given by the requirements that the flow is uniform far from the sphere and that the normal velocity at the sphere's surface is zero. For "slightly compressible" flow, i.e. small free-stream Mach number $\left(M_{-}^{2} \ll 1\right)$, the solution of equation (2.1) can be expanded into the form (Van Dyke (1975)):

$$
\phi\left(r, \theta ; M_{\infty}^{2}\right)=\phi_{0}(r, \theta)+M_{\infty}^{2} \phi_{1}(r, \theta)+O\left(M_{\infty}^{4}\right) .
$$

Since it readily follows from equation (2.1) that all the effects of compressibility are $O\left(M_{1 \infty}^{2}\right)$, the zeroth order term $\phi_{0}(r, \theta)$ in equation $(2.2)$ is given by the solution of the homogeneous Laplace equation $\nabla^{2} \phi_{0}=0$, with the boundary conditions

$$
\phi_{0} \rightarrow-u_{-} r \cos \theta \text { as } r \rightarrow \infty \text {, }
$$

and

$$
\frac{\partial \phi_{0}}{\partial t}=0 \text { at } r=R \text {. }
$$

This, of course, recovers the well-known solution for incompressible potential flow 
over a sphere (Panton (1984)):

$$
\phi_{0}(r, \theta)=-u_{0} R\left[1+\frac{1}{2 r^{3}}\right] r \cos \theta \text {. }
$$

where a dimensionless radial coordinate is introduced as $\hat{\mathrm{r}}=\mathbf{r} / \mathbf{R}$.

For the first-order term $\phi_{1}(\hat{r}, \theta)$, one has from equations (2.1) and (2.2)

$$
\nabla^{2} \phi_{1}=\frac{1}{2 R^{2}}\left[\frac{\partial \phi_{0}}{\partial \hat{r}} \frac{\partial}{\partial \dot{r}}+\frac{1}{\hat{r}^{2}} \frac{\partial \phi_{0}}{\partial \theta}\right]\left[\left[\frac{\partial \phi_{0}}{\partial \dot{r}}\right]^{2}+\left[\frac{1}{\hat{r}} \frac{\partial \phi_{0}}{\partial \theta}\right)^{2}\right]
$$

The boundary conditions for the velocity potential $\phi(r, \theta)$ have already been satisfied by the zeroth order solution $\phi_{0}(\hat{r}, \theta)$, so that $\phi_{1} \rightarrow 0$ as $\hat{r} \rightarrow \infty$ and $\frac{\partial \phi_{1}}{\partial \hat{r}}=0$ at $\hat{r}=1$. Substituting $\phi_{0}(\hat{r}, \theta)$ from $(4)$ into $(5)$, one obtains the explicit governing equation for $\phi_{1}:$

$$
\nabla^{2} \phi_{1}=-\frac{u_{\infty}}{R}\left[\left[\frac{9}{8} \tilde{r}^{-1}-\frac{9}{2} \tilde{r}^{-7}+\frac{81}{32} \tilde{r}^{-10}\right] \cos \theta+\left[\frac{15}{8} \tilde{r}^{-1}-\frac{3}{2} \tilde{r}^{-7}+\frac{15}{32} \tilde{r}^{-10}\right] \cos 3 \theta\right]
$$

which can be solved by separation of variables, yielding the expression

$$
\begin{aligned}
\phi_{1}(\hat{r}, \theta)= & -u_{\infty} R\left[\left[\frac{53}{240} \tilde{r}^{-2}+\frac{81}{440} \tilde{r}^{-4}-\frac{5}{16} \tilde{r}^{-5}+\frac{203}{4224} \tilde{r}^{-8}\right] \cos \theta\right] \\
& +u_{\infty} R\left[\left[\frac{3}{16} \tilde{r}^{-2}-\frac{27}{88} \tilde{r}^{-4}+\frac{3}{16} \tilde{r}^{-5}-\frac{15}{1408} \tilde{r}^{-8}\right] \cos 3 \theta\right] .
\end{aligned}
$$

The potential-fiow solution for the extemal gas flow is thus obtained to order $M_{\text {s. }}^{4}$. which can be regarded as sufficiently accurate for the low subsonic flow regime.

Assuming the viscous layer to be "thin", i.e. the boundary-layer approximation to be valid, the velocity at the edge of the viscous layer, $u_{e}(\theta)$, is obtained from 
differentiation of the velocity potential $\phi$, which gives

$$
\frac{u_{e}(\theta)}{u_{\infty}}=\frac{3}{2} \sin \theta-\frac{83}{220} M^{2} \sin \theta\left[1-\frac{1215}{664} \sin ^{2} \theta\right]+O\left(M_{\infty}^{4}\right) \text {. }
$$

For isentropic inviscid flow, the temperature, pressure, and Mach number at the boundary layer edge can be computed from freestream values in a straightforward manner. The use of the boundary layer approximation also requires the evaporation rate from the droplet, or the radial velocity at droplet surface, to be small. In Section 2.1.3 it will be shown that this requirement is fulfilled.

\subsubsection{Internal Liquid Flow}

The gas flow over a liquid drop induces a liquid-phase motion within the drop through momentum transfer at the gas-liquid interface. Harper and Moore (1968) show that the internal recirculating flow of a droplet in a convective environment can be represented by the well-known Hill's spherical vortex solution if one neglects the existence of small counterflow vortices near the downstream stagnation point driven by the vortical flowfield beyond the separation point. In the high Reynolds number limit, the streamsurfaces for this type of flow are described by the stream function

$$
\psi=\psi_{0}-\frac{1}{2} A R^{4} \hat{r}^{2}\left(1-\hat{r}^{2}\right) \sin ^{2} \theta .
$$

In the above equation, $A$ denotes the vortex strength, which is a constant throughout the inviscid liquid-flow region. This unknown vortex strength $A$ is to be determined through the coupling of the gaseous and liquid-phase solutions.

The velocity components of the liquid flow can be obtained in a straightforward manner, so that as a first approximation, the velocity at the edge of an intemal 
viscous layer, $u_{i}(\theta)$, is represented by the magnitude of the Hill's vortex velocity vector at $\mathbf{r}=\mathbf{R}$ :

$$
u_{i}(\theta)=A R^{2} \sin \theta,
$$

indicating that the presence of the internal viscous layer is actually neglected here. According to the boundary layer approximation, the error introduced by this assumption will be $O\left(\operatorname{Re}_{i}^{-1 / 2}\right)$ for surface velocity. Similarly, the error for the surface temperature will be $O\left(\mathrm{Pe}_{i}^{-1 / 2}\right)$. Brignell (1975) gives a lengthy and detailed discussion about the error introduced by the neglect of the viscous layer near the drop surface. In the analysis of Harper and Moore (1968), the velocity at the surface is taken to be only a small perturbation of its value at the edge of the viscous liquid layer. Prakash and Sirignano (1978) show that this is a valid assumption while the same may not be true for vorticity, since the difference in vorticity between the surface and the edge of the viscous layer may not be negligible. However, as will be seen in the "Results and Discussion" section, the overall effect of liquid motion on the transfer processes in the gaseous boundary layer is relatively insignificant. Thus, the emphasis being placed on overall evaporation and drag calculations within the scope of the present study, the surface motion is approximated by the inviscid inner flow solution at the limit $\mathbf{r}=\mathbf{R}$. This reduces the required computational time for the solution of the coupled gas-liquid boundary layers considerably, since the (presenuly neglected) liçuid boundary layer solution is effectively obtained from calculation of one unknown parameter, namely the vortex strength $A$ that appears in the boundary conditions for the gas flow, as outlined in the following section of this chapter.

\subsubsection{Gascous Boundary Layer Analysis}


For the viscous gas flow adjacent to the droplet surface, the governing equations and boundary conditions account for the effects of chemical reaction, tangential surface motion, and mass blowing due to evaporation (and combustion) at the gas-liquid interface within the framework of a laminar axisymmetric boundary layer, the thickness of which is much smaller than the droplet radius. The burning process is represented by infinitely fast reaction kinetics, which shrinks the reaction zone effectively into a "flame sheet" of zero thickness within the boundary layer (the Burke-Schumann hypothesis), dividing it into two regions (see Fig. 2-2). The governing equations, written in surface coordinates $(x, y)$, are then (see e.g. Kuo (1986))

$$
\begin{gathered}
\frac{\partial}{\partial x}\left(r_{s} \rho u\right)+\frac{\partial}{\partial y}\left(r_{s} \rho v\right)=0 \\
\rho\left[u \frac{\partial u}{\partial x}+v \frac{\partial u}{\partial y}\right]=p_{c} u_{c} \frac{d u_{c}}{d x}+\frac{\partial}{\partial y}\left[\mu \frac{\partial u}{\partial y}\right] .
\end{gathered}
$$

and

$$
\rho\left[u \frac{\partial H}{\partial x}+v \frac{\partial H}{\partial y}\right]=\frac{\partial}{\partial y}\left[\frac{\mu}{\operatorname{Pr}} \frac{\partial H}{\partial y}\right]+\frac{\partial}{\partial y}\left[\frac{\mu}{\operatorname{Pr}}(\operatorname{Pr}-1) \frac{\partial}{\partial y}\left[\frac{u^{2}}{2}\right]\right]-q \dot{w}_{f} .
$$

where $r_{s}(x)$ is the radius of the cross-section of the sphere at $x$ and $H \equiv c_{p} T+u^{2} / 2$ is the total enthalpy. These equations, of course, are valid when the boundary layer thickness is considerably smaller than the droplet radius. The equations for conservation of species take the form

$$
\begin{aligned}
& \text { Region I, } y \leq y_{f}: \rho\left[u \frac{\partial K_{f}}{\partial x}+v \frac{\partial K_{f}}{\partial y}\right]=\frac{\partial}{\partial y}\left[\rho D \frac{\partial K_{f}}{\partial y}\right]+\dot{w}_{f} . \\
& \text { Region II, } y \geq y_{f}: \rho\left[u \frac{\partial K_{0}}{\partial x}+v \frac{\partial K_{0}}{\partial y}\right]=\frac{\partial}{\partial y}\left[\rho D \frac{\partial K_{0}}{\partial y}\right]+\frac{1}{r^{*}} \dot{w}_{f} .
\end{aligned}
$$


Here $K_{f}$ and $K_{0}$ denote the mass fractions of fuel and oxidizer, respectively, and $f^{*}$ is the stoichiometric fuel-oxidizer mass-consumption ratio, given by

$$
f^{*}=\frac{-\left(\partial K_{f} / \partial y\right)_{y_{f}}}{\left(\partial K_{0} / \partial y\right)_{y_{t}}} .
$$

All inert gases are assumed to have the same properties as the products of reaction and are treated together with the latter as one species. Furthermore, in the above equations, the simplifying assumption has been made that all the species involved in the problem have the same diffusion coefficient $D$.

The assumption of a thin flame sheet in the boundary layer introduces discontinuities in the enthalpy and concentration gradients at this location. By defining new composite variables for enthalpy and mass fraction,

$$
G \equiv \frac{H+r^{*} q K_{0}}{H_{e}+q_{0, e}}
$$

and

$$
K \equiv K_{0}-\frac{1}{f^{*}} K_{f}
$$

it is possible to eliminate these discontinuities as well as the source terms in equations (2.13), (2.14) and (2.15) (Burke and Schumann (1928), Chen and Toong (1964)). For laminar, axisymmetric boundary layer flows with variable gas properties, Lees (1956) suggests the following coordinate transformation that incorporates elements of the Mangler, Howarth-Dorodnitsyn and Levy transformations:

$$
\begin{gathered}
\xi(x)=\int_{0}^{x} \rho_{c} \mu_{c} v_{c} r_{g}^{2} d x, \\
\eta(x, y)=\frac{u_{c} r_{s}}{(2 \xi)^{1 / 3}} \int_{0} \rho d y .
\end{gathered}
$$


By defining a non-dimensional compressible stream function

$$
f \equiv \frac{\Psi(x, y)}{(2 \xi)^{1 / 2}}
$$

where

$$
\frac{\partial \Psi}{\partial y}=r_{3} p u_{0} \quad \frac{\partial \Psi}{\partial x}=-r_{s} p v_{0}
$$

one can transform the original governing equations into a set of ordinary differential equations, provided that there exist locally self-similar solutions, i.e. if $f, G$ and $K$ are functions of $\eta$ only. Thus finally introducing the non-dimensional velocity

$$
F(\eta) \equiv \frac{d f}{d \eta}=\frac{u}{u_{e}} .
$$

one then obtains the governing equations for $\rho \mu=$ const. and unity Lewis number,

$$
\begin{gathered}
F^{\prime \prime}(\eta)+f(\eta) F^{\prime}(\eta)-\beta(\xi)\left[\frac{p_{e}}{\rho}-F^{2}(\eta)\right]=0, \\
G^{\prime \prime}(\eta)+\operatorname{Pr} f(\eta) G^{\prime}(\eta)=\left(1-P_{T}\right) \frac{u_{e}^{2}}{B_{e}}\left[F(\eta) F^{\prime \prime}(\eta)+F^{\prime 2}(\eta)\right] . \\
K^{\prime \prime}(\eta)+\operatorname{Sc} f(\eta) K^{\prime}(\eta)=0,
\end{gathered}
$$

where $g_{e} \equiv H_{e}+r_{q}^{*} K_{0, e}$ and where primes denote differentiation with respect to the similarity parameter $\eta$. The assumption $\rho \mu=$ const. is widely used for mass-transfer boundary layers, non-reacting as well as reacting. As pointed out by Williams (1985), this assumption is valid as long as there are no abrupt changes in the average molecular weight of the gas mixture. Kuo (1986) suggests the approximation

$$
\frac{\rho_{\mu}}{\rho_{e} \mu_{e}}=\left[\left[\frac{\rho_{s} \mu_{s}}{\rho_{c} \mu_{e}}\right]_{0}\right]^{0.2}=\text { const. }
$$


which, for the cases considered in this study, corresponds to $0.93 \leq p \mu / p_{e} f_{e} \leq 0.99$. A thorough examination of the constant property assumption will be made in Chapter 5.

The problem is now transformed effectively into the well-known wedgeboundary-layer problem, with varying pressure gradients for varying angular positions. The "pressure gradient parameter" $\beta(\xi)$ in equation (2.22a) is defined as

$$
\beta(\xi)=\frac{2 \xi}{u_{c}} \frac{d u_{e}}{d \xi} .
$$

Since the pressure is constant across the boundary layer, the density ratio that appears in equation (2.22a), $p_{e} / p_{\text {, is equal to }} T / T_{e}$ and can be written as

$$
\frac{P_{e}}{P}=\left[1+\frac{\gamma-1}{2} M_{e}^{2}+Q_{c} K_{0, e}\right] G(\eta)-\frac{\gamma-1}{2} M_{e}{ }^{2} F^{2}(\eta)
$$

for $\eta \leq \eta_{f}$, and

$$
\frac{P_{e}}{P}=\left[1+\frac{\gamma-1}{2} M_{e}^{2}+Q_{e} K_{0, e}\right] G(\eta)-\frac{\gamma-1}{2} M_{e}^{2} F^{2}(\eta)-Q_{e} K(\eta)
$$

for $\eta \geq \eta_{f}$, where $Q_{e} \equiv f^{*} q /\left(c_{p} T_{e}\right)$. We note here that the heat capacity is assumed to be constant, similar to assumptions made by Prakash and Sirignano (1980) and Rangel and Femandez-Pello (1984). Calculations involving the variation of $c_{p}$ with temperature and gas composition will be presented in Chapter 5 .

The blowing velocity at the droplet surface, after the Levy-Lees transformation, gives

This gives in turn

$$
P_{B} v_{B}=-\frac{P_{e} \mu_{c} U_{e} r_{z}}{(2 \xi)^{1 / 2}} f(0) .
$$




$$
\frac{P_{B} v_{B}}{P_{\infty} u_{\infty}}=-\frac{3}{2}\left[\frac{2}{R e_{\infty}}\right]^{/ / 3}\left[\frac{\sin ^{4} \theta}{2-3 \cos \theta+\cos ^{3} \theta}\right]^{1 / 2} f(0) \text {. }
$$

so that

$$
\frac{v_{B}}{u_{\infty}}=O\left[\operatorname{Re}_{-\infty}^{-1 / 2} \frac{T_{B}}{T_{\infty}}\right] \leq O\left(\operatorname{Re}_{\infty}^{-1 / 2}\right) .
$$

This justifies the use of the boundary layer equations, for the error introduced by the radial velocity component is of the same order as the error of the boundary layer approximation itself.

The solution of the set of ordinary differential equations (2.22) is complicated by the fact that the tangential and normal (blowing) velocities, temperature and species mass fractions at the liquid surface are all unknown as yet. The tangential velocity $u_{5}(x)$ at the surface is obtained by coupling the gas-phase flow to the liquid-phase motion through shear-stress continuity. This yields for the unknown vortex strength $A$ in nondimensional form

$$
\frac{A R^{2}}{u_{\infty}}=\frac{3}{10} \frac{\mu_{\infty}}{\mu_{1}}\left[\frac{R e_{-\infty} \cos \theta}{\beta}\right]^{1 / 2} F^{\prime}(\eta=0)+O\left(M_{-}^{2}\right) .
$$

Obviously, eq. (2.25) gives different values for the vortex strength $A$ at different angular positions $\boldsymbol{\theta}$. A unique value for $A$ is obtained by approximating it by its value at the forward stagnation point, i.e.

$$
\frac{A R^{2}}{u_{\infty}}=\frac{3}{10} \frac{\mu_{\infty}}{\mu_{1}}\left(2 R e_{\infty}\right)^{1 / 2} F^{\prime}(\eta=0)+O\left(M_{\infty}^{2}\right) .
$$

(We note that approximating the vortex strength by its value by some other angular position, e.g. by its average value for $0 \leq \theta \leq \pi / 2$ would change the obtained value by a factor of 0.83 only.) The surface velocity is then obtained as $u_{b}=A R^{2} \sin \theta$ from (2.10). The mass blowing rate $p_{s} v_{b}(x)$ at the surface is determined using the 
assumption of negligible heat flux into the liquid phase at the interface. This condition implies that $p_{s} v_{s} h_{y}=\left[\lambda \frac{\partial T}{\partial y}\right]_{B}$, which in nondimensional form gives

$$
f(0)=-\frac{c_{p} T_{e}}{P_{t} h_{v}}\left[\left(1+\frac{\gamma-1}{2} M_{e}^{2}+Q_{e} K_{0, e}\right) G^{\prime}(0)-(\gamma-1) M_{e}^{2} F(0) F^{\prime}(0)\right] \text {. }
$$

The latent heat of vaporization, $h_{v}$ is assumed constant along the liquid interface, an assumption which is justified by an approximate calculation with variable $h_{v}$. Two additional relations are required to determine the fuel mass fraction and the temperature at the surface, and these are provided by the condition that the net flux of combustion products through the liquid interface must be zero, and by the Antoine equation for equilibrium evaporation. The former implies that

$$
P_{s} v_{s}\left(1-K_{f, s}\right)+P_{s} D_{s}\left(\frac{\partial K_{f}}{\partial y}\right]_{s}=0
$$

which, recalling the definitions of $K(\eta)$ and $f(\eta)$, reduces to

$$
K(0)=-\frac{1}{f}-\frac{1}{\operatorname{Pr}} \frac{K^{\prime}(0)}{f(0)}
$$

The partial pressure of fuel vapor at surface, $\mathrm{P}_{\mathrm{f}, \mathrm{s}}$, is related to the mass fraction of fuel vapor, $K_{f, s}$ through the molecular weights of the species:

$$
P_{f_{s} s}=P_{e} \frac{K_{f, s} / W_{f}}{K_{f, s} / W_{f}+\left(1-K_{f, s}\right) / W_{P}}
$$

Partial fuel vapor pressure, in turn, is related to the surface temperature $T_{8}$ through an equilibrium evaporation relation, e.g. the Antoine relation,

$$
T_{s}=\frac{B}{A-\log _{10} P_{1.8}}-C \text {, }
$$


where $\mathrm{T}_{8}$ and $\mathrm{Pf}_{\mathrm{f}, \mathrm{s}}$ are given in ${ }^{\circ} \mathrm{C}$ and $\mathrm{mm} \mathrm{Hg}$, respectively, and the constant coefficients A, B and C can be found in tables, such as Rossini et al. (1953) for hydrocarbon fuels. As an alternative to the Antoine relation, the Langmuir-Knudsen relation for kinetic equilibrium has also been employed, as done by Bellan and Summerfield (1978), with very little observed difference in the evaporation rates. In order to be consistent with the assumption that the effects of the liquid-phase viscous layer are negligible, i.e. the surface contour $r=R$ is a streamline, a constant temperature is assumed everywhere on the droplet surface. (The rationale for assuming a constant temperature along a streamline is given in the context of transient liquid phase heating in Chapter 3.) Calculations with a variable temperature along the surface show that the change in surface temperature is minimal, which justifies this assumption.

The set of coupled ordinary differential equations (2.22) is solved numerically by central differencing after quasi-linearizing the momentum equation (2.22a). The linearization of the quadratic term is

$$
F^{2}(\eta)=2 F^{*}(\eta) F(\eta)-\left[F^{*}(\eta)\right]^{2}+O\left[\left(F-F^{*}\right)^{2}\right] .
$$

where the superscript * indicates previous iteration values. The solution of the o.d.e.'s is carried out at discrete angular positions along the droplet surface until boundary layer separation is reached. Pseudo time-dependent calculations are performed by updating the Reynolds number through the calculated values of evaporation rate and drag at each time step. The dimensionless time scale $\tau$ used in these calculations is based on the free stream gas properies and the initial droplet radius, i.e., $\tau=\alpha_{\infty} t / R_{0}^{2}$. Here $\alpha_{\infty}$ denotes the thermal diffusivity of the freestream gas. Time steps $\Delta \tau$ are of the order of unity. 


\subsubsection{Calculation of Drag and Evaporation Rate}

Both pressure and shear stress at the surface contribute to the drag force $F_{D}$ exerted on the droplet by the convective stream. The drag coefficient $C_{D} \equiv 2 F_{D} /\left(\pi R^{2} P_{\infty} u_{\infty}^{2}\right)$ is thus computed from the pressure distribution determined from the external flow solution and from the shear stress distribution determined from the boundary layer solution. Since the boundary layer model described in this section does not allow for calculations beyond the separation point $\theta_{3}$, average quantities are employed to account for the pressure contributions in the region $\theta_{8} \leq \theta \leq \pi$. $\theta_{8}$ is determined as the angular position of zero shear. Both measurements and numerical calculations of the pressure distribution on the surface of a sphere in the Reynolds number range of interest, as outlined by Clift et al. (1978), indicate that a constant pressure at half that of the separation point value is a reasonable approximation for the pressure distribution in the region between the separation point and the rearward stagnation point. Fig. 2-3 shows the pressure distribution along the droplet surface calculated by the present model for a rigid sphere without mass transfer at the surface, at two different Mach numbers. The variation of the pressure coefficient does not depend on the Reynolds number in this potential-flow solution for the external flowfield. Comparison is made in Fig. 2-3 with the result of a fully numerical calculation given by Clift et al. (1978) for $\mathrm{Re}_{-}=100$. Experimental distributions are also given by Clift et al. (1978), but these are for Reynolds numbers of $O\left(10^{5} \ldots 10^{6}\right)$, and therefore not included in the comparison.

Fig. 2-4 shows the variation of dimensionless vorticity at the surface of a rigid sphere, $\omega_{s} R / u_{\ldots}$, which is proportional to the surface shear stress, with the angulas distance $\theta$ from the forward stagnation point. The comparison with the fully numeri- 
cal results presented by Clift et al. (1978) shows that the main contribution to the friction drag comes from the region $0 \leq \theta_{s}$.

The prediction of separation deserves special attention here. The calculation of the pressure distribution at the surface from the potential-flow solution does not allow us to capture the Reynolds number dependence of separation. As seen in Fig. 2-4, for compressible flow over a rigid sphere, the present model predicts separation at $\theta=97^{\circ}$. In reality, however, separation does depend on $R e_{\ldots}$. For instance, the fully numerical calculation given by Clift et al. (1978), and plotted in Fig. 2-4, predicts separation at $\theta=120^{\circ}$ for a $R e_{\infty}=100$-fiow over a rigid sphere - in good agreement with experimental observations. For higher Reynolds numbers, separation is closer to droplet shoulder. In terms of friction drag, however, the contribution of the region near, and beyond, the separation point bears much less weight than the region $0 \leq \theta \leq \frac{\pi}{2}$. Thus, for the calculation of drag in the present model, the surface shear stress beyond the predicted separation angle is neglected with respect to its contribution to total drag. For a rigid sphere, this approximation results in the correlation

$$
C_{D}=0.4926+6.1005 \operatorname{Re}_{\infty}^{-1 / 2}+O\left(M_{\infty}^{2}\right)
$$

for the drag coefficient. In eq. (2.31), the first term on the right hand side represents the pressure drag which is, as mentioned above, independent of $\operatorname{Re}_{\infty}$. The second term is the friction drag, with the $\mathrm{Re}_{\infty}^{-1 / 2}$-dependence typical of the laminar boundary layer approximation.

The curve described by eq. (2.31) is plotted in Fig. 2-5, along with the "Standard Drag Curve (Clift et al. (1978)", and the Stokes and Oseen approximations for low Reynolds number flows. In the Reynola's number regime of inierest in this 
study, $50 \leq R_{e} \leq 200$, our prediction is relatively close to the standard drag curve. The error is about 13 percent for $\mathrm{Re}_{\infty}=50$, about 19 percent for $R e_{-}=200$, and remains under 1 percent for $80 \leq$ Re $_{\infty} \leq 110$. Finally, Clift et al. (1978) show that, in terms of both skin friction and surface pressure, the values for a rigid sphere are very close to those for a fluid sphere (e.g. water drop in air) for $R e_{\infty}=O(100)$.

The total mass transfer, i.e., the rate of evaporation from the droplet, $\dot{m}$, is given by

$$
\dot{\mathrm{m}}=\int_{0}^{\pi}\left(p_{s} v_{s}\right)\left(2 \pi R^{2} \sin \theta d \theta\right)
$$

For the present set of calculations, the contribution of the region beyond the separation point to the overall evaporation rate is neglected. As noted by Prakash and Sirignano (1980), and as will be shown below, the rate of mass transfer in the region of the flow beyond the separation point is relatively small. The results presented here can thus be regarded as a lower estimate in terms of convective droplet evaporation. Numerical results presented by Clift el al. (1978) for mass transfer from spheres also justify this assumption at low and moderate Reynolds numbers, i.e. $\operatorname{Re}_{\infty} \leq \mathrm{O}(100)$.

\subsection{Results and Discussion}

In the analysis of Harper and Moore (1968), the velocity at the surface is taken to be only a small perturbation of its value at the edge of the viscous liquid layer. Prakash and Sirignano (1978) show that this is a valid assumption while the same may not be true for vorticity, since the difference in vorticity between the surface and the edge of the viscous layer may not be negligible. However, as seen in Fig. 2 - 
6, the effect of liquid motion on the profiles in the gaseous boundary layer, and consequently on the transfer processes at the surface, is relatively small, as also observed by Rangel and Femandez-Pello (1984). Thus, as mentioned in Section 2.1 2. the surface motion is approximated by the inviscid inner flow solution at the limit $\mathbf{r}=\mathbf{R}$, which reduces the required computational time for the solution of the coupled gas-liquid boundary layers considerably, without causing any significant effect on drag and evaporation rate predictions.

For the case of the burning droplet, where the existence of an envelope flame is assumed a priori, the position of the flame before separation can be calculared. The experimental results of Gollahalli and Brzustowski (1972) and Teodorczyk and Wojcicki (1983) indicate that the assumption of an envelope flame can be considered realistic for $R e_{\infty}<200$, although the recent results of Gokalp et al. (1988) show that transition to a wake flame may occur at lower Reynolds numbers in microgravity.

Calculated temperature, velocity, and species concentration profiles in the boundary layer for specific freestream conditions are shown in Fig. 2-6. The profiles in this figure indicate that the influence of the internal flowfield of the droplet, of the order of 5 percent of the flow magnitude at the boundary layer edge, is small. While the profiles shown in Fig. 2-6 correspond to a point $60^{\circ}$ from the stagnation point of ine droplet, similar behavior is noted at other locations along the droplet surface.

The equilibrium determination of the liquid surface temperature in our analysis is seen also to affect mass loss from the surface, as indicated in Fig. 2-7. Comparison is riade here with the predictions of Rangel and Femandez-Pello (1984), who assume a constant surface (and liquid) temperature, that of the boiling point for the given freestream pressure. Thus, these researchers obtain a substantial increase in evaporative blowing near the stagnation point, and a prediction of boundary-layer 
separation that lies upstream of the present calculation.

It is also noted that the effect of Mach number on droplet evaporative and burning processes is not strong; this is indicated in Fig. 2-8. As is physically reasonable, the local shear stress near the top of the droplet surface is slightly increased with higher freestream Mach number, but this effect is negligible closer to the stagnation point. The effect of Mach number on mass loss appears to be negligible at all locations along the droplet, although in the absence of the flame (evaporation only), the increased temperature and pressure at the stagnation point with higher $M_{\infty}$ does slightly increase the mass transfer in that region. Fig. $2-8$ also indicates, as noted above, that as the point of separation is approached, the local mass transfer rate becomes very small.

The computed variation in drag coefficient for evaporating and buming droplets is shown in Fig. 2-9, which also provides comparison with the experimental results of Yuen and Chen (1976) for an evaporating droplet, and with the "standard curve" for the drag coefficient of a solid sphere (Clift et al. (1978)). The present calculations appear to correspond well to experimental observations and indicate the significant influence of mass blowing on the behavior of the drag coefficient. The calculated rate of mass loss as a function of Reynolds number is shown in Fig. 2-10. for both evaporating and burming droplets at different freestream conditions. Direct comparison is made with a Froessling/Ranz-Marshall type of correlation (see eqs. (1.4) and (1.7)) as well as the experimental data of Renksizbulut and Yuen (1983a), eq. (1.11) for the evaporation rate from a suspended droplet in a hot stream. It is noted here that most other experimental observations on evaporating and buming droplets in a convective environment always involve highly scattered data. In this context, the measurements of Eisenklam, et al. (1966) and Natarajan and 
Brzustowski (1970) can also be referenced, although the average of the empirical correlations given in both of these papers result in evaporation rates that are somewhat higher that those reported by Renksizbulut and Yuen (1983a) as well as those calculated presently. As noted by Sanders and Dwyer (1987), this discrepancy could be due to differences in specific mechanisms present (perhaps initial transient droplet heating or evaporation in the wake region) which affect the mass transfer.

Figure 2-11 displays results for the variation in droplet radius and Reynolds number with dimensionless time, $\tau$, including comparison with the experimental observations of Renksizbulut and Yuen (1983a). The initial Reynolds number is chosen to be 200 , and the initial droplet radius $1 \mathrm{~mm}$, for correspondence to the experiments. The drag coefficient used to extrapolate the experimental values is taken from the data of Yuen and Chen (1976). Finally, predicted flame shapes, based on local maxima in boundary layer temperature (and the local vanishing of the reactant mass fractions) are shown in Fig. 2-12 for two different Reynolds numbers. It should be noted that the fiame shapes shown are each relative to the instantaneous droplet radius $\mathbf{R}(\tau)$. Separation of the flame from the region near the droplet surface appears to coincide with separation of the extemal boundary layer.

\subsection{Summary}

The results presented in this chapter demonstrate that, by including only the crucial physical phenomena associated with fuel droplet vaporization and buming in a convective environment, a very reasonable representation for droplet behavior can be obtained. By using analytical representations of the inviscid gas and liquid internal flowfields, and by employing the boundary layer type of assumptions first used 
by Prakash and Sirignano (1980) and later by Rangel and Femandez-Pello (1984), it is possible to solve for the characteristics of the reacting flow adjacent to the droplet surface. These characteristics allow calculation of parameters which describe droplet evaporation and burning (drag coefficient, mass transfer rates, size histories) that compare very well with the experimental data of Renksizbulut and Yuen (1983a) and Yuen and Chen (1976). As noted above, there is a relatively large degree of scatter present in the experimental data available for burning droplets, e.g., scatter over nearly an order of magnitude in the measured mass loss rates (see Natarajan and Brzustowski (1970)). Hence, comparison of the burning rates with the present model's results becomes less precise. It should be noted, however, that the current predictions lie well within the error bars of the experimental data, but tend slightly to underpredict (compared with the average of the data) the degree of mass loss by the droplet. Current predictions for mass loss are also somewhat lower than the fullscale numerical predictions made by Dwyer and Sanders (1986), but this may be due to the fact that these researchers choose to linearize the exponential term in the equilibrium relation used. Despite these minor discrepancies, our model appears to be relatively robust and accurate, with a minimum in required computational times (on the order of 30 seconds on an IBM 3090-600S), and thus allow's detailed parametric studies to be performed with ease. 


\section{CHAPTER 3 TRANSIENT EVAPORATION AND BURNING}

\subsection{Transient Heating of the Droplet Interior}

The case considered in Chapter 2 was the quasi-steady evaporation and buming process of a fuel drop in a convective environment. It is relevant for practical combustor situations only when the drop has reached a nearly uniform temperature since the heat-up process of the drop cannot be considered quasi-steady. As already mentioned in Chapter 1, especially for heavy fuels at high freestream pressures and temperatures, the heat-up period can be as long as the drop lifetime itself for small drops, say $10^{-2} \mathrm{~mm} \leq R_{0} \leq 1 \mathrm{~mm}$. This was pointed out also by Prakash and Sirignano (1978). To account for the entire lifetime of the droplet, the transient heating process of the droplet interior has to be considered.

\subsubsection{Streamline Coordinates}

The unsteady energy equation for a liquid with constant thermal diffusivity $\alpha_{1}$ is

$$
\frac{\partial T_{1}}{\partial t}+u \cdot \nabla T_{1}=\alpha_{1} \nabla^{2} T_{1}
$$


where $T_{1}$ is the liquid temperature, $u$ is the velocity vector, $\nabla$ stands for gradient and $\nabla^{2}$ for the Laplace operator.

Now we can write equation (3.1) in terms of the orthogonal streamline coordinate system $(\xi, \psi, \omega)$, as shown in Fig. 3-1. The streamsurfaces are given by equation (2.9). We let $\psi_{0}=A R^{2} / 8$, so that at the vortex core, i.e. $2 \mathrm{r}=(1 / 2)^{k / 2}, \theta=\pi / 2$, the stream function $\psi$ has the value 0 . Then the stream function $\psi$ is

$$
\Psi=\frac{1}{8} A R^{4}\left[1-4 \hat{r}^{2}\left(1-\hat{r}^{2}\right) \sin ^{2} \theta\right] .
$$

The velocity components are

$$
\begin{aligned}
& u_{r}=\frac{1}{r^{2} \sin \theta} \frac{\partial \psi}{\partial \theta}=-A R^{2}\left(1-\hat{r}^{2}\right) \cos \theta, \\
& u_{\theta}=-\frac{1}{r \sin \theta} \frac{\partial \psi}{\partial r}=A R^{2}\left(1-2 \hat{r}^{2}\right) \sin \theta .
\end{aligned}
$$

We note that the velocity vectors are always along streamlines, i.e.

$$
u=|u| e_{\xi}=\left[u_{s}^{2}+u\right]_{\xi}^{2 / 2} e_{\xi} \text {. }
$$

where $e_{\xi}$ is the unit vector in the $\xi$-direction. Thus the streamwise velocity $u_{\xi}$ can be written as

$$
u_{\xi}(\hat{r}, \theta)=A^{2}\left[\left(1-\hat{r}^{2}\right)^{2} \cos ^{2} \theta+\left(1-2 \hat{r}^{2}\right)^{2} \sin ^{2} \theta\right]^{1 / 2}
$$

The differential operators in the orthogonal curvilinear coordinate system $(\xi, \psi, \omega)$ are (see e.g. Batchelor (1967))

$$
\nabla=\frac{1}{h_{\xi}} \frac{\partial}{\partial \xi} e_{\xi}+\frac{1}{h_{\psi}} \frac{\partial}{\partial \psi} e_{\psi}+\frac{1}{h_{\omega}} \frac{\partial}{\partial \omega} e_{\omega v}
$$




$$
\nabla^{2}=\frac{1}{h_{\xi} h_{\psi} h_{\omega}}\left[\frac{\partial}{\partial \xi}\left[\frac{h_{\psi} h_{\omega}}{h_{\xi}} \frac{\partial}{\partial \xi}\right]+\frac{\partial}{\partial \psi}\left[\frac{h_{\omega} h_{\xi}}{h_{\psi}} \frac{\partial}{\partial \psi}\right]+\frac{\partial}{\partial \omega}\left[\frac{h_{\xi} h_{\psi}}{h_{\omega}} \frac{\partial}{\partial \omega}\right)\right]
$$

where the metric scale factors are given by (see Kronig and Brink (1951))

$$
\begin{gathered}
h_{\xi}=\frac{A R}{8} \frac{\left(2 \hat{r}^{2}-1\right)^{2}}{u_{\xi} \hat{r}^{3} \cos ^{3} \theta} . \\
h_{\psi}=\frac{1}{R u_{\xi} \hat{r} \sin \theta} . \\
h_{\omega}=R \hat{r} \sin \theta .
\end{gathered}
$$

Lines orthogonal to the streamlines are then given by

$$
\xi=2 R^{2} \frac{\hat{r}^{4} \cos ^{4} \theta}{1-2 \hat{r}^{2}}=\text { const. }
$$

Substituting (3.6a) and (3.6b) in the energy equation, considering that from (3.4)

$$
u \cdot \nabla T_{1}=\frac{u_{\xi}}{h_{\xi}} \frac{\partial T_{1}}{\partial \xi} .
$$

and accounting for axial symmetry $(\partial / \partial \omega=0)$, we obtain

$$
\frac{\partial T_{1}}{\partial t}+\frac{u_{\xi}}{h_{\xi}} \frac{\partial T_{1}}{\partial \xi}=\frac{a_{1}}{h_{\xi} h_{\psi} h_{\omega}}\left[\frac{\partial}{\partial \xi}\left[\frac{h_{\psi} h_{\omega}}{h_{\xi}} \frac{\partial T_{1}}{\partial \xi}\right]+\frac{\partial}{\partial \psi}\left[\frac{h_{\omega} h_{\xi}}{h_{\psi}} \frac{\partial T_{1}}{\partial \psi}\right]\right] .
$$

As argued by Kronig and Brink (1951) and Pan and Acrivos (1968), the time for a liquid particle to travel once along a closed streamline is much shorer than the characteristic time of heat diffusion for high Peclet number circulation. This means that at any time, the average temperature for a streamsurface will be a valid approximation for the temperature at any point on the same streamsurface. As mentioned in Chapter 1, Pan and Acrivos (1968) show that this is a valid assumption for large liquid Peclet numbers, the error in this approximation being $\mathrm{O}\left(\mathrm{Pe}_{i}^{-1}\right)$, or, 
$O\left(\operatorname{Pr}_{1}^{-1} \operatorname{Re}_{1}^{-1}\right)$. For the cases considered here, the liquid Reynolds number $\operatorname{Re}_{1}$, defined as $R e_{1}=p_{1}\left(A R^{2}\right)(2 R) / \mu_{1}$, is $O\left(R e_{-}\right)$and liquid Prandl numbers for hydrocarbon fuels are typically $\mathrm{O}(10)$, so that $\mathrm{Pe} \mathrm{C}_{1}=\mathrm{O}\left(10^{3}\right)$.

The average temperature on a streamline, $\overline{\mathrm{T}}_{1}(\psi, t)$, is given by

$$
\bar{T}_{1}(\psi, t)=\frac{\int T_{1} h_{\xi} h_{\psi} h_{\omega} d \xi}{\int h_{\xi} h_{\psi} h_{\omega} d \xi} .
$$

Then,

$$
\frac{\partial \bar{T}_{1}}{\partial \psi}=\frac{\int \frac{\partial T_{1}}{\partial \psi} \frac{h_{\omega} h_{\xi}}{h_{\psi}} d \xi}{\int \frac{h_{\omega} h_{\xi}}{h_{\psi}} d \xi} .
$$

Integrated along a closed streamline, the energy equation (3.9) reduces to

$$
\frac{\partial}{\partial t} \int T_{1} h_{\xi} h_{\psi} h_{\omega} d \xi=\alpha_{1} \frac{\partial}{\partial \psi} \int \frac{h_{\omega} h_{\xi}}{h_{\psi}} \frac{\partial T_{1}}{\partial \psi} d \xi
$$

since $\left(u_{\xi} / h_{\xi}\right)\left(h_{\xi} h_{\psi} h_{\omega}\right)=1$ and $\int\left(\partial T_{1} / \partial \xi\right) d \xi=0$.

Using the definition of the average temperature $\bar{T}_{1}(\psi, 1)$, eqs. (3.10) and (3.10a), we obtain for steady flow, i.e. $\int h_{\xi} h_{\psi} h_{\omega} d \xi=0$,

$$
\frac{\partial \bar{T}_{1}}{\partial t}=\frac{A^{2} R^{6}}{64} \frac{\alpha_{1}}{J_{1}(\psi)} \frac{\partial}{\partial \psi}\left[J_{2}(\psi) \frac{\partial \bar{T}_{1}}{\partial \psi}\right] .
$$

where

$$
\begin{aligned}
& J_{1}(\psi) \equiv \frac{A R}{8} \int h_{\xi} h_{\psi} h_{\omega} d \xi, \\
& J_{2}(\psi) \equiv \frac{8}{A R^{j}} \int \frac{h_{\omega} h_{\xi}}{h_{\psi}} d \xi .
\end{aligned}
$$

The values of the functions $J_{1}(\psi)$ and $J_{2}(\psi)$ within the range of interest, i.e. 
$0 \leq \psi \leq \psi_{0}$, are shown in Fig. 3-2.

Finally, introducing the nondimensional variables $\tau_{1}=\alpha_{1} t / R^{2}$ and $\hat{\psi} \equiv 8 \psi /\left(A R^{4}\right)$, equation (3.12) can be written as

$$
\frac{\partial \bar{T}_{1}}{\partial \tau_{1}}=\frac{1}{J_{1}(\hat{\psi})} \frac{\partial}{\partial \hat{\psi}}\left[J_{2}(\hat{\psi}) \frac{\partial \bar{T}_{1}}{\partial \hat{\psi}}\right] .
$$

\subsubsection{Initial and Boundary Conditions}

At time $t=0$ we assume that the drop will be at a uniform temperature $T_{0}$. This corresponds to the physical situation of a cold drop being exposed to a hot environment, thus heating up and evaporating simultaneously. This condition can be written as

$$
\bar{T}_{1}(\hat{\psi})=T_{0} \quad \text { at } \tau_{1}=0
$$

The differential equation (3.14) will be valid at the vortex core, i.e. at $\hat{\psi}=0$, as at any other value of $\hat{\psi}$. Noting that $J_{2}(\hat{\psi}=0)=0$ and the second derivative of $\bar{T}_{1}$ with respect to $\hat{\psi}$ being finite at $\hat{\psi}=0$, however, it reduces to

$$
\frac{\partial \bar{T}_{1}}{\partial \tau_{1}}=\frac{1}{J_{1}} \frac{\partial J_{2}}{\partial \hat{\psi}} \frac{\partial \bar{T}_{1}}{\partial \dot{\psi}} \quad \text { at } \hat{\psi}=0
$$

At the surface, the temperature gradient is determined by the amount of heat transfer from the gas into the liquid phase, $\dot{Q}$, which is obtained by integration over the surface area of the drop:

$$
\dot{Q_{1}}=\int_{0}^{\pi}\left[-\lambda_{1} \frac{\partial \bar{T}_{1}}{\partial \tau}\right]_{3} 2 \pi R^{2} \sin \theta \text {. }
$$


where $\lambda_{1}$ is the thermal conductivity of the liquid, and from (3.2)

$$
\left[-\lambda_{1} \frac{\partial \bar{T}_{1}}{\partial r}\right]_{2}=-\lambda_{1}\left[\frac{\partial \bar{T}_{1}}{\partial \psi}\right]_{i=1} 8 \sin ^{2} \theta \text {. }
$$

This yields, formulated as a boundary condition,

$$
\frac{\partial \bar{T}_{1}}{\partial \dot{\psi}}=-\frac{3}{64 \pi} \frac{\dot{Q_{1}}}{\lambda_{1} R} \quad \text { at } \dot{\psi}=1 \text {. }
$$

\subsection{Coupling of Gas and Liquid Phase Solutions}

Now, the equation (3.12), combined with the initial and boundary conditions (3.15), (3.16) and (3.18) can be solved numerically, e.g. by finite differencing. The surface heat transfer $\dot{Q}_{1}$ is unknown, and therefore, the solution of the transient liquid phase heating must be coupled with the gas-phase boundary layer solution. This coupling is given by the heat-transfer boundary condition for the gaseous boundary layer

$$
\dot{Q}_{B}=\dot{m} h_{v}+\dot{Q}
$$

Then, the "effective" latent heat of vaporization, defined as $h_{v, e f f}=\dot{Q}_{B} / \dot{m}$, is related to the liquid heating rate through the relation

$$
h_{v, \text { eff }}=h_{v}+\frac{\dot{Q}}{\dot{m}} .
$$

We note that the present solution approaches the steady-state solution, described in Chapter 2, as $\dot{\mathbf{Q}} \rightarrow \mathbf{0}$, or, $h_{v} \rightarrow h_{v, e \pi}$. This is the case for the later part of the droplet lifetime when the liquid iemperature becomes nearly uniform and its gradient vanishes. In other words, the steady-state solution is the solution for the 
same problem for the special case $\dot{Q}_{\mathbf{A}}=0$, or $h_{v}=h_{v, e f f}$, which simplifies the boundary condition (3.19) for the gaseous boundary layer problem.

The iterative solution of the coupled problem involves a first guess for the surface temperature $T_{3}=\bar{T}_{1}(\hat{\psi}=1)$ at each time step. Then the gaseous boundary layer is solved iteratively as in the steady-state calculation. This time, however, we have $\dot{\mathbf{Q}}$, or $h_{\mathrm{v}, e f f}$, as an additional unknown to be solved for, and the transient liquid-phase energy equation formulated in this chapter provides an additional relation. Gas-

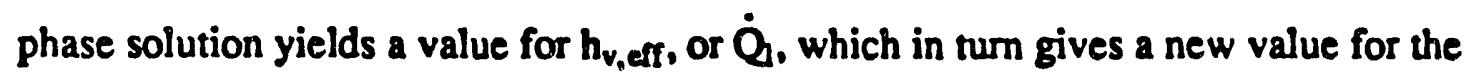
surface temperature $\bar{T}_{1}(\hat{\psi}=1)=T_{3}$. This procedure is carried out at each time step until the solution for $T_{s}$ converges.

\subsection{Results and Discussion}

\subsubsection{Errective Transfer Number}

The variation of the droplet size and drag is calculated using the same methodology as in the steady-state case, which is outlined in Chapter 2. Comparison of the results is made with the empirical correlations of Froessling/Ranz-Marshall, Renksizbulut-Yuen and Natarajan-Brzustowski, given by eqs. (1.4), (1.11) and (1.10), respectively. It is observed that all of these correlations involve the transfer number B. In the literature (see e.g. Kuo (1986)) B is often defined as a mass transfer number, $B_{m}$.

$$
B_{m}=\frac{K_{f, s}-K_{f_{100}}}{1-K_{I_{15}}}
$$

as in eq. (1.6), but also as a steady-state heat transfer number, 


$$
B_{L_{2, S B}}=\frac{C_{p}\left(T_{-}-T_{B}\right)}{h_{v}}
$$

It is interesting to note that for the special case of unity Lewis number, i.e. $\mathrm{Pr}=\mathrm{Sc}$, and if the droplet has uniform temperature, i.e. $\dot{Q}=0$, these two numbers are the same:

$$
B_{m}=B_{L, 2 s}=B \quad \text { for } L e=1 \text { and } \dot{Q}_{1}=0 \text {. }
$$

This was the case for the steady-state calculations of Chapter 2. However, now that we are also considering the initial, transient part of the droplet lifetime, i.e. the heatup period when $\dot{Q}_{1} \neq 0$ and consequently, from (3.20), $h_{v, e f l} \neq h_{v}$, the thermal transfer number has to be modified to represent the analogy between the heat and mass transfer appropriately. This is achieved by letting

$$
B_{\mathrm{Lh}}=\frac{C_{\mathrm{p}}\left(\mathrm{T}_{-}-\mathrm{T}_{\mathrm{B}}\right)}{h_{\mathrm{v}, \text { eff }}}
$$

It can be seen easily from eqs. (3.22) and (3.24) that as the droplet heat-up is being completed and as the process is becoming steady-state, i.e. $h_{v, \text { eff }} \rightarrow h_{v}, B_{u_{h}} \rightarrow B_{u_{h}, s s}$. In other words, by defining the thermal transfer number as in eq. (3.24), $B_{m}=B_{t h}=B$ holds for the entire drop lifetime provided that $L e=1$.

\subsubsection{Comparison with Empirical Correlations}

The effective transfer number given by eq. (3.24) is used in the correlations (1.4), (1.11) and (1.10) when comparison is made between the predictions of the present model and these correlations. The result is summarized in Fig. 3-3. It is very interesting to note that the agreement of the present model with Froessling/RanzMarshall and Renksizbulut-Yuen type of correlations is fairly good not only for the 
evaporation case but also for the buming drop. This is significant because these correlations were developed for the steady-state, non-reacting evaporation rates from drops. Modifying the transfer number $B$ as in eq. (3.24) to account for unsteady heating, we seem to have shown that this type of a correlation can be a fairly good predictor for the convective evaporation behavior of a drop in the presence as well as in the absence of an invelope flame surrounding it.

As for the correlation of Natarajan and Brzustowski, the observation is made that the values obtained from this correlation lie higher than our predictions by about a factor of two, as in the steady-state calculations (see Chapter 2). As mentioned in Chapter 2, however, we still are within the error margin of this correlation considering the scatter of Natarajan and Brzustowski's (1972) data over an entire order of magnitude.

The case considered for these calculations was chosen thus as to enable a direct comparison with the transient model of Prakash and Sirignano (1980). The fuel is $n$ Decane, the freestream pressure and temperature are $10 \mathrm{~atm}$ and $1000 \mathrm{~K}$, respectively. The time-dependent calculation is started with the initial values $\operatorname{Re}_{-, 0}=200$, $R_{0}=50 \mu \mathrm{m}$ and $T_{0}=300 \mathrm{~K}, T_{0}$ being the uniform initial temperature of the drop. In order to repeat the case calculated by Prakash and Sirignano, the relative velocity was taken to be constant throughout this calculation. In other words, no deceleration was considered due to drag. Figure 3-4 shows the predicted temporal change in droplet radius in comparison with the previous predictions of Prakash and Sirignano (1980). The latter slightly overpredict the evaporation rate and, consequently, underpredict the lifetime of the drop for the case without chemical reaction (considering the results in Fig. 3-3). Predictions for a buming drop are also displayed in Fig. 3-4, but a direct comparison with Prakash and Sirignano (1980) is not possible 
in this case since they have not reported calculations for burning drops. The predicted surface temperatures are compared in Fig. 3-5, and good agreement is observed. However, the results shown for Prakash and Sirignano (1980) represent the variation of the surface temperature at drop shoulder $\left(\theta=90^{\circ}\right)$, whereas we assume a constant temperature along the drop surface and approximate it by its value at the forward stagnation point $\left(\theta=0^{\circ}\right)$. It is clear that the temperature at the forward stegnation point will be higher than that at the shoulder, but this means that Prakash and Sirignano are essentially predicting a higher surface temperature, and, consequently, a higher evaporation rate. The discrepancy for the predicted surface temperature between the two models is most probably caused by the difference in the relations used to represent equilibrium evaporation at drop surface.

A second sample calculation is carried out in order to compare the present model's predictions with those obtained by Sanders and Dwyer (1987) through their fully-numerical model. The fuel for this case is $n$-Decane, the freestream pressure and temperature are $5 \mathrm{~atm}$ and $1000 \mathrm{~K}$, respectively. The initial values are $R e_{\infty, 0}=100, R_{0}=50 \mu \mathrm{m}$ and $T_{0}=400 \mathrm{~K}$. This time the deceleration of the drop due to drag was taken into account as was done by Sanders and Dwyer (1987). Again, apparently due to different relations used for equilibrium evaporation at the drop surface there is a discrepancy between the two predictions for the history of an evaporating drop, as shown in Fig. 3-6. As already mentioned in Chapter 2, in the present study the Antoine relation is used to represent equilibrium evaporation at the droplet surface, while Sanders and Dwyer (1987) have employed a linearized version of the Clausius-Clapeyron relation in their model. Sanders and Dwyer (1987) repor results for the burning case only for a drop with uniform temperature, that temperature being its boiling point value. As expected, their results for that case are higher 
than ours, and are likely to be higher than experimental observations (see Fig. 3-2). These results are not included in Fig. 3-6 since they are essentially for steady state. Compared with computational times of $O(1$ hour) on a Cray computer for fully numerical models (Dwyer (1989)), our computing times of O(1 min.) on IBM 3090 6005 for typical droplet history calculations as presented in this chapter demonstrate the nerits of the present analytical/numerical model in terms of reasonably predicting transient droplet burning behavior with a minimum in computing times.

\subsection{Summary}

In this chapter, a simplified model for the transient heating of the droplet interior, along the lines of the previous work by Kronig and Brink (1951) and Prakash and Sirignano (1978), is developed. This enables a coupled solution for the gas- and liquid-phase transfer processes, that is, simultaneous heating and evaporation or burning of a droplet in a convective flowfield.

The most significant result of these calculations is that, if an appropriate transfer number $B$ is used in Froessling/Ranz-Marshall type of correlations, these furn out to be fairly adequate predictors of transient evaporation rates from droplets not only for the non-reacting case but also for the case of an envelope flame existing around the droplet.

A comparison with the predictions of Prakash and Sirignano (1980) for the non-reacting case results in slight disagreement in terms of the evaporation rates, probably caused by the fact that these authors use a different relation to describe equilibrium evaporation at the droplet surface. The discrepancy in evaporation rates is more significant in the comparison with the fully numerical predictions of Sanders 
and Dwyer (1987), again for the non-reacting case. We note, however, that these authors use a linearized version of the Clausius-Clapeyron equation for equilibrium evaporation.

Finally, in terms of computing times, the present model compares very favorably with fully numerical models such as that of Dwyer and Sanders (Dwyer (1989)) - considering that this simplified model enables a very reasonable prediction of transient droplet burning. 


\section{CHAPTER 4 \\ SINGLE-STEP, FINITE-RATE CHEMISTRY}

So far in this study we have chosen to represent the burning process with infinitely fast chemical reaction kinetics, which simplifies the goveming gaseous boundary layer equations considerably through the use of composite variables as described in Chapter 2. This type of approximation is useful for representing quasisteady burning processes, but it cannot be used to examine phenomena such as ignition and extinction of flames. For this purpose a finite-rate chemical kinetics model is necessary. As a first step in that direction, the boundary layer model described in the previous chapters is now extended as to account for a single-step, finite-rate chemical reaction.

\subsection{Chenistry}

For the general chemical reaction of the type

$$
[\text { Fuel }]+\alpha\left[\mathrm{O}_{2}\right] \rightarrow \text { Reaction products, }
$$

the molar rate of consumption of the fuel per unit volume is given by

$$
\frac{d C_{t}}{d t}=\frac{1}{\alpha} \frac{d C_{0}}{d t}=-k(T) C_{1} C_{0} .
$$


where $k$ is the chemical reaction rate, and $C_{f}$ and $C_{0}$ denote the molar concentration of fuel vapor and oxidizer in the gas mixture, respectively.

The Arrhenius law provides an expression for $k$.

$$
k(T)=A_{t} \exp \left[-\frac{E_{e}}{R_{d} T}\right] .
$$

where $A_{x}$ is the "frequency factor," in general a function of the temperature. For the calculations in this chapter, however, $A_{7}$ will be assumed constant. In eq. (4.2), $E_{2}$ is the activation energy, and $R_{u}$ is the universal gas constant. Now the fuel and oxidizer mass production rates, given by definition as

$$
\dot{w}_{i}=w_{i} \frac{d C_{i}}{d t}, \quad i=f, 0 ;
$$

where $W_{i}$ denotes the molecular weight of the corresponding species, can be written as

$$
\dot{w}_{f}=-\frac{\rho^{2}}{W_{0}} A_{t} \exp \left[-\frac{E_{a}}{R_{u} T}\right] K_{0} K_{f}
$$

and

$$
\dot{w}_{0}=-\frac{\rho^{2}}{W^{*} W_{0}} A_{r} \exp \left[-\frac{E_{1}}{R_{y} T}\right] K_{f} K_{0} .
$$

respectively, where $\mathrm{f}^{*}=\mathrm{W}_{\mathrm{f}} /\left(\alpha \mathrm{W}_{0}\right)$ is the stoichiometric fucl-oxidizer ratio, already mentioned in Chapter 2. The negative signs in eqs. (4.4) indicate that fuel and oxidizer are consumed by the flame, not produced.

\subsection{Boundary Layer Equations}


The energy equation for the gaseous boundary layer, eq. (2.13), can also be written as (see Kuo (1986))

$\rho\left[v \frac{\partial T}{\partial x}+v \frac{\partial T}{\partial y}\right]=-\frac{P_{e} u_{e}}{C_{p}} \frac{d u_{e}}{d x} u+\frac{\partial}{\partial y}\left[\frac{\mu}{P_{r}} \frac{\partial T}{\partial y}\right]+\frac{\mu}{C_{p}}\left[\frac{\partial u}{\partial y}\right]^{2}+\frac{\mu}{S c} \frac{\partial T}{\partial y} \sum_{i=1}^{N} \frac{\partial K_{i}}{\partial y}-q \dot{w}_{f}$

where the index $i$ in the summation refers to fuel vapor and oxidizer. It is assumed that the specific heat $c_{p}$ and the mass diffusivity $D$ are constant, and have the same value for all the species involved.

Using the expressions (4.4a) and (4.4b) for fuel and oxidizer mass mass production rates, the species equations can now be written as

$$
\begin{aligned}
& p\left[u \frac{\partial K_{f}}{\partial x}+v \frac{\partial K_{f}}{\partial y}\right]=\frac{\partial}{\partial y}\left[\frac{\mu}{S c} \frac{\partial K_{f}}{\partial y}\right]-\frac{p^{2} A_{t}}{W_{0}} \exp \left[-\frac{E_{a}}{R_{u} T}\right] K_{0} K_{f}, \\
& \rho\left[u \frac{\partial K_{0}}{\partial x}+v \frac{\partial K_{0}}{\partial y}\right]=\frac{\partial}{\partial y}\left[\frac{\mu}{S c} \frac{\partial K_{0}}{\partial y}\right]-\frac{p^{2} A_{t}}{W^{*} W_{0}} \exp \left[-\frac{E_{t}}{R_{u} T}\right] K_{f} K_{0} .
\end{aligned}
$$

The equations (2.11), (2.12), (4.5), (4.6) and (4.7) constitute the boundary layer equations for finite rate chemical reaction kinetics. These equations can be transformed to a set of ordinary differential equations using the Lees transformation outlined in Chapter 2. The resulting equations, assuming self-similar profiles for the velocity, temperature and species mass fractions in the boundary layer are

$$
\begin{aligned}
F^{\prime \prime}(\eta) & +f(\eta) F^{\prime}(\eta)+\beta(\xi)\left[\Theta(\eta)-F^{2}(\eta)\right]=0 . \\
\frac{1}{P_{\tau}} \Theta^{\prime \prime}(\eta)+[f(\eta) & \left.+\frac{1}{S c}\left(K_{f}^{\prime}(\eta)+K_{0}^{\prime}(\eta)\right)\right] \Theta^{\prime}(\eta)=-(\gamma-1) M_{e}^{2}\left[F^{\prime}(\eta)\right]^{2} \\
& -\frac{\Delta_{e} Q_{e}}{K_{l}(\eta) K_{0}(\eta) \frac{\exp [-E / \Theta(\eta)]}{\Theta(\eta)} .}
\end{aligned}
$$




$$
\begin{aligned}
& \frac{1}{S c} K_{f}^{\prime \prime}(\eta)+f(\eta) K_{f}^{\prime}(\eta)-\Delta_{e} K_{0}(\eta) \frac{\exp [-E / \Theta(\eta)]}{\Theta(\eta)} K_{f}(\eta)=0, \\
& \frac{1}{S c} K_{0}^{\prime \prime}(\eta)+f(\eta) K_{0}^{\prime}(\eta)-\frac{\Delta_{e}}{\gamma^{*}} K_{f}(\eta) \frac{\exp [-E / \Theta(\eta)]}{\Theta(\eta)} K_{0}(\eta)=0,
\end{aligned}
$$

where

$$
\theta(\eta)=\frac{T}{T_{e}}
$$

is a nondimensional temperature,

$$
E \equiv \frac{E_{e}}{R_{u} T_{c}}
$$

is a nondimensional activation energy, and

$$
\Delta_{\varepsilon} \equiv \frac{2 \xi A_{p}}{\mu_{c} u_{e}^{2} r_{s}^{2} W_{0}}
$$

The nondimensional parameter $\Delta_{c}$ can also be written as

$$
\Delta_{c}=\frac{2 D a_{\infty}}{S c R e_{\infty}} \frac{u_{\infty}}{\rho_{\infty} R} \frac{2 \xi}{\mu_{c} u_{e}^{2} r_{s}^{2}} \text {. }
$$

where

$$
D_{a}=\frac{P_{\infty} R^{2}}{D} \frac{A_{8}}{W_{0}}
$$

is a Damkohler number, defined as the ratio of the characteristic diffusion time $R^{2} / D$ to the characteristic time for chemical reaction, $\left(\rho_{\infty} A_{z} / W_{0}\right)^{-1}$.

As in the previous chapters, $\rho \mu=$ const., $\rho T=$ const. (equation of state), $\mathrm{Pr}_{\mathrm{r}}=$ const. and $\mathrm{Sc}=$ const. are assumed. The boundary conditions are essentially the same as in Chapter 3, with the additional conditions for zero oxidizer mass fraction 
at the droplet surface, i.e. $K_{0}(\eta=0)=0$, and zero fuel mass fraction in the free stream, i.e. $K_{f}(\eta \rightarrow \infty)=0$.

The momentum equation (4.8) and the energy equation (4.9) contain nonlinear terms that have to be quasi-linearized for the numerical solution of the set of ordinary differential equations in order to guarantee numerical stability. The linearization of the quadratic term in the momentum equation is given in eq. (2.31). Similarly, the exponential term in eq. (4.9) can be linearized as

$$
\frac{\exp (-E / \theta)}{\theta}=\left[\left[2-\frac{E}{\Theta}\right] \frac{\exp (-E / \theta)}{\theta}\right]^{*}-\left[\left[1-\frac{E}{\Theta}\right] \frac{\exp (-E / \theta)}{\Theta^{2}}\right]^{*} \theta+O\left[\left(\theta-\Theta^{*}\right)^{2}\right]
$$

where the superscript * indicates previous iteration values. Analogous to the infinite-rate solution, the set of one-dimensional ordinary differential equations (4.9) to (4.11) with the quasi-linearizations (2.31) and (4.15) is solved by central differencing at discrete angular positions along the droplet surface until boundary layer separation is reached.

\subsection{Results and Discussion}

Sample calculations have been carried out to obtain solutions using the finiterate formulation described in the previous section. Figure 4-1 shows calculated temperature, velocity, and species concentration profiles for an $n$-Heptane droplet of 50 $\mu \mathrm{m}$ radius at an initial liquid temperature of $325 \mathrm{~K}$, suddenly introduced into air at $P_{\infty}=1 \mathrm{~atm}, T_{-}=700 \mathrm{~K}$, at a relative Reynolds number of 200 . These values correspond to $\mathrm{M}_{\infty}=0.251$ and $\mathrm{Da}_{\infty}=42,341$. A reaction zone of finite thickness is 
predicted, with its center near $\eta=2.8$. It is also seen from Fig. $4-1$ that the maximum temperature lies slightly closer to the droplet surface than the approximate middle point of the flame zone. The results shown in this figure correspond to the angular position $\theta=60^{\circ}$, i.e. $60^{\circ}$ from the upstream stagnation point of the droplet, but similar profiles are obtained at other angular locations between $\theta=0^{\circ}$ and the separation point.

\subsubsection{Activation Energy}

A new parameter introduced in the finite-rate formulation of the governing equations is the activation energy $E_{2}$. For the calculation of Fig. 4-1 an activation energy of $E_{a}=5 \mathrm{~kJ} /$ mole is used, which can be considered relatively low. In fact, values given in the literature for the activation energy of hydrocarbon-oxygen reactions vary within a relatively broad range about the order of magnitude $E_{a}=O\left(10^{4} \mathrm{~J} /\right.$ mole $)$. The effect of the activation energy on the temperature profile is shown in Fig. 4-2. For these particular flow conditions, burning solutions are obtained for $E_{a} \leq 10 \mathrm{~kJ} / \mathrm{mole}$. On the other hand, there is practically no burning for $E_{\mathrm{a}} \geq 50 \mathrm{~kJ} / \mathrm{mole}$.

It is also interesting to see the effect of the activation energy on the mass fractions of fuel vapor and oxidizer, as well as on the thickness of the reaction zone. As seen in Fig. 4-3, the reaction zone becomes slightly broader as the activation energy increases, but is still low enough to allow burning. The mass fraction profiles for $\mathrm{E}_{\mathrm{a}}=50 \mathrm{~kJ} / \mathrm{mole}$ correspond to a near-frozen flow solution, except that the boundary condition for the oxidizer mass fraction, $k_{0}=0$ at droplet surface, is not correct for nonreacting flow. Strictly speaking, the conservation equations for all four species 
- fuel vapor, oxygen, nitrogen and combustion products - need to be solved with the appropiate boundary conditions to obtain the correct profiles for each of the latter three species as well as fuel vapor. An examination into the effect of this simplification will be presented in Section 4.5.

The fuel mass fraction at the surface decreases as the activation energy increases, or, as the reaction rate decreases. This result suggests that, as expected, the evaporation rate (or the mass blowing rate) at the surface will also decrease with reaction rate, which manifests itself in Fig. 4-4. It is also seen in Fig. 4-4 that higher reaction rates resuit in increased local shear stresses at the droplet surface. Here, two effects of the mass blowing rate on the velocity gradient at the surface seem to be competing. A higher blowing rate means, on the one hand, that more fuel vapor needs to be transported - hence larger velocity gradients along the droplet surface. On the other hand, the normal velocity component at the surface due to blowing tends to flatten the velocity gradient at the surface. The first effect seems to be stronger up to an angular position near $\theta=85^{\circ}$, the point where the three curves for the skin friction coefficient $c_{f}\left(R e_{\infty}\right)^{1 / 2}$ overlap. For $\theta>85^{\circ}$, then, apparently the second effect of higher blowing rates is stronger that the first effect. Consequently. the separation point moves closer to the droplet shoulder $\left(\theta=90^{\circ}\right)$ as the reaction rate increases.

\subsubsection{Fast versus Finite-Rate Chemistry}

In this section, a direct comparison is made between the tw'o models that treat chemistry differently. Fig. 4-5 shows the temperature and fuel mass fraction profiles obtained from the two models. "Model 1," represented by the dashed line, in the 
infinitely-fast chemistry model; and "Model 2," represented by the solid line, is the one-step, finite-rate chemistry model. To isolate the effect of the treatment of chemical kinetics, both models are run under the same conditions, i.e. $\mathrm{p}_{a}=1 \mathrm{~atm}$, $T_{\infty}=700 \mathrm{~K}, R e_{\infty}=100, R=50 \mu \mathrm{m}$ for steady-state evaporation and burning. Profiles shown in Fig. $4-5$ are taken at $\theta=60^{\circ}$. For both models, the average constant heat capacity is chosen to be $c_{p}=1.5 \mathrm{c}_{\mathrm{p}} \ldots$, and the heat release from the fiame $q=1 \times 10^{7}$ J/kg. In Model 2, where the activation energy $E_{a}$ needs to be specified, $E_{a}=5$ $\mathrm{kJ} / \mathrm{mole}$ is used.

Fig. 4-5 shows that, compared to the finite-rate representation, the fastchemistry approximation tends to predict a larger flame distance from the surface, and also a higher fuel vapor concentration at the surface. Model 2, i.e. finite-rate chemistry, on the other hand, gives a slightly higher flame temperature.

Fig. 4-6 shows the variation of burning rate and skin friction along the droplet surface. The prediction of Model 1 for burning rate is about about 10 percent higher than that of Model 2. In steady-state evaporation, where heat transfer into the liquid phase is zero, the local surface mass-blowing rate $\rho_{s} v_{s}$ is proportional to the temperature gradient at the surface. The detail inserted into Fig. 4-5, after close examination, shows that the temperature gradient at the surface calculated by Model 1 is indeed larger than that calculated by Model 2, by a factor of approximately 1.10. This is consistent with the higher blowing rate predicted by Model 2.

As for the skin friction coefficient $c_{f}$. Fig. 4-6 seems to suppont the argument that a higher blowing rate corresponds to a smaller velocity gradient, and, consequently, to a smaller shear stress at the surface. 


\subsection{A Criterion for Ignition}

The variation of the reaction rate with the angular position along the droplet surface can also be examined, based on the results of these calculations, and in conjunction with the ignition criterion developed by Kashiwagi and Summerfield (1972). Along the lines of the analysis of Kashiwagi and Summerfield, an integrated reaction rate,

$$
\int_{0}^{-}(R . R .) d y=\int_{0}^{\infty} \frac{p^{2} A_{1}}{W_{0}} K_{0} K_{f} \exp \left(-\frac{E}{\theta}\right) d y .
$$

is calculated along the droplet surface. The results for the nondimensional integrated

reaction rate, $\int_{0}^{\infty}(R . R) d y /.\left(p_{\infty} u_{\infty}\right)$, are shown in Fig. 4-7. Kashiwagi and Summerfield (1972), based on their experimental observations, postulate a critical value for ignition as

$$
\int_{0}^{\infty}(R . R .) d y=3.1 \times 10^{-2} \frac{\mathrm{kg}}{\mathrm{m}^{2} \mathrm{~s}}
$$

For our highest reaction rate considered, i.e. for $E_{a}=40 \mathrm{~kJ} / \mathrm{mole}$, the maximum dimensional value for the integrated reaction rate is equal to $2.48 \times 10^{-2} \mathrm{~kg} /\left(\mathrm{m}^{2} \mathrm{~s}\right)$ at the separation point. We note that for all three cases the maximum integrated reaction rate occurs near the separation point. According to the ignition criterion of Kashiwagi and Summerfield, this indicates that ignition is likely to start at a location near the separation point. This is consistent with the experimental observations of Teodorczyk and Wojcicki (1983). In these calculations, only cases of weak reaction are considered since ignition is not of interest any longer after vigorous buming has 
started.

Altough the present one-step, finite-rate chemical reaction model is more advanced than the fast-chemistry approximation, it is still a very crude representation of chemical reaction kinetics. In reality, typical combustion processes of practical interest such as the oxidation of hydrocarbons involve dozens of chemical reactions and species. Investigation of transient ignition phenomena within the framework of a time-dependent calculation would require a somewhat detailed representation of these chemical reaction mechanisms, which is outside the scope of the present work.

\subsection{An Improved Representation of Chemistry}

As already mentioned in Section 4.3.1, so far in this study the composition of the gas mixture in the boundary layer has been represented by three species - fuel vapor, oxidizer, and combustion products. Accordingly, conservation equations are solved for two species only, fuel and oxidizer (see eqs. (4.6), (4.7)), and the mass fraction of the third one follows from the condition that the sum of the mass fractions of all species must be equal to one. This treatment, obviously, introduces some error into the computation. The objective of the analysis presented in this section is to estimate this error.

The only change in the boundary layer equations is that we have to solve an additional species equation to solve, either for the products or for nitrogen. We choose nitrogen, the mass fraction of which is denoted by $K_{n}$, since it has a production rate of zero, and, therefore, easier to solve for. So we have in addition to the 
species equations (4.6) and (4.7),

$$
\begin{gathered}
\rho\left[u \frac{\partial K_{n}}{\partial x}+v \frac{\partial K_{n}}{\partial y}\right]=\frac{\partial}{\partial y}\left[\frac{\mu}{S c} \frac{\partial K_{n}}{\partial y}\right] . \\
K_{p}=1-\left(K_{f}+K_{0}+K_{n}\right),
\end{gathered}
$$

where the subscript $p$ refers to reaction products. We note that now the formulation of the boundary conditions for gas species is also different. In the presence of more than three species, one has to strictly formulate flux conditions, such that all species except fuel vapor must have zero flux at the gas-liquid interface.

A sample calculation is carried out to compare the results obtained from this improved model, which will be called Model 3, with those obtained from Model 2. These results are presented in Figs. 4-8 to 4-10.

Fig. 4-8 shows the temperature and mass fraction profiles obtained at $\theta=60^{\circ}$

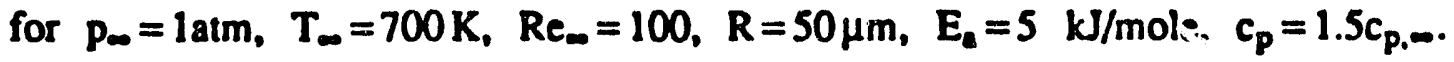
Model 3 predicts a higher flame temperature, a greater flame distance, and a higher mass fraction of fuel, than Model 2. These results correspond to slightly higher mass-blowing rates, as seen in Fig. 4-9.

The same figure shows that Model 3 predicts a significantly smaller skin friction coefficient than Model 2. In other words, solving three species conservation equations instead of two by accounting for $\mathrm{N}_{2}$ separately instead of lumping it with oxidizer and/or products as "one species" seems to make a significant difference in the predicted shear stress. The nondimensional skin friction coefficient, $c_{f}$, at a certain angular location, is given by 


$$
c_{f}=\text { (Const.) } \frac{d}{d \eta}\left[\frac{u}{u_{e}}\right]_{8} \text {. }
$$

which suggests an examination of the velocity profiles obtained from different models. Fig. 4-10 shows the velocity profiles across the boundary layer at $\theta=60^{\circ}$ - a location where the difference between the skin friction coefficients is quite pronounced, as seen in Fig. 4-8. Again, a close examination of the slopes of the two velocity curves near the surface is needed. The inserted detail in Fig. 4-10 shows that between the velocity gradients obtained from Models $\mathbf{3}$ and $\mathbf{2}$ there is indeed a difference of a factor of approximately 1.5, which explains the difference in the skin friction coefficients.

\subsection{Summary}

A one-step, finite-rate chemical reaction mechanism is integrated into the droplet model. Results obtained from finite-rate calculations are compared to fastchemistry results. In general, fast chemistry seems to give a larger flame distance from the surface, and a higher mass-blowing rate. As for surface friction, blowing reduces shear stress at the surface, and the drag on the droplet.

The ignition criterion of Kashiwagi and Summerfield (1972), based on the variation of the local integrated chemical reaction rate along the droplet surface, is applied to the present droplet model. Results indicate that ignition is likely to star at a location near the separation point, which is consistent with the experimental observations of Teodorczyk and Wojcicki (1983).

Finally, an improved representation of gas composition and chemistry is introduced to isolate the effect of lumping nitrogen with reaction products, i.e. assuming 
identical molecular weights for nitrogen and products. Results show that there is litthe change in the predicted mass-blowing rates. However, the difference in surface friction is significant. This comes from the difference between the surface velocity gradients predicted by the two different models - to which the corresponding shear stresses are proportional. As for flame characteristics, the improved chemistry seems to predict a higher fiame temperature and distance, as well as a thicker flame zone. 


\section{CHAPTER 5 \\ "EXACT" VARIATION OF GAS PROPERTIES IN THE BOUNDARY LAYER}

So far in the present work, variation of gas-mixture properties such as specific heat capacity $c_{p}$, viscosity $\mu$, thermal conductivity $\lambda$, and mass diffusivity $D$, within the boundary layer adjacent to the droplet surface was represented through the approximations

$$
\begin{aligned}
& c_{p}=\bar{c}_{p}=\text { const., } \\
& p \mu=p^{2} v=\text { const., } \\
& P r=\mu c_{p} / \lambda=\text { const. } \\
& S c=\mu /(p D)=\text { const. }
\end{aligned}
$$

These approximations are widely used in the combustion community, although their accuracy may be considered questionable in the presence of large temperature and density gradients in the boundary layer. Significant differences in the molecular weights of the species composing the gas mixture, combined with the existence of considerable concentration gradients near the surface and the reaction zone, also raise questions as to how appropriate the above approximations, i.e. eqs. (5.1) to (5.4), are for a given boundary layer problem. 
The present chapter of this work will be devoted to the examination of the accuracy of these commonly used approximations. Our aim is to arrive at conclusions with respect to burning rate, drag, and flame position. It is desirable that these conclusions are sufficiently broad to be useful for future research in the area of droplet combustion, and diffusion flames in general.

\subsection{Boundary Layer Equations}

As in Chapter 4, the chemical reaction kinetics will be represented by a onestep, finite-rate chemical reaction model, where the reaction rate $k$ is expressed through the Arrhenius law, given in eq. (4.2).

Since we wish to treat the thermodynamic properties of the gas mixture as variable quantities, it is useful to rewrite the boundary layer equations, eqs. (2.11), (2.12), (4.1), (4.6) and (4.7), in the following form:

$$
\begin{gathered}
\frac{\partial}{\partial x}\left(r_{s} \rho u\right)+\frac{\partial}{\partial y}\left(r_{s} \rho v\right)=0 \\
\rho\left[u \frac{\partial u}{\partial x}+v \frac{\partial u}{\partial y}\right]=\rho_{c} u_{c} \frac{d u_{c}}{d x}+\frac{\partial}{\partial y}\left[\mu \frac{\partial u}{\partial y}\right] \\
\rho c_{p}\left[u \frac{\partial T}{\partial x}+v \frac{\partial T}{\partial y}\right]=-\rho_{c} u_{e} \frac{d u_{c}}{d x} u+\frac{\partial}{\partial y}\left[\lambda \frac{\partial T}{\partial y}\right]+\mu\left[\frac{\partial u}{\partial y}\right]^{2}+\sum_{i=1}^{N}\left[\rho D_{i} \frac{\partial K_{i}}{\partial y} \frac{\partial h_{i}}{\partial y}-h_{i} \dot{w}_{i}\right] \\
\rho\left[u \frac{\partial K_{i}}{\partial x}+v \frac{\partial K_{i}}{\partial y}\right]=\frac{\partial}{\partial y}\left[\rho D_{i} \frac{\partial K_{i}}{\partial y}\right]+\dot{w}_{i v}, \quad i=f, 0, p, n .
\end{gathered}
$$

Equations (5.5) and (5.6) are the same as (2.11) and (2.12), respectively. The 
reaction under consideration in this chapter is the one-step oxidation of a hydrocarbon fuel in air, i.e.

$$
[\text { Fuel }]+\alpha\left[\mathrm{O}_{2}\right]+4 \alpha\left[\mathrm{N}_{2}\right] \rightarrow[\text { Products }]+4 \alpha\left[\mathrm{N}_{2}\right]
$$

or, more specifically, for an n-paraffin,

$$
\mathrm{C}_{\mathrm{m}} \mathrm{H}_{2 m+2}+\frac{3 m+1}{2} \mathrm{O}_{2}+2(3 m+1) \mathrm{N}_{2} \rightarrow m \mathrm{CO}_{2}+(m+1) \mathrm{H}_{2} \mathrm{O}+2(3 m+1) \mathrm{N}_{2}
$$

Thus, the subscript $i$ that refers to the $i-$ th species stands for $f$ (fuel), $o$ (oxygen), $n$ (nitrogen), or $\mathrm{p}$ (products), all in gaseous form. We note that the reaction products, $\mathrm{CO}_{2}$ and $\mathrm{H}_{2} \mathrm{O}$, will be treated together in the species equations. For the mass fraction in particular, this means

$$
K_{\mathrm{p}}=\mathrm{K}_{\mathrm{CO}_{2}}+\mathrm{K}_{\mathrm{H}_{2} \mathrm{O}}
$$

This can be done since the reaction is assumed to be in equilibrium, i.e. the stoichiometric coefficients, given in eq. (5.9), are assumed to hold everywhere in the flowfield.

In eq. (5.7), $h_{i}$ denotes the specific enthalpy of the $i-$ th species, given by

$$
h_{j}=\int_{T}^{T} c_{p, i} d T+\Delta h l_{, i} .
$$

where the superscript o denotes the standard reference state, and $\Delta h_{\mathcal{L}, i}$ is the enthalpy of formation for the $i$-th species. The specific heat capacity of the mixture, $c_{p}$, is given by

$$
c_{p}=\sum_{i=1}^{N} c_{p i} K_{i}, \quad i=f, 0, p, n .
$$

where $c_{p, i}$ is the specific heat capacity of the $i-$ th species. 
The mass production rates for the four species are given as:

$$
\begin{gathered}
\dot{w}_{f}=-\frac{p^{2} A_{l}}{w_{0}} K_{0} K_{f} \exp \left[-\frac{E_{a}}{R_{u} T}\right], \\
\dot{w}_{0}=\frac{1}{\dot{w}_{f}}, \\
\dot{w}_{p}=-\left(\dot{w}_{f}+\dot{w}_{0}\right)=-\left[1+\frac{1}{f^{*}}\right] \dot{w}_{f} .
\end{gathered}
$$

where ${ }^{*}=W_{\mathrm{g}} /\left(\alpha W_{0}\right)$, is the stoichiometric fuel-oxidizer consumption ratio, and, since $\mathrm{N}_{2}$ does not enter the chemical reaction,

$$
\dot{w}_{\mathbf{n}}=0 \text {. }
$$

As in Chapters 2 and 4, these equations are transformed into a set of ordinary differential equations using the Lees transformation:

$$
\begin{aligned}
& {\left[C(\eta) F^{\prime}(\eta)\right]^{\prime}+f(\eta) F^{\prime}(\eta)+\beta(\xi)\left[\frac{W_{e}}{W} \Theta(\eta)-F^{2}(\eta)\right]=0,} \\
& {\left[\frac{C(\eta)}{P_{r}} \Theta^{\prime}(\eta)\right]^{\prime}+\left[f(\eta) \frac{c_{p}{ }^{\prime}}{c_{p}} \frac{C(\eta)}{P_{r}}+\sum_{i=1}^{N} \frac{C(\eta)}{S c_{i}} \frac{c_{p, i}}{c_{p}} K_{i}{ }^{\prime}(\eta)\right] \Theta^{\prime}(\eta)} \\
& +\left[\left[1+\frac{T_{c}}{c_{p, e}} \frac{d c_{p, e}}{d T_{e}}\right]^{-1}-\frac{c_{p, e} W_{e}}{c_{p} W}\right]\left(\gamma_{e}-1\right) M_{c}^{2} \beta(\xi) F(\eta) \Theta(\eta) \\
& =-C(\eta) \frac{c_{p, e}}{c_{p}}\left(\gamma_{c}-1\right) M_{c}^{2}\left[F^{\prime}(\eta)\right]^{2}-\frac{W}{W_{c}} \frac{\Delta_{c} Q_{c}}{T^{*}} K_{f}(\eta) K_{0}(\eta) \frac{\exp [-E / \Theta(\eta)]}{\Theta(\eta)} \text {. } \\
& {\left[\frac{C(\eta)}{S c_{f}} K_{f}^{\prime}(\eta)\right]^{\prime}+f(\eta) K_{f}^{\prime}(\eta)-\frac{W}{W_{e}} \Delta_{c} K_{0}(\eta) \frac{\exp [-E / \Theta(\eta)]}{\Theta(\eta)} K_{f}(\eta)=0 .}
\end{aligned}
$$




$$
\left[\frac{C(\eta)}{S c_{0}} K_{0}^{\prime}(\eta)\right]^{\prime}+f(\eta) K_{0}^{\prime}(\eta)-\frac{W}{W_{e}} \frac{\Delta_{e}}{f^{*}} K_{f}(\eta) \frac{\exp [-E / \Theta(\eta)]}{\Theta(\eta)} K_{0}(\eta)=0
$$

$$
\begin{gathered}
{\left[\frac{C(\eta)}{S c_{n}} K_{n}(\eta)\right]^{\rho}+f(\eta) K_{n}^{\prime}(\eta)=0} \\
K_{p}(\eta)=1-\left[K_{f}(\eta)+K_{0}(\eta)+K_{n}(\eta)\right]
\end{gathered}
$$

In the above equations, the newly introduced parameters are defined as

$$
\begin{gathered}
C(\eta)=\rho \mu /\left(p_{e} \mu_{e}\right), \\
S c_{i}(\eta)=\mu /\left(\rho D_{i}\right), \quad i=f, 0, n, p, \\
\Delta_{e} \equiv \frac{2 \xi A_{\tau}}{\mu_{e} u_{e}^{2} r_{s}^{2} W_{0}}, \\
Q_{e}=\frac{f^{*} q}{c_{p} T_{e}}=\frac{T^{*}\left(h_{f}+h_{o} / f^{*}+(1+f) h_{p} / f^{*}\right)}{c_{p} T_{e}},
\end{gathered}
$$

where, for the sake of consistency, the nondimensional parameters $\Delta_{c}$ and $Q_{c}$ are defined the same way as in Chapters 4 and 2, respectively.

Finally, as in previous chapters, the nonlinear terms in the momentum equation (5.13), and the energy equation (5.14), are quasi-linearized. The linearized form of these nonlinear terms are given in eqs. (2.31) and (4.17), and shall not be repeated here.

\subsection{Thermophysical Properties}

The variation of the transport properties, i.e. viscosity, thermal conductivity and mass diffusivity, with temperature and composition of the gas mixture is evaluated 
according to the Chapman-Enskog kinetic theory of gases, as outlined in Edwards et al. (1979). The Chapman-Enskog Theory, together with the Lennard-Jones 6-12 Potential Model for the potential energy of intermolecular collisions, yields the following formula for the viscosity of the i-th species:

$$
\mu_{i}=2.67 \times 10^{-6} \frac{\left(W_{i} T\right)^{1 / 2}}{\sigma_{i}^{2} \Omega_{\mu}} .
$$

where $T$ in Kelvins gives $\mu_{i}$ in Ns/m $\mathrm{m}^{2} . \sigma_{i}$, given in Angstroms $\left(1 \mathrm{~A}=10^{-10} \mathrm{~m}\right)$, denotes the so-called "force constant," and $\Omega_{\mu}$ denotes the "collision integral" for the Lennard-Jone: potential. $\sigma$ for various gases, and $\Omega_{\mu}$ as a function of temperature, can be found in the literature. Values used in the present study are given in Tables 5-1 and 5-2 (for n-Heptane, $\varepsilon / k=282.0 \mathrm{~K}, \sigma=8.88 \mathrm{~A}$ are used, after Hirschfelder et al. (1954)).

Thermal conductivity for a monatomic gas is related to viscosity,

$$
\lambda_{i}^{\text {mon }}=\frac{15}{4} \frac{R_{u}}{W_{i}} \mu_{i}
$$

And for polyatomic gases,

$$
\lambda_{i}=\lambda_{i}^{\text {mon. }}+1.32\left[c_{p, i}-\frac{5}{2} \frac{R_{u}}{W_{i}}\right] \mu_{i} .
$$

The viscosity and the thermal conductivity of a gas mixture can be estimated from the values for the pure species that are calculated from egs. (5.24) and (5.25). A simplified, but sufficiently accurate mixture formula based on the kinetic theory of gases was developed by Wilke (1950): 


$$
\begin{aligned}
& \mu=\left[\sum_{i=1}^{N} \mathbf{X}_{i} \mu_{i}\right] /\left[\sum_{j=1}^{N} X_{j} \Phi_{i j}\right], \\
& \lambda=\left[\sum_{i=1}^{N} X_{i} \lambda_{i}\right] /\left[\sum_{j=1}^{N} X_{j} \Phi_{i j}\right] .
\end{aligned}
$$

where $X_{i}$ is the molar fraction of the $i-t h$ species, and

$$
\Phi_{i j}=\frac{\left[1+\left(\mu_{i} / \mu_{j}\right)^{1 / 2}\left(W_{j} / W_{i}\right)^{1 / 4}\right]^{2}}{\left[8\left(1+W_{i} / W_{j}\right)\right]^{1 / 2}}
$$

The procedure for obtaining the mass diffusivity $D_{i}$ of the $i-t h$ species is different, since it involves the binary diffusion coefficients $D_{i j}$, describing the diffusivity of species $i$ in a binary mixture of species $i$ and $j$. Again, from the Chapman-Enskog theory, $D_{i j}$ is given by

$$
D_{i j}=1.86 \times 10^{-7} \frac{\left[T^{3}\left(W_{i}+W_{j}\right) /\left(W_{i} W_{j}\right)\right]^{1 / 2}}{\sigma_{i j}^{2} \Omega_{D} P}
$$

Here, $T$ in Kelvins, $p$ in atm., and $\sigma$ in Angstroms yield $D_{i j}$ in $\mathrm{m}^{2} / \mathrm{s}$. The collision integral $\Omega_{D}$, as $\Omega_{\mu}$, can be found in the literature (see Table 5-2), and the average force factor $\sigma_{i j}$ is obtained from the empirical relation (see Edwards et al. (1979))

$$
\sigma_{i j}=\frac{\sigma_{i}+\sigma_{j}}{2}
$$

Finally, the mass diffusivity of the $\mathrm{i}-\mathrm{th}$ species in a mixture of $\mathrm{N}$ species can be approximated as

$$
D_{i}=\frac{1-X_{i}}{\sum_{j=i}\left(X_{j} / D_{i j}\right)}
$$


under the assumption that the $i$-th species is dilute in the mixture, i.e. $X_{i}$ is sufficiently smaller than one (Edwards et al. (1979)). Even though this condition cannot be satisfied for all species in our case, eq. (5.29) is used to estimate $D_{i}$, to avoid extensive and involved calculations just to obtain $D_{i}$.

\subsection{Results and Discussion}

Sample calculations are carried out for the steady-state buming of n-Heptane droplets at two different free-stream temperatures, and the results are compared with results obtained from various experimental studies, as well as with results obtained using simplified, "model" property variation, as given by eqs. (5.1) to (5.4), for both infinitely fast chemistry and one-step, finite-rate chemistry.

\subsection{1. "Exact" versus "Model" Properties}

Fig. 5-1 shows the typical variation of some gas mixture properties across the boundary layer. Specific heat capacity $c_{p}$. Prandtl number $\mathrm{Pr}$, and the product of density and viscosity $\rho \mu$, all nomalized with their respective values at the boundary layer edge, are plotted against the nondimensional normal distance from drop surface. This particular graph corresponds to $\theta=60^{\circ}$ in terms of the angular distance from the forward stagnation point, but profiles at other angular locations between the forward stagnation point and the separation point are found to be similar. In the simplified representation for gas properties, $c_{p}$. Pr and $\rho \mu$ are all assumed to be constant. It seems that the assumption $p \mu=p_{c} \mu_{c}=$ const. gives an error of about 30-35 percent near droplet surface at the given free stream conditions $\left(p_{\infty}=1 \mathrm{~atm}, T_{-}=300 \mathrm{~K}, R e_{\infty}=100\right)$. At this point, it is interesting to compare this 
vith results obtained at a higher temperature $\left(\mathrm{T}_{\infty}=700 \mathrm{~K}\right)$.

Fig. 5-2 shows that, for this case, the error hardly exceeds 15 percent. Based on these observations alone, it appears that, at least at higher free-stream temperatures, the assumption $p \mu=p_{e} \mu_{e}=$ const. is not very inaccurate despite the existence of the temperature and concentration gradients in the flowfield. The assumptions $\mathrm{C}_{\mathbf{p}}=$ const. $\mathrm{Pr}=$ const., however, may potentially introduce a significant error in the determination of flame characteristics and surface transport rates, which will be investigated in the following.

First, we wish to examine the effect of the choice of the average specific heat, $\bar{c}_{p}$, on fiame and transport characteristics. Figs. 5-3 to 5-5 show the effect of $\bar{c}_{p}$ in the simplified-property model. The two values chosen for $\overline{\mathbf{C}}_{\mathrm{p}}, 1363$ and $1666 \mathrm{~J} / \mathrm{kg}-\mathrm{K}$, are the values for $c_{p}(\eta)$ at the flame (i.e. where the gas temperature is maximum) and at the surface of the droplet, respectively, obtained from the "exact" calculation. Furthermore, a constant heat release $q=6.43 \times 10^{6} \mathrm{~J} / \mathrm{kg}$, and a constant activation energy $E_{a}=5 \mathrm{~kJ} / \mathrm{mole}$ are used for both. The former is the calculated heat release at the flame, i.e. where the temperature is a maximum, obtained from the "exact" calculation. As for $\mathrm{E}_{\mathrm{a}}$, it needs to be specified in both models, and the above mentioned value is chosen for both models.

Both simplified models seem to predict a higher flame temperature and a higher flame distance. At least the first value for $\bar{c}_{\mathrm{p}}, 1363 \mathrm{~J} / \mathrm{kg}-\mathrm{K}$, is definitely lower than the effective average $c_{p}$ in the region between the flame and the droplet surface. A lower $\overline{\mathbf{c}}_{\mathrm{p}}$ leads to a higher flame temperature, which explains the trend in peak temperatures. 
As for the flame distance from the surface, a higher flame temperature, i.e. a higher heat release from the flame, would lead to increased blowing, which, in its turn, would push the fiame furher away from the surface. This is seen in Fig. 5-4, 100, where the mass-blowing rates from the two simplified models (larger flame distance) are clearly higher than the mass-blowing rates obtained from the "exact" calculation. The question, however, why a higher $\bar{c}_{p}$, i.e. a lower flame temperature, corresponds to a higher blowing rate, still needs to be answered.

The present computations were performed for steady-state evaporation, i.e. under the condition of zero heat transfer into the liquid phase. This means that the surface mass-blowing rate at a certain angular location is directly related to the gasphase heat transier at the surface through the equation

$$
P_{s} v_{s}=\frac{\lambda_{s}}{h_{v}\left(T_{s}\right)}\left[\frac{\partial T}{\partial y}\right]_{s}
$$

or, in non-dimensional form,

$$
\frac{\rho_{s} v_{s}}{\rho_{\infty} u_{\infty}}=\text { (Const.) } \frac{\rho_{s} \lambda_{s}}{h_{v}\left(T_{s}\right)} \frac{d}{d \eta}\left[\frac{T}{T_{e}}\right]_{8} \text {. }
$$

As seen in Fig. 5-3, the surface temperature, and hence the latent heat of vaporization $h_{v}\left(T_{s}\right)$, are almost equal for all three cases. From this graph it is difficult to distinguish the gradients of $T / T_{e}$ for the three cases from each other. The calculated values seem to be very close to each other. A closer look at the remperature gradients near the droplet surface, as seen in the detail inserted in the lower right-hand corner of Fig. 5-3, however, shows that the values for $d\left(T / T_{e}\right) / d \eta$ at the surface are almost identical for the "exact" calculation and for the model-propenty calculation 
with $\overline{\mathrm{C}}_{\mathrm{p}}=1666 \mathrm{~J} / \mathrm{kgK}$, while the value for the same gradient, calculated with model properties at $\bar{c}_{\mathrm{p}}=1363 \mathrm{~J} / \mathrm{kg}-\mathrm{K}$ is about 15 percent larger than the other two.

As for $p_{3} \lambda_{3}$, in the simplified treatment of gas properties we have

$$
p_{3} \lambda_{3}=\frac{\rho \mu}{\operatorname{Pr}} c_{P}=\text { const. }
$$

which follows directly from the assumptions given in egs. (5.1) to (5.3).

The variaton across the boundary layer of properties other than $c_{p}$ being the same between the two simplified models, it is obvious that a higher $\bar{c}_{\mathrm{p}}$ will result in a larger value of $p_{s} \lambda_{3}$. Then, it follows from eq. (5.31) that the mass-blowing rate will be proportional to the product of $p_{3} \lambda_{3}$ and the temperature gradient at the surface. Thus, in the simplified models, the change in blowing rate will result from two competing effects - one of them, the product $p_{s} \lambda_{s}$, increasing with $\bar{c}_{p}$, and the other, the temperature gradient at the surface, decreasing with $\overline{\mathrm{p}}_{\mathrm{p}}$.

As for the "exact" blowing rate result, if one forms the ratio of blowing rates between the "exact" calculation and that with $\bar{c}_{p}=\left(c_{p, s}\right)_{\text {exact }}=1666 \mathrm{~J} / \mathrm{kg} \cdot \mathrm{K}$, one obtains, using eq. (5.32),

$$
\frac{\left(p_{s} v_{s}\right)_{\text {exact }}}{\left(p_{s} v_{s}\right)_{\text {simp. }}}=\frac{\left(P_{s} \mu_{s}\right)_{\text {exact }}}{P_{\infty} \mu_{\infty}} \frac{P_{-\infty}}{\left(P_{s}\right)_{\text {exect }}}=0.81
$$

which explains the discrepancy between the blowing rate results obtained from the "exact" calculation and the "model" calculation. This is confirmed by Fig. 5-2, 100.

These findings suggest that, using surface values for gas properties in the simplified-property calculation would, in general, give more reliable results in terr.s of mass blowing, or evaporation, rates. The same, however, cannot be said for flame 
temperature and flame distance predictions, or skin friction predictions (see Fig. 55). Herein lies a potential shortcoming of the approximate treatment of gas properties in the boundary layer calculation. That is, a simultaneous prediction of physical processes at the surface and in the reaction zone are, in general, not possible to the same degree of accuracy with such simplified property-variation models.

Law and Williams (1972) also argue that the significant gas properties affecting evaporation rate are specific heat capacity $c_{p}$ and thermal conductivity $\lambda_{\text {, both }}$ evaluated near droplet surface. They report that their empirical correlation, based on average values of $c_{p}$ and $\lambda$ in the region between the flame and the droplet surface. yields good agreement with experimental observations.

There is one important gas property, however, which, so far, has not been subjected to a close examination - mass diffusivity $D$. One difficulty of analyzing the effect of diffusivity is that there are several diffusivities, one for each species, involved in the problem - which has been taken into account in the "exact" calculation. In the simplified model, however, the Schmidt number Sc, given by eq. (5.4), is constant, and, consequently, from eq. (5.2), the quantity $P^{2} D$ is a constant. The variation of the diffusivities of fuel vapor and oxygen across the boundary layer is shown in Fig. 5-6. Here, the diffusivities are expressed in terms of Lewis numbers, defined as

$$
L c_{i}=\frac{S c_{i}}{P r}=\frac{\lambda}{\rho c_{p} D_{i}}, \quad i=f, O, C O_{2}, H_{2} O, N_{2} .
$$

For evaporation (or burning) rates, it is plausible to think that the significant diffusivities will be the diffusivity of fuel vapor in the region between the surface and the flame, or, that of oxygen far from the droplet, i.e. in the region between the 
fiame and the boundary layer edge. Fig. 5-6 shows that the latter corresponds to a Lewis number very close to unity. As for the fuel vapor Lewis number, it experiences a sharp change over a factor of five between surface and flame zone. Thus the effective average Lewis number for fuel vapor in that region will be somewhere around the value 2 .

The effect of the choice of Lewis number in simplified-property calculations is shown in Figs. 5-7 to 5-9. In these calculations, surface values from the "exact" model are used for the constants $\overline{\boldsymbol{C}}_{\mathrm{p}}$. Pr, and $p \mu$, which means that $p \lambda$ is also evaluated at droplet surface. In all three figures, the results are also compared with the "exact" results.

As seen in Fig. 5-7, a higher Lewis number, or, a lower mass diffusivity, results in a lower fiame temperature, and a smaller flame distance from the surface. It is significant that a Lewis number of unity predicts almost the same surface fuel concentration as the "exact" model, whereas a higher Lewis number overpredicts the surface fuel concentration, but comes closer to the "exact" calculation as far as the shape, or the gradient, of the fuel concentration profile is concemed. A higher Lewis number gives a better prediction for the local mass blowing rates along droplet surface, 100 (see Fig. 5-8). In terms of skin friction, however, the unity Lewis number, which gives an almost identical result as the "exact" calculation, seems to be the better choice, although, obviously, the difference in Lewis number does not result in a significant change in predicted surface friction (see Fig. 5-9).

Finally, we wish to compare the results from all four different models that have been developed in the course of the present study: the model with three species conservation equations and "exact" property variation (Model 4); that with three species 
conservation equations and "model" property variation (Model 3); that with two species conservation equations and model property variation (Model 2); and finally, the one with infinitely fast chemical kinetics, and, of course, with model property variation (Model 1). Figs. 5-10 to 5-12 show the comparison in terms of temperature and mass fraction profiles, mass blowing rate and skin friction. For Models 1 to 3 , the following constants are used: $\bar{C}_{\mathrm{p}}=1363 \mathrm{~J} / \mathrm{kg} \cdot \mathrm{K}, \rho \mu=P_{c} \mu_{c}, P_{T}=P_{T_{e}}, L e=1$. Furthermore, the same constant heat release $q=6.43 \times 10^{6} \mathrm{~J} / \mathrm{kg}$ is used for all three models, and the same constant activation energy $E_{a}=5 \mathrm{~kJ} / \mathrm{mole}$ is used in the models 2 through 4 , where the latter needs to be specified.

Since comparisons between the Models 1 and 2, Models 2 and 3, and Models 3 and 4 are already made previously in this study, we shall not go into a detailed discussion at this point. However, it is interesting that, as seen in Figs. 5-10 and 5-11, the best agreement to Model 4 in terms of flame temperature, flame distance, and mass-blowing rate is reached by Model 2, and not by Model 3, which is closest to Model 4 in the degree of complexity. This means that, the two differences between Models 2 and 4, i.e. solving one additional species equation and accounting for the "exact" variation of gas properties, exert opposite effects on temperature profiles and evaporation rates.

As for surface friction, solving three species equations instead of two by accounting for $\mathrm{N}_{2}$ separately instead of lumping it with oxidizer and/or products as one species, seems to make a significant difference in the predicted skin friction (see Fig. 5-12). A discussion of this phenomenon was given in Section 4.5, when Models 2 and 3 were compared.

\subsubsection{Comparison with Experiments}


So far, comparison has been made between computational models that involve gas-property and reaction-chemistry models of varying degrees of complexity. Nothing has been said, however, concerning the agreement of these models, or of the most complex one, with experimental results. As mentioned in previous chapters, experimental results exist for overall steady-state evaporation rates from drops, and for the drag on evaporating drops. Fig. 5-13 shows the results for the steady-state evaporation rate from the droplet varying with the free-stream Reynolds number, as predictel by the "exact" model. Comparison is made with the experimental results of Law and Williams (1972), and with a Frossling/Ranz-Marshall type of correlation (see eq. 1.4). In the latter, surface values obtained at the forward stagnation point from the present "exact" calculation are used. In other words, the comparison with the Ranz-Marshall correlation is to be understood as an examination as to how accurate it would be as a short-hand representation of the present boundary-layer calculation. The Ranz-Marshall correlation underpredicts the results of the boundary-layer model, but considering that it was developed from observations on droplets in the absence of buming, this finding is not surprising. The experiment of Law and Williams (1972), is for burning dropiets. Evaporation rates predicted by our model seem to be about twice as large as those of the latter.

Since Law and Williams (1972) conducted their experiments at room temperature, much lower than $T_{\infty}=700 \mathrm{~K}_{0}$ which we used for these calculations, an additional calculation was performed at $T_{\infty}=300 \mathrm{~K}$. This time, the agreement with the results of Law and Williams is much better, as seen in Fig. 5-14, which is very encouraging. At this free-stream temperature, a Ranz-Marshall type of correlation that involves surface values calculated at the stagnation point also seems to provide a good representation for our computation. 
Finally, the results of these steady-state, quasi-time-dependent computations in terms of droplet radius and droplet Reynolds number histories at $\mathrm{T}_{\infty}=300 \mathrm{~K}$ are shown in Fig. 5-15. The initial droplet radius and Reynolds number were chosen to be $50 \mu \mathrm{m}$ and 150, respectively. These results, obtained from the "exact" model, are compared to results obtained from a time-history calculation based on experimental results. The drag correlation of Yuen and Chen (1976) is used to update relative droplet velocity at each time step. To update droplet radius, the correlation of Law and Williams (1972) is used. Our "exact-property" model slightly underpredicts droplet size and somewhat overpredicts droplet Reynolds number. In particular, the difference between the two Reynolds number predictions can probably be attributed to the fact that the Yuen-Chen correlation is based on data from evaporating, not burning. droplets. But, overall, the agreement is quite reasonable and satisfactory.

\subsection{Summary}

In this chapter, an examination of the accuracy of commonly used simplifications for the variation of gas properties is carried out. As opposed to a fully numerical analysis, the present model based on boundary layer approximation enables, within reasonable computational times, consideration of the variation of gas properties in the flowfield.

Results show that gas properties that are often assumed to be constant in combustion calculations, such as specific heat $c_{p}$. Prandil number $P_{r}$, and effective Schmidt number Sc, may vary by a factor of up to 3 or 4 within the region between the flame and the droplet surface. It is, therefore, important what value is chosen in these simplified calculations for $c_{p}, P r$ and Sc. In general, surface values, or aver- 
ages for the region between the surface and the flame zone, are recommended for a better estimation of surface rates such as mass-blowing rate and surface friction. For a better prediction of flame characteristics, however, average values for gas properties should be evaluated near the flame zone. This, of course, makes it difficult to achieve simultaneous agreement between simplified and exact property calculations in terms of evaporation rates and flame characteristics.

The results obtained from the present droplet model with exact property variation are also compared with experimental results. In terms of evaporation rates, our predictions are in good agreement with the correlation of Law and Williams (1972), given that the latter is evaluated at the conditions under which these experiments were conducted. This correlation, combined with the correlation suggested by Yuen and Chen (1976) for droplet drag, can be used to calculate droplet histories for size and Reynolds number. Again, under the appropriate free-stream conditions, there is good agreement between our predictions for droplet histories, and those based on the above-mentioned correlations. 


\section{CHAPTER 6 CONCLUSIONS}

\subsection{Boundary Layer versus Fully Numerical Modeling}

Since the late seventies, there has been extensive modeling work on convective single droplet evaporation and burning. Ressearchers have gone two separate ways in approaching this complex fluid mechanics problem that involves multiple phases and multiple transport processes - numerical solution of the full Navier-Stokes equations, or, an analytical/numerical approach along the lines of the boundary-layer approximation. Even with the computing capacity that is available to the engineering and science community today, a complete numerical solution of Navier-Stokes equations accounting for all aspects and details of the problem is still not possible. The reader is also referred to the discussion of this subject by Dwyer (1989). In other words, what is referred to here as "fully numerical" approach has - so far at least - also involved simplifying assumptions and approximations, in general with regard to interface transport phenomena, property variation in the gas and liquid phases, chemical kinetics in the case of burning, etc. To say this, of course, is not to underestimate the extensive and very useful work of such researchers as Dwyer and Sanders, and Renksizbulut and co-workers over the past decade. 
The basic shortcomings of the boundary-layer approach, on the other hand, are quite obvious. By its nature, the boundary layer approximation restricts the range of Reynolds numbers for which the analysis is valid, for it a priori assumes high Reynolds numbers. The Reynolds numbers associated with the convective single droplet evaporation and burning problem are observed to fall into the "intermediate" or "moderate" range, that is, $O(100)$ typically, rendering questiorable any useful implementation of the boundary-layer approximation for this problem. A strong case for a boundary-layer analysis, however, was made by the work of Prakash and Sirignano (1980). Their results, essentially, confirmed the suggestion that reasonable agreement with experimental observations was possible for $\mathrm{Re}=\mathrm{O}(100)$, the Reynolds number being based on droplet diameter.

A further shortcoming of the boundary-layer approach is its incapability of providing results for the wake region downstream of the droplet. The error introduced by the failure of solving for the wake region is relatively small in the case of the overall evaporation rate from the droplet, since it is observed (and shown computationally) that evaporation beyond the separation point can be assumed to be negligible compared to that in the region from the forward stagnation point and the separation point (see, e.g., Clift et al. (1978)). In the case of the droplet drag, however, the same cannot be said. Droplet drag consists of two components, drag due to pressure, and drag due to surface friction. Again, surface friction in the wake region can be shown to be much smaller than, say, around the forward stagnation point of the droplet. But since the contribution of the pressure drag to the total drag is of the same order as - usually even greater than - the contribution of the friction drag, drag calculation by the boundary-layer approach is, generally speaking, much more unreliable than that by a full solution of the Navier-Stokes equations. 
In the present work, the boundary-layer approach was chosen as the method of studying droplet evaporation, and, especially, burning. Table 6-1 summarizes the extent of some of the most significant and innovative research in this area. As seen in this table, the case involving chemical reaction has been studied by only two of

the six former researchers. And of those two, both have left out some other aspect of the problem, such as transient heating of the droplet interior and/or a consideration of gas-property variation in the flowfield surrounding the droplet. To our knowledge, the present work has been the only one so far employing the boundarylayer approach to combine the burning of the droplet with the transient heating of the droplet. The interesting result of the transient calculation, concerming the use of an appropriate effective transfer number, will be discussed in Section 6.3.

And as for taking into account the exact variation of gas properies in the flowfield, again, to our knowledge the present work offers so far the only analysis of that kind involving more than two gas species, and chemical reaction. That, also, will be discussed more in detail, in Section 6.5 .

\subsection{Steady-State Burning: Flame Position and Droplet Histories}

In general, the steady-state calculation outlined in Chapter 2 serves as a verification of the suitability of the present boundary-layer model for the prediction of the physical processes associated with single droplet evaporation and buming in a convective environment. In particular, evaporation rates from evaporating and burning droplets predicted by the present model are found to be in reasonable agreement with experimental observations, given in the form of empirical correlations (e.g. Froessling/Ranz-Marshall type of comelations). Furthernore, predicted life hisiories 
of evaporating/buming droplets appear to be within the same degree of accuracy as fully numerical solutions of any degree of complexity thus far have been able to obtain.

And finally, reasonable predictions of flame positions up to the point of boundary layer separation is possible with this model. The apparent relative thickness of the viscous layer - where also the chemical reaction is assumed to take place does call the boundary layer approximation in question, especially for Re $\leq 100$. Yet this does not seem to keep the results from being sufficiently reasonable. This, of course, was expected in the light of the important work by Prakash and Sirignano (1980).

\subsection{An Errective Transfer Number for the Entire Droplet Lifetime}

The most significant result of the transient calculation, outlined in Chapter 3 , has been the concept of an effective transfer number B that can be used to satisfactorily describe the evaporation behavior of a buming droplet both in the early phase of transient liquid-phase heating, and the phase of quasi-steady evaporation.

Empirical correlations for steady-state evaporation rates from droplets in a convective flowfield, such as Ranz-Marshall (1952) and Renksizbulut-Yuen (1983a), involve a nondimensional transfer number $B$. In the literature, B can be found defined as a mass transfer number.

$$
B_{m}=\frac{K_{f, s}-K_{f_{1, \infty}}}{1-K_{f_{0} s}}
$$

or a heat transfer number, 


$$
B_{\mathrm{uh}}=\frac{C_{p}\left(T_{-}-T_{s}\right)}{h_{v}}
$$

Eq. (6.2) is valid for steady-state evaporation only, i.e. when there is no heat transfer from the gas phase into the liquid phase, and the entire heat transfer is used for the phase change. For the case of nonzero heat transfer into the liquid phase, a modified, or effective, latent heat of vaporization can be defined as

$$
h_{v_{,} \text {eff }}=\dot{\mathrm{Q}}_{8} / \dot{\mathrm{m}}=h_{\mathrm{v}}+\dot{\mathrm{Q}}_{\mathrm{g}} / \dot{\mathrm{m}} \text {. }
$$

Used in the transfer number, $h_{v, \text { eff }}$ would give an effective transfer number

$$
B_{\text {th, eff }}=\frac{c_{p}\left(T_{-}-T_{3}\right)}{h_{v, \text { eff }}}
$$

which, for the special case of unity Lewis number, is equal to $B_{m}$ given by eq. (6.1).

This effective transfer number was used in Ranz-Marshall type of correlations that were compared to our computation results, both in reacting and non-reacting cases. Both Froessling/Ranz-Marshall and Renksizbulut-Yuen correlations turned out to be good predictors for droplet evaporation behavior for the entire droplet lifetime, and for both burning and evaporation only. This is remarkable, because these empirical correlations were based on observations made with droplets evaporating in steady-state, in the absence of chemical reaction. The significance of this finding lies in its potential usefulness for spray calculations, which is, of course, the rationale behind single droplet modeling.

\subsection{Finite-Rate versus Infinite-Rate Chemistry}


One advantage of the boundary-layer model here, as opposed to that of, e.g., Prakash and Sirignano (1980), is that it can accomodate chemical reaction. In the present work, calculations are performed with both an infinitely-fast and a one-step, finite-rate representation of chemical kinetics. This enables a comparison of the two kinetic models in terms of surface mechanisms such as mass-blowing rates and friction, as well as flame characteristics. These results, furthermore, can be extended to more general diffusion fiame and reacting boundary layer problems.

The results show that, in general, infinite-rate chemical kinetics give a larger flame distance from the surface, and a higher mass-blowing rate. It can be argued that higher blowing rates would push the flame further away from the surface, but, at the same time, this could result in a smaller temperature gradient at the surface, in its tum reducing the blowing rate. Thus the rate of blowing and the flame distance and temperature are determined by the balance of competing effects, and it is not possible to arrive at a generalized conclusion valid for all diffusion flame problems. As for surface friction, it is verified that blowing in general reduces surface friction even though, under some circumstances, the fact that increased blowing means more mass to be transported, may provide a competing effect.

The diffusion-fiame approximation with infinitely fast chemistry, where the flame zone is reduced to a flame sheet of zero thickness, allows for three gas species only: fuel vapor, oxidizer, and reaction products. In order to isolate the effect of finite-rate chemistry versus fast chemistry, the existence of these three species only was presumed for the former, 100. For most cases of practical interest, however, non-reacting species are also present in the flowfield, such as nitrogen, when combustion is taking place in air. To examine the effect of lumping nitrogen with 
reaction products, another, slightly more complex one-step, finite-rate computation was carried out by solving an additional species conservation equation for nitrogen. The results show that there is little change in the predicted mass-blowing rates. As for surface friction, however, the difference is significant, amounting to a factor of approximately 1.5. This comes directly from the difference between the surface velocity gradients predicted by the two models. Since the friction itself brings usually less than half of the contribution to the total drag, however, the effect on total droplet drag will be somewhat reduced. In terms of the prediction of flame characteristics, the more complex chemistry model seems to give slightly larger values for flame temperature, flame distance from the surface, and the thickness of the flame zone.

\section{5. "Exact" Variation of Gas Properties}

Accounting for the exact variation of gas properties in combustion calculations increases the computational effor significantly, since these properies vary not only with temperature, but also with the mixture composition. For this reason, it is quite common in the combustion community to resort to simplified correlations for property variation, such as $\rho \mu=$ const., $\operatorname{Pr}=$ const., $S c=$ const., and $c_{p}=$ const. The accuracy of these correlations is very questionable in the presence of large temperature gradients, and, especially for gas mixtures the components of which differ significantly from each other in terms of molecular weight - as in the case of hydrocarbon fuels evaporating and burning in air. In spite of this fact most investigators of convective droplet evaporation and burning have also used similar approximations in their calculations, as seen in Table 6-1, obviously due to computational 
restrictions.

Enabling a reasonable analysis of the reacting gaseous flowfield surrounding the droplet in a minimum of computational times, the present model proves to be suitable also for a thorough examination of the errors introduced by the abovementioned simplifications for the variation of gas properties. Furthermore, the results obtained here for evaporating and burning droplets are valid for more general situations of reacting boundary layer flows with nonzero pressure gradients.

One significant result of this analysis is that, several of the gas properties widely assumed to be constant in combustion calculations, such as the specific heat, and Prandtl and Schmidt numbers of the gas mixture, may vary by factors of up to 3 or 4 within the viscous region near the surface containing the flame. Under these circumstances, it may become an important question what average value to choose for these properties so that a good estimate is obtained for surface transport rates as well as flame characteristics. Calculations show that this is indeed a difficult task since. in general, an average value chosen for, say, $c_{p}$ that gives a good estimate for evaporation rates, will not be as succesful when it comes to predicting flame temperature and fiame distance from the surface. In general, as suggested by Law and Williams (1972), the thermal conductivity and the specific heat capacity of the gas mixture in the region between the droplet surface and the flame turn out to be the crucial parameters for evaporation rate predictions.

Finally, it is found that results obtained from the droplet model develcped in the present work are in good agreement with experimental observations; paricularly with Law and Williams (1972) for evaporation rates from buming droplets, given that the latter is evaluated at the free-stream conditions under which the respective experiments were conducted. 


\section{REFERENCES}

Agoston, G.A., Wood, B.J., Wise, H. (1958): "Influence of pressure on the combustion of liquid spheres", Jet Propulsion 28, pp. 181-188.

Batchelor (1967): An Introduction to Fluid Mechanics, University Press, Cambridge, England.

Bellan, J., Summerfield, M. (1978): "Theoretical examination of assumptions commonly used for the gas phase surrounding a burning droplet", Combustion and Flame 33, pp. 107-122.

Brignell, A.S. (1975): "Solute extraction from an internally circulating spherical liquid drop", International Jok'snal of Heat and Mass Transfer 18, pp. 61-68.

Burke, S.P., Schumann, T.E.W. (1928): "Diffusion flames", Industrial and Engineering Chemistry 20, pp. 998-1004.

Chen, T.N., Toong, T.Y. (1964): "Laminar boundary-layer wedge flows with evaporation and combustion" in AIAA, Progress in Astronautics and Aeronautics, vol. 15, pp. 3-26.

Chiang, C.H., Raju, M.S., Sirignano, W.A. (1992): "Numerical analysis of convecting, vapotizing fuel droplet with variable properties", International Journal of Heat and Mass Transfer 35, pp. 1307-1324.

Clift, R., Grace, J.R., Weber, M.E. (1978): Bubbles, Drops and Particles, Academic Press, New York, New York.

Dwyer, H.A. (1989): "Calculations of droplet dynamics in high temperature environments", Progress in Energy and Combustion Science 15, pp. 131-158.

Dwyer, H.A., Kee, R.J., Barr, P.K., Sanders, B.R. (1984): "Transient droplet heating at high Peclet number", Journal of Fluids Engineering 105, pp. 83-88.

Dwyer, H.A., Sanders, B.R. (1984): "Detailed computation of unsteady droplet dynamics", Twentieth Symposium (International) on Combustion, pp. 1743-1749.

Dwyer, H.A., Sanders, B.R. (1S86): "A detailed study of buming fuel droplets". Twenty-first Symposium (International) on Combustion, pp. 633-639.

Dwyer, H.A., Sanders, B.R. (1988): "Unsteady influences in droplet dynamics and combustion", Combustion Scicnce and Technology 58, pp. 253-265.

Edwands, D.K., Denny, V.E., Mills, A.F. (1979): Transfer Processes, Second Edition, Hemisphere Publishing Co., Washington, D.C.

Eisenklam, P. (1961): "Atomization of liquid fuel for combustion". Journal of the Institute of Fuel 34, PP. 130-143. 
Eisenklam, P., Arunchalam, S.A., Weston, J.A. (1966): "Evaporation rates anci drag resistance of buming drops", Eleventh Symposium (Iniernational) on Combustion, pp. 715-728.

Faeth, G.M. (1977): "Current status of droplet and liquid combustion", Progress in Energy and Combustion Science 3, pp. 191-224.

Fendell, F.E. (1965): "Ignition and extinction in combustion of initially unmixed reactants", Journal of Fluid Mechanics 21, pp. 281-303.

Fernandez-Pello, A.C., Law, C.K. (1982): "On the mixed-convective flame structure in the stagnation point of a fuel particle", Nineteenth Symposium (International) on Combustion, pp. 1037-1044.

Friedlander, S.K., Keller, K.H. (1963): "The structure of the zone of diffusion controlled reaction", Chemical Engineering Science 18, pp. 365-375.

Froessling, N. (1938): "Ueber die Verdunstung fallender Tropfen", Gerlands Beitraege zur Geophysik 52, pp. 170-217.

$=\quad$ Gokalp, I., Chauveau, C., Richard, J.R., Kramer, M. L Leuckel, W. (1988): "Observations on the low temperature vaporization and envelope or wake flame buming of $n$ heptane droplets at reduced gravity during parabolic flights", Twenty-Second Symposium (International) on Combustion, pp. 2027-2035.

Gollahalli, S.R., Brzustowski, T.A. (1972): "Experimental studies on the fiame structure in the wake of a burning droplet". Fourleenth Symposium (International) on Combustion, pp. 1333-1344.

Harper, J.F., Moore, D.W. (1968): "The motion of a spherical liquid drop at high Reynolds number", Journal of Fluid Mechanics 32, pp. 367-391.

Haywood, R.J., Nafziger, R., Renksizbulut, M. (1989): "A detailed examination of gas and liquid phase transient processes in convective droplet evaporation", Journal of Heat Transfer 111, pp. 495-502.

Hirschfelder, J.O., Curtiss, C.F., Bird, R.B. (1954): Molecular Theory of Gases and Liquids, John Wiley and Sons, New York, New York.

Huang, L.J., Ayyaswamy, P.S. (1990): "Evaporation of a moving liquid droplet: solutions for intermediate Reynolds numbers". International Communications in Heat and Mass Transfer 17, pp. 27-38.

Hubbard, G.L., Denny, V.E., Mills, A.F. (1975): "Droplet cvaporation: effects of transients and variable properties", International Journal of Heai and Mass Transfer 18, pp. 1003-1008.

Jain, V.K., Mukunda, H.S. (1969): "The extinction problem in an opposed jet diffusion flame with competitive reactions", Combustion Science and Technology 1, pp. 105-117. 
Johns, L.E., Beckmann, R.B. (1966): "Mechanism of dispersed-phase mass transfer in viscous, single-drop extraction systems", AIChE Journal 12, pp. 10-16.

Kassoy, D.R., Williams, F.A. (1968): "Effects of chemical kinetics on near equilibrium combustion in nonpremixed systems", Physics of Fluids 11, Pp. 1343-1251.

Kashiwagi, T., Summerfield, M. (1972): "Ignition and flame spreading over a solid fuel: non-similar theory for a hot oxidizing boundary layer", Fourteenth Symposium (International) on Combustion, pp. 1235-1247.

Krishnamurty, L. (1976): "On gas phase ignition of a diffusion flame in the stagnation point boundary layer", Acta Astronautica 3, pp. 935-942.

Kronig, R., Brink, J.C. (1951): "On the theory of extraction from falling droplets", Applied Scientific Research A2, pp. 142-154.

Kuo, K.K. (1986): Principles of Combustion, John Wiley and Sons, New York, New York.

Lara-Urbaneja, P., Sirignano, W.A. (1981): "Theory of transient multicomponent droplet vaporization in a convective field", Eighteenth Symposium (International) on Combustion, pp. 1365-1374.

Law, C.K. (1975): "Asymptotic theory for ignition and extinction in droplet burning". Combustion and Flame 24, pp. 89-98.

Law, C.K. (1978): "Theory of thermal ignition in fuel droplet burning", Combustion and Flame 31, pp. 285-296.

Law, C.K. (1982): "Recent advances in droplet vaporization and combustion", Progress in Energy and Combustion Science 8, pp. 171-201.

Law, C.K., Chung, S.H. (1980): "An ignition criterion for droplets in sprays". Combustion Science and Technology 22, pp. 17-26.

Law, C.K., Williams, F.A. (1972): "Kinetics and convection in the combustion of alkane droplets", Combustion and Flame 19, pp. 393-405.

LeClair, B.P., Hamielec, A.E., Pruppacher, H.R., Hall, W.D. (1972): "A theoretical and experimental study of the intemal circulation in water drops falling at terminal velocity in air". Journal of the Atmospheric Sciences 29, pp. 728-740.

Lees, L. (1956): "Laminar heat transfer over blunt-nosed bodies at hypersonic flight speeds", Jet Propulsion 26, pp. 259-269.

Linan, A. (1974): "The asymptotic structure of counterfiow diffusion flames for large activation energies", Acta Astronautica 1, pp. 1007-1039.

Liu, T.M., Libby, P.A. (1970): "Boundary layer at a stagnation point with hydrogen injection". Combustion Science and Technology 2, pp. 131-144. 
Lorell, J., Wise, H., Carr, R.E. (1956): "Steady-state burning of a liquid droplet. II. Bipropellant flame", Journal of Chemical Physics 25, pp. 325-331.

Natarajan, R., Brzustowski, T.A. (1970): "Some new observations on the combustion of hydrocarbon droplets at elevated pressures", Combustion Science and Technology 2. pp. 259-269.

Okajima, S., Kumagai, S. (1974): "Further investigations of combustion of free droplets in a freely falling chamber including moving droplets", Fifieenth Symposium (International) on Combustion, pp. 401-407.

Pan, Y.F., Acrivos A. (1968): "Heat transfer at high Peclet number in regions of closed streamlines". International Journal of Heat and Mass Transfer 11, Pp. 439 444.

Panton, R.L. (1984): Incompressible Flow, John Wiley \& Sons, New York, New York.

Prakash, S., Sirignano, W.A. (1978): 'Liquid fuel droplet heating with internal circulation". International Journai of Heat and Mass Transfer 21, pp. 885-895.

Prakash, S., Sirignano, W.A. (1980): "Theory of convective droplet vaporization with unsteady heat transfer in the circulating liquid phase". International Journal of Heat and Mass Transfer 23, pp. 253-268.

Rangel, R.H., Femandez-Pello, A.C. (1984): "Mixed convective droplet combustion with internal circulation", Combustion Science and Technology 42, pp. 47-65.

Rangel, R.H., Fernandez-Pello, A.C. (1986): "Droplet ignition in mixed convection" in AlAA, Progress in Astronautics and Aeronautics, vol. 105, pp. 239-252.

Ranz, W.E., Marshall, W.R. (1952): "Evaporation from drops", Chemical Engincering Progress 48, pp. 141-146, 173-180.

Renksizbulut, M., Haywood, R.J. (1988): "Transient droplet evaporation with variable properities and intemal circulation at intermediate Reynolds numbers", Inicrnational Journal of Multiphase Flow' 2, pp. 189-202.

Renksizbulut, M., Yuen, M.C. (1983a): "Experimental study of droplet evaporation in a high-temperature air stream", ASME Journal of Heat Transfer 105, pp. 384-388.

Renksizbulut, M., Yuen, M.C. (1983b): "Numerical study of droplet evaporation in a high-temperature stream". ASME Journal of Heal Transfer 105. pp. 389-397.

Rivkind, V.I., Ryskin, G.M. (1976): "Flow stsucture in motion of a spherical drop in a fluid medium at intennediate Reynolds numbers", Fluid Djnamics (Iricstiya Alia. demii Nauk SSSR - Mekhanika Zhidkosti i Gaza), English iranslation, 11. pp. 5-12.

Rivkind, V.I., Ryskin, G.M., Fishbein, G.A. (1976): "Flow' around a spherical drop at intermediate Reynolds numbers". Applicd Mathematics and Mcchanics (PMMS). 
English translation, 40, pp. 687-691.

Rossini, D.R., Pitzer, K.S., Amet, R.L., Braun, R.M., Pimentel, G.C. (1953): Selected Values of Physical and Thermodynamic Properties of Hydrocarbons and Related Compounds, Carnegie Press, Pittsburgh.

Sanders, B.R., Dwyer, H.A. (1987): "Modeling unsteady droplet combustion processes", Proceedings of tine ASME/JSME Thermal Engineering Joint Conference, Volume 1, pp. 3-10.

Sirignano, W.A. (1983): "Fuel droplet vaporization and spray combustion theory", Progress in Energy and Combustion Science 9, pp. 291-322.

Spalding, D.B. (1953): "Experiments on burning and extinction of liquid fuel spheres", Fuel (London) 32, pp. 169-185.

Teodorczyk, A., Wojcicki, S. (1983): "The combustion of a liquid fuel droplet during forced convection", Preprint 1983 Spring Meeting, Western States Section of the Combustion Institute, Paper WSCI 83-36.

Tong, A.Y., Sirignano, W.A. (1982): "Analytical solution for diffusion and circulation in a vaporizing droplet", Nineteenth Symposium (International) on Combustion, pp. 1007-1020.

Van Dyke, M. (1975): Perturbation Methods in Fluid Mechanics, Annotated Edition, Parabolic Press, Stanford, California.

Wilke, C.R. (1950): "A viscosity equation for gas inixtures", Journal of Chemical Physics 18, pp. 517-519.

Williams, A. (1973): "Combustion of droplets of liquid fuel: a review", Combustion and Flame 21, pp. 1-31.

Williams, A. (1990): Combustion of Liquid Fuel Sprays, Butterworths Publishing Co., London, England.

Williams, F.A. (1971): "Theory of combustion in laminar flows", Annual Revicw of Fluid Mechanics 3, pp. 171-188.

Williams, F.A. (1985): Combustion Theory, Second Edition, Benjamin/Cummings Publishı.ng Co., Menlo Park, California.

Yuen, M.C., Chen, L.W. (1976): "On drag of evaporating liquid droplets", Combustion Science and Technology 14, pp. 147-154. 


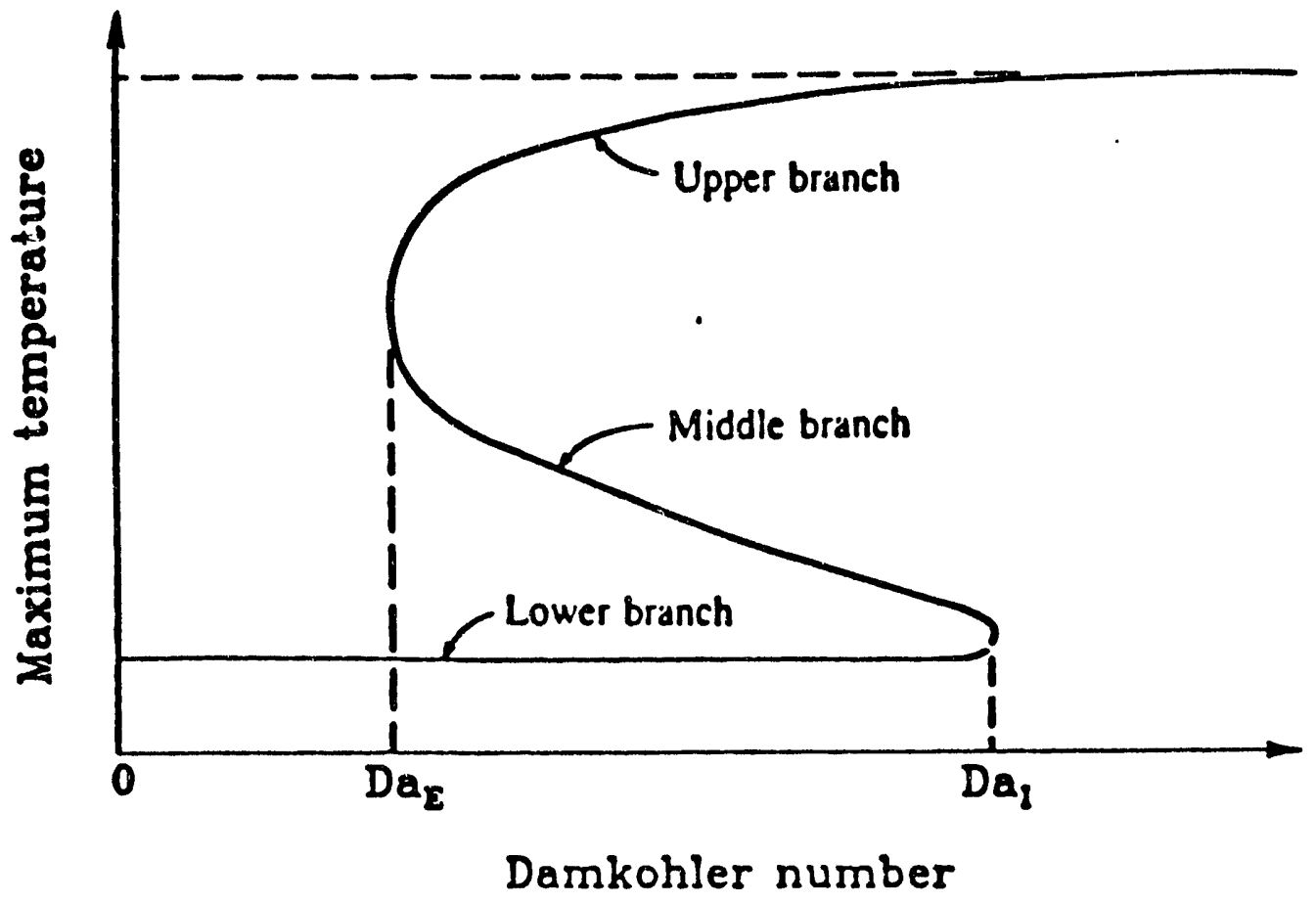

Figure 1-1. Schematic illustration of the dependence of the maxinum temperature on the relevam Damnhohler number for diffusion th:mes - after Williums (IS\$5). 


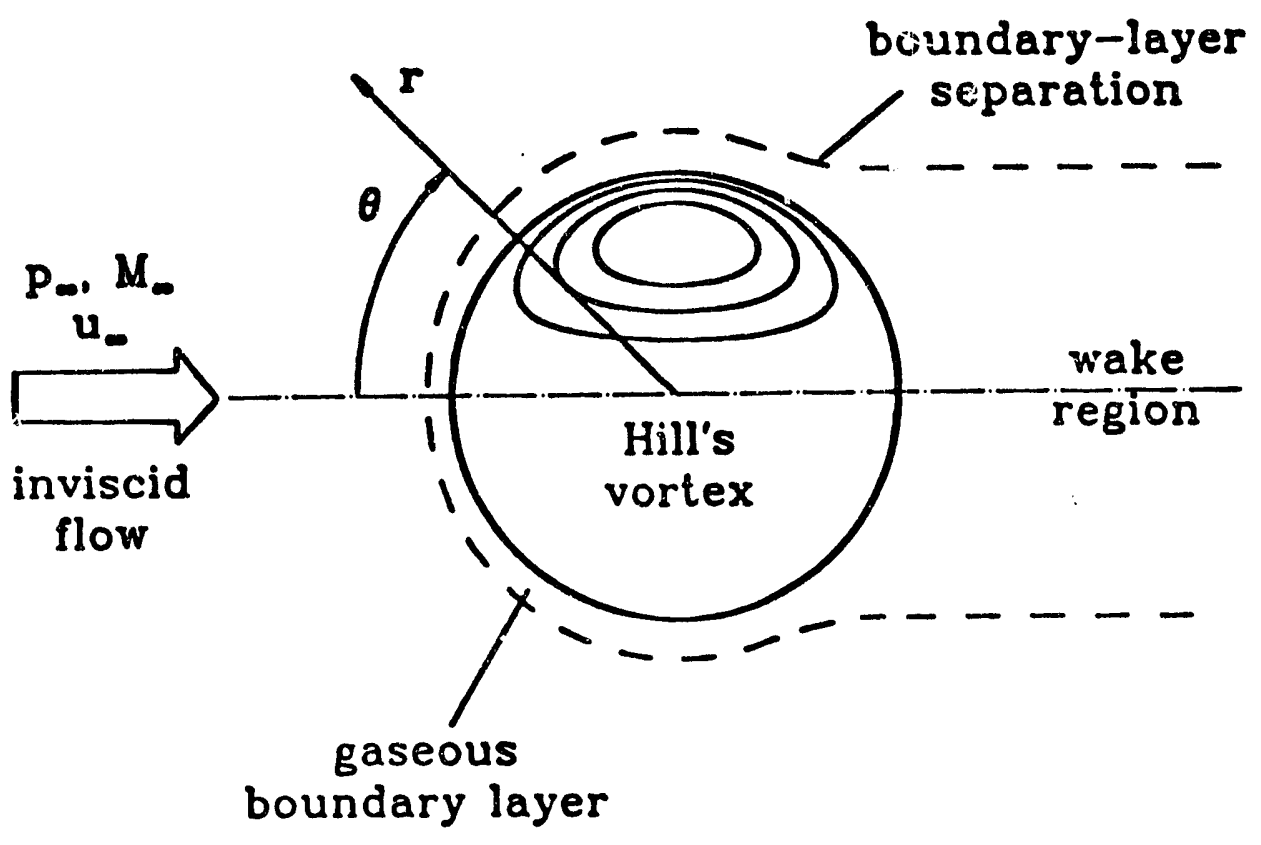

Figure 2-1. Schematic diagram of the fowfield associated with a liquid fuel droplet in a convective environment. 

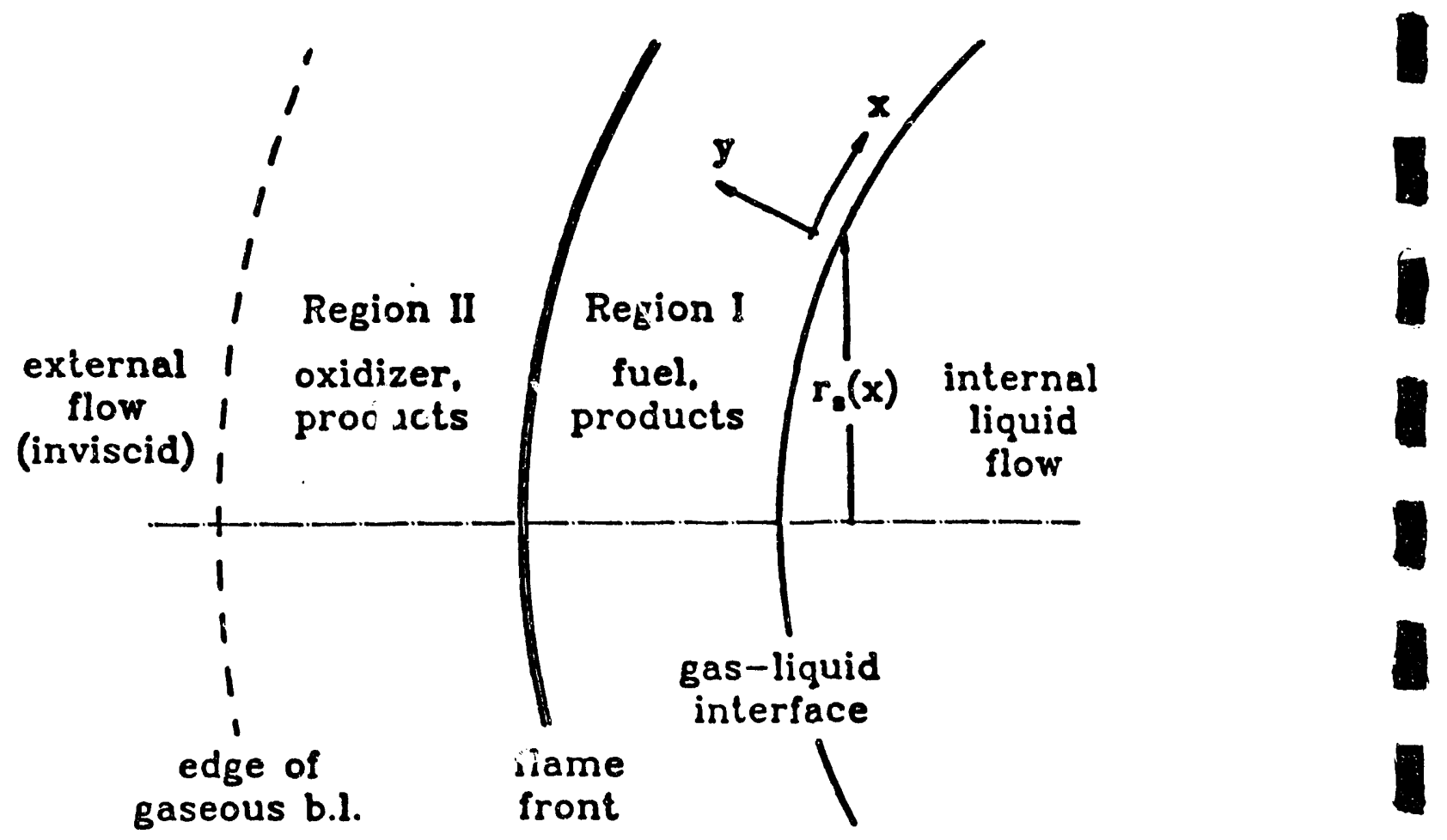

Figure 2-2. Characteristics of ille boundary layer and reaction zone near the surface of the liguid droplet. 


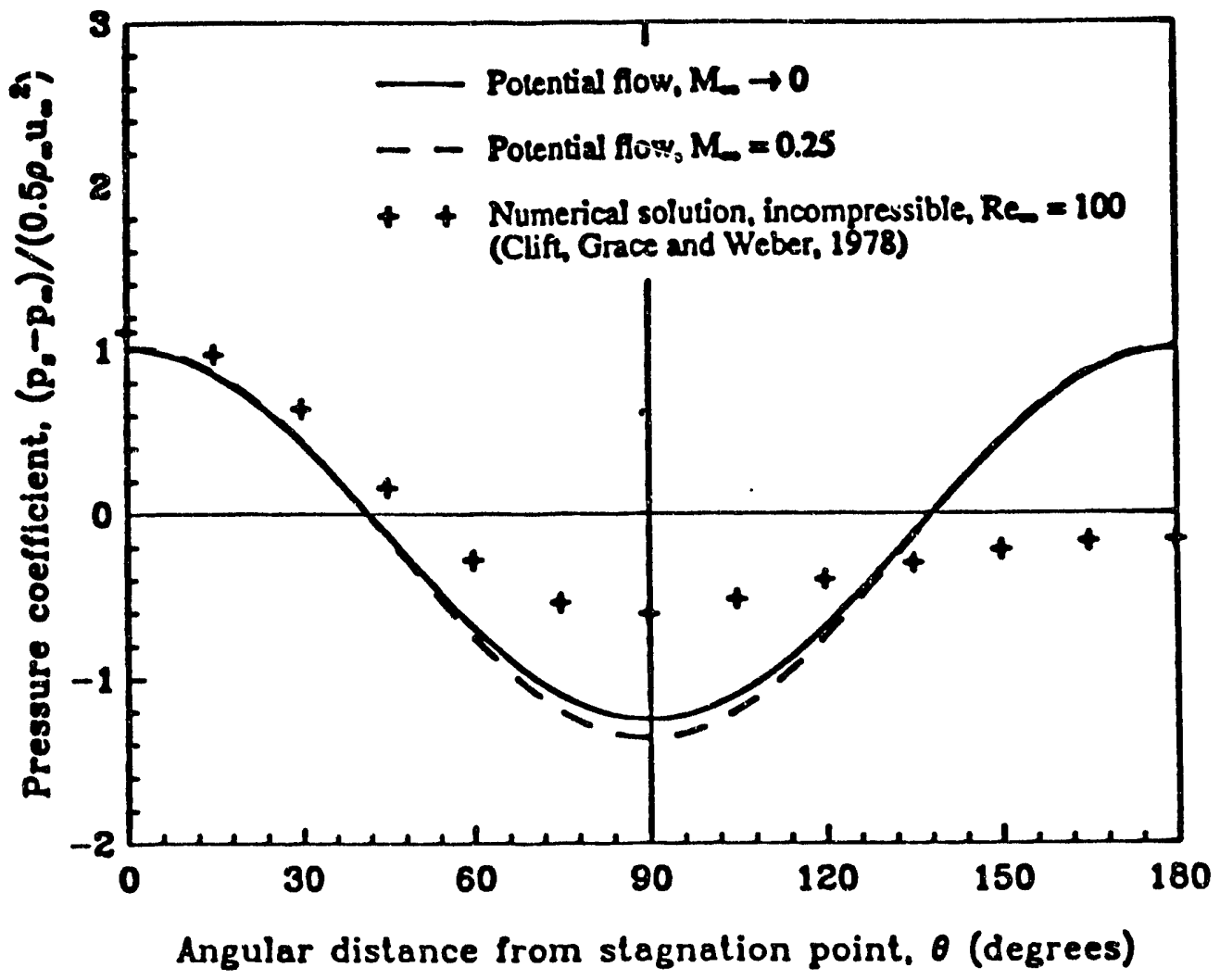

Figure 2-3. Variation of pressure distribution along the surface of a rigid sphere. 


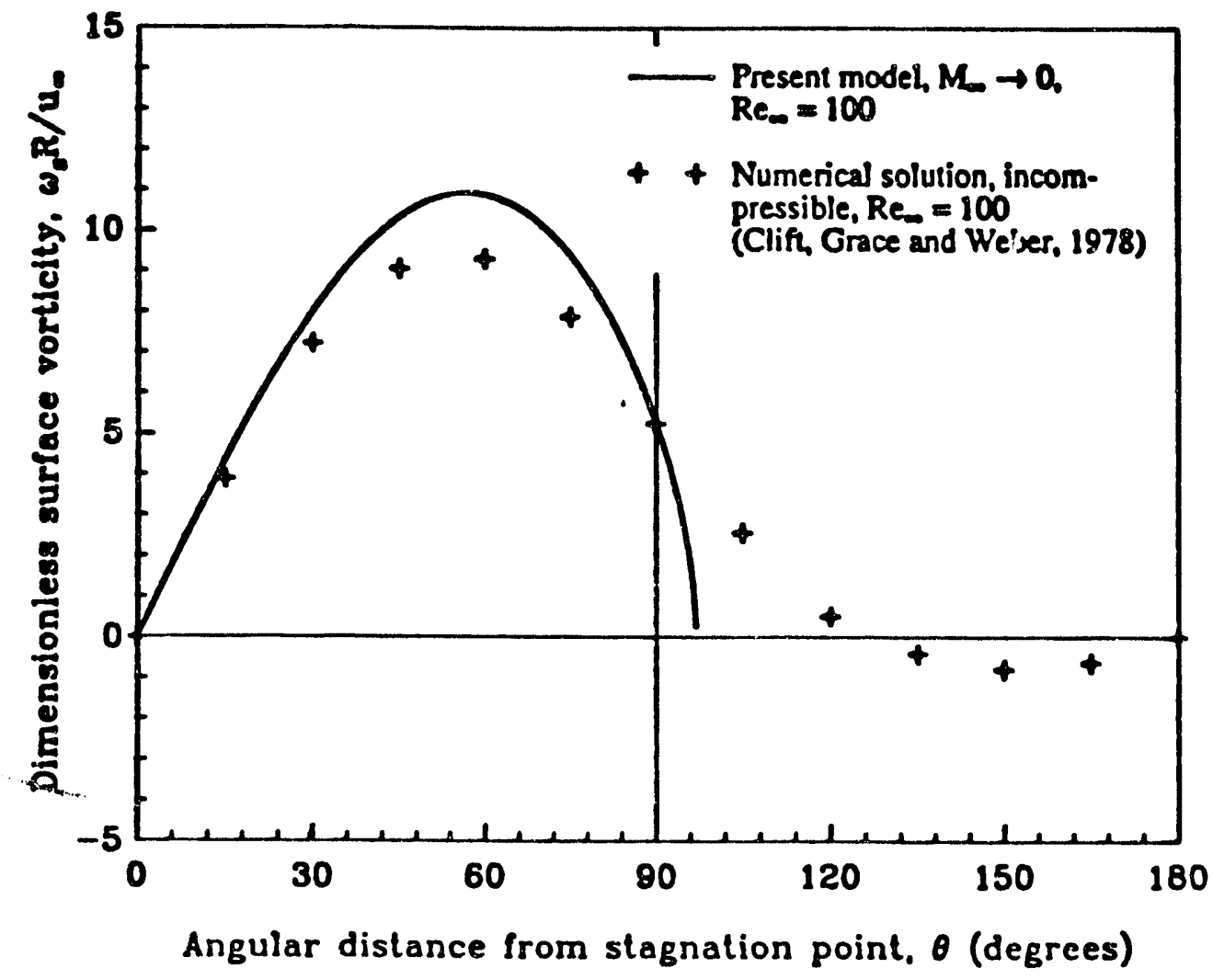

Figure 2-4. Variation of dimensionless vorticity along the surface of a rigid sphere. 


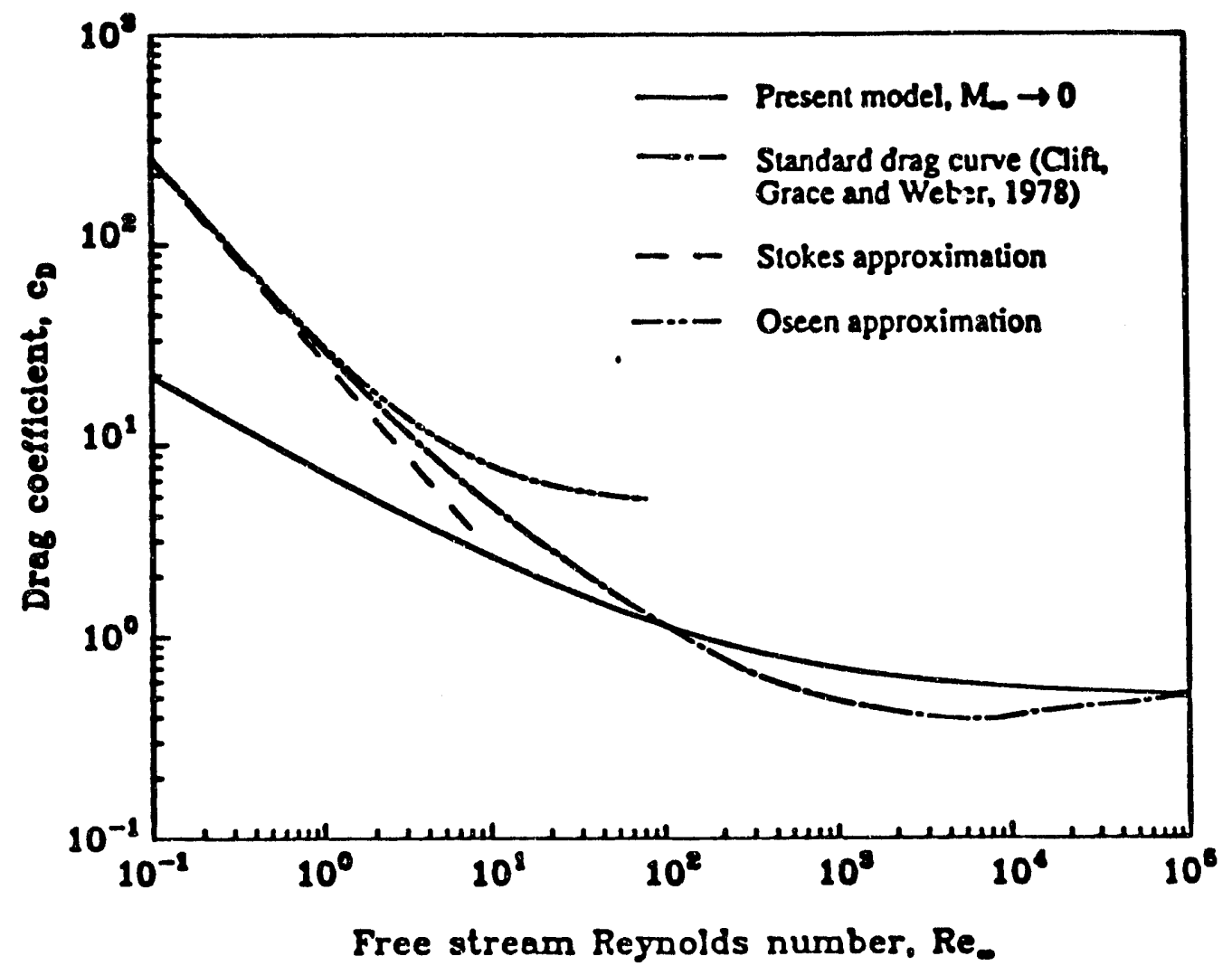

Figure 2-5. Variation of drag coefficient with free stream Reynolds number for a rigid sphere. 


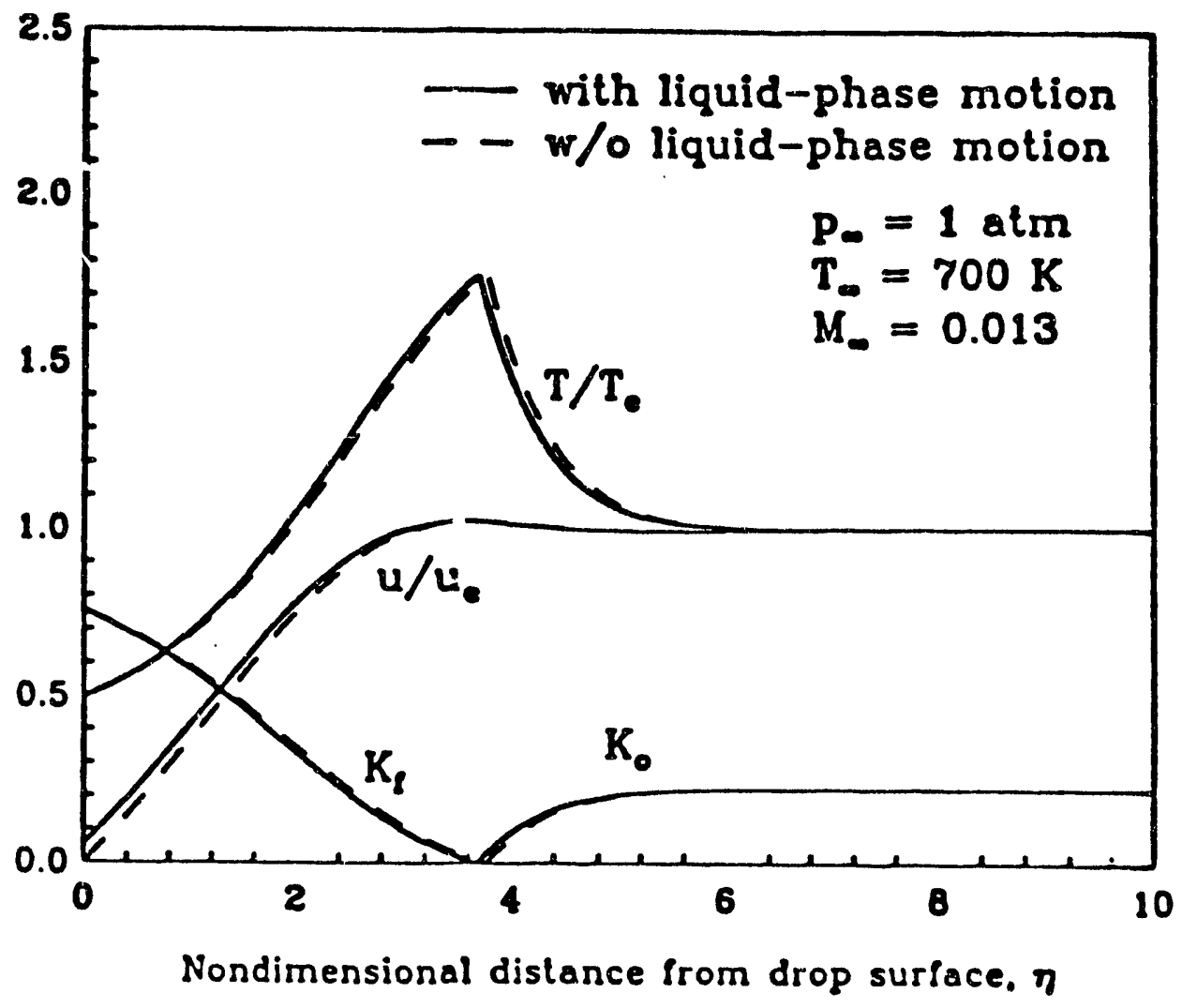

Figure 2-6. Predicted velocity, temperature, and concentration profiles in the boundary layer of the burning droplet at an angular location $\theta=60^{\circ}$ from the stagnation point. 


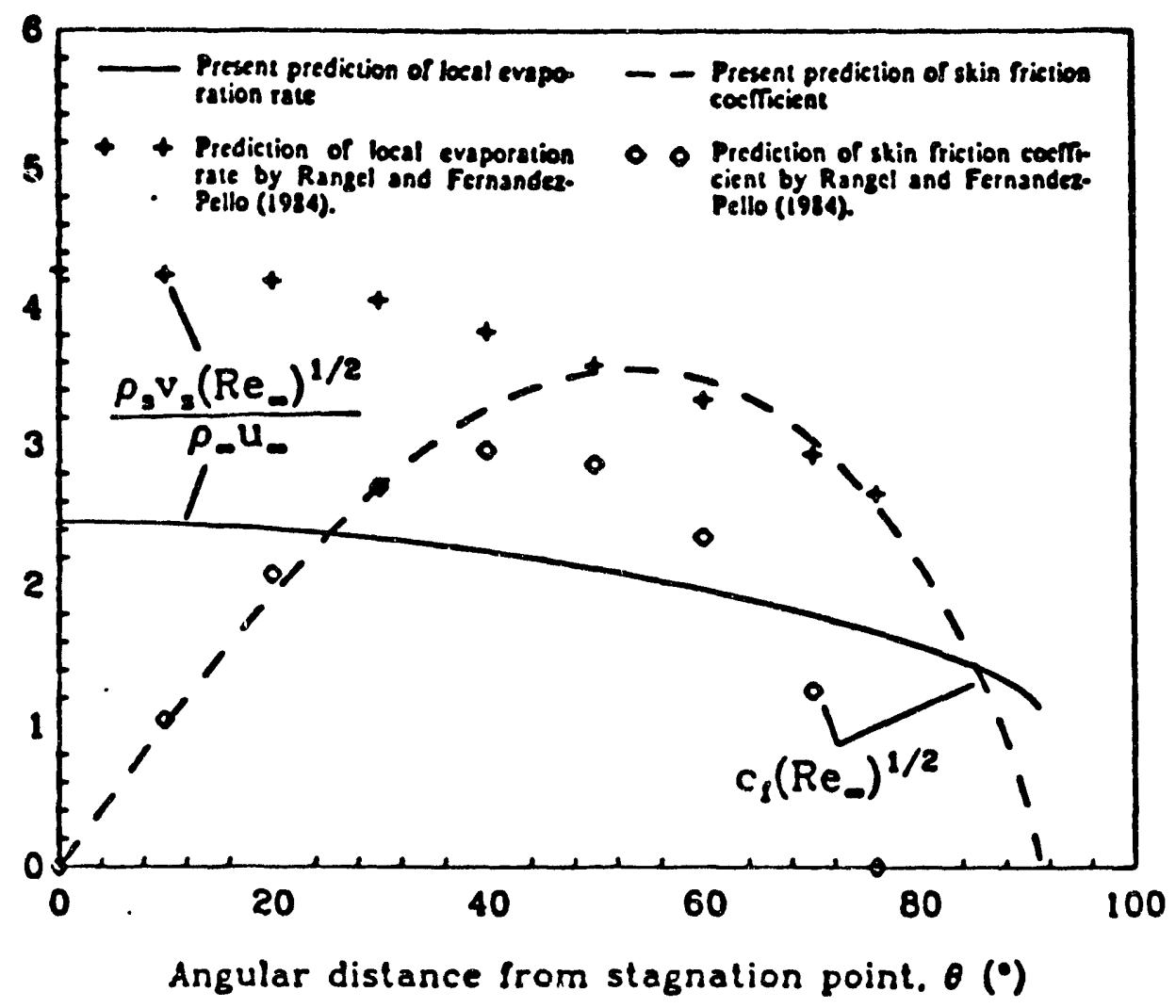

Figure 2-7. Comparison of evaporation rate and skin friction coefficient with the predictions of Rangel and Fernandez-Pello (1984) at $p_{\infty}=1$ atm, $T_{-}=293 K_{0} R \varepsilon_{-}=120, M_{\infty}=0.01$. 


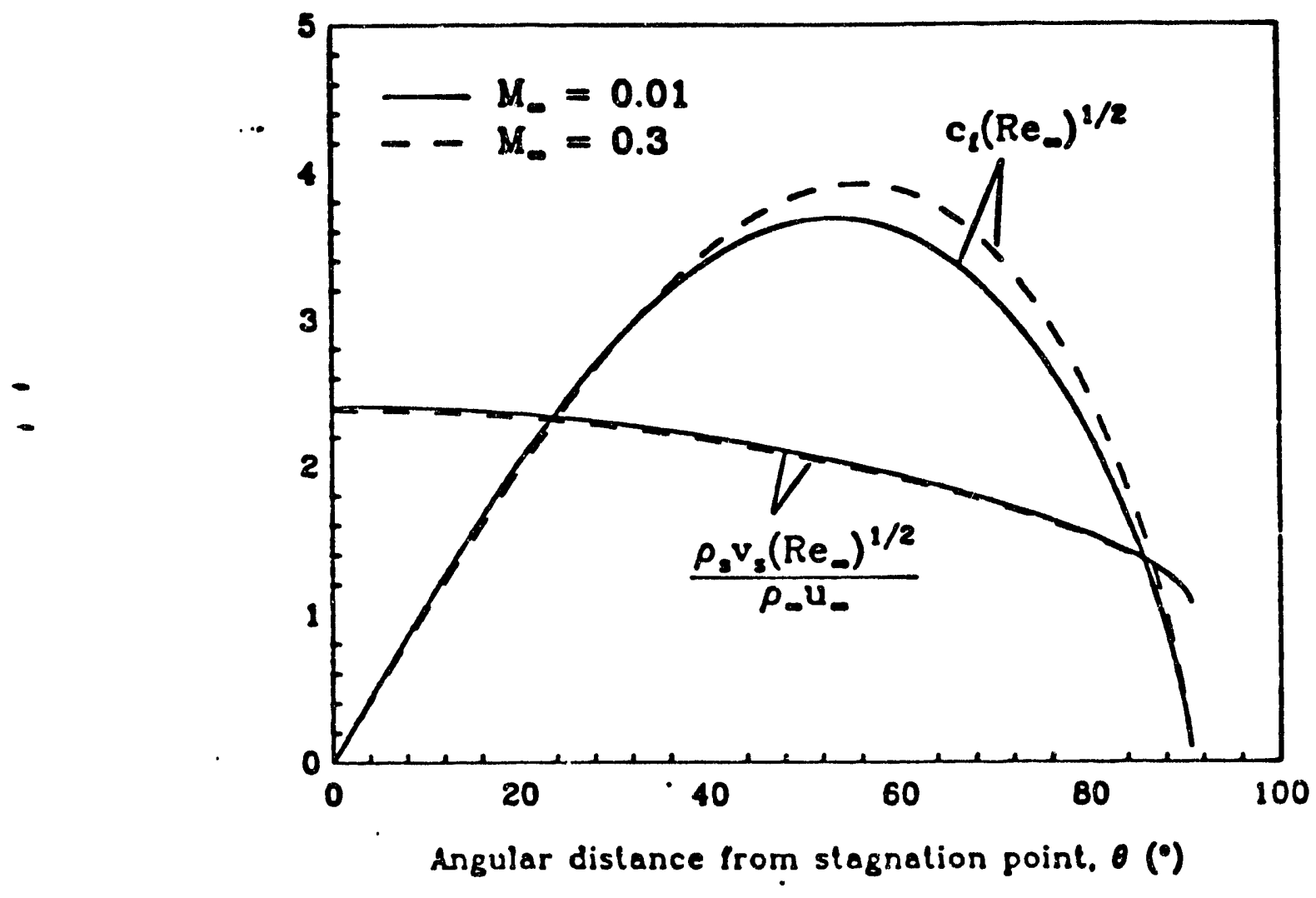

Figure 2-8. Effect of the freestream Mach number, $M_{-}$, on the skin friction coefficient and evaporation rate per unit area along the buming droplet surface at $p_{\infty}=1 \mathrm{~atm} T_{0} T_{-}=293 \mathrm{~K}, \mathrm{Re}_{\infty}=50$. 


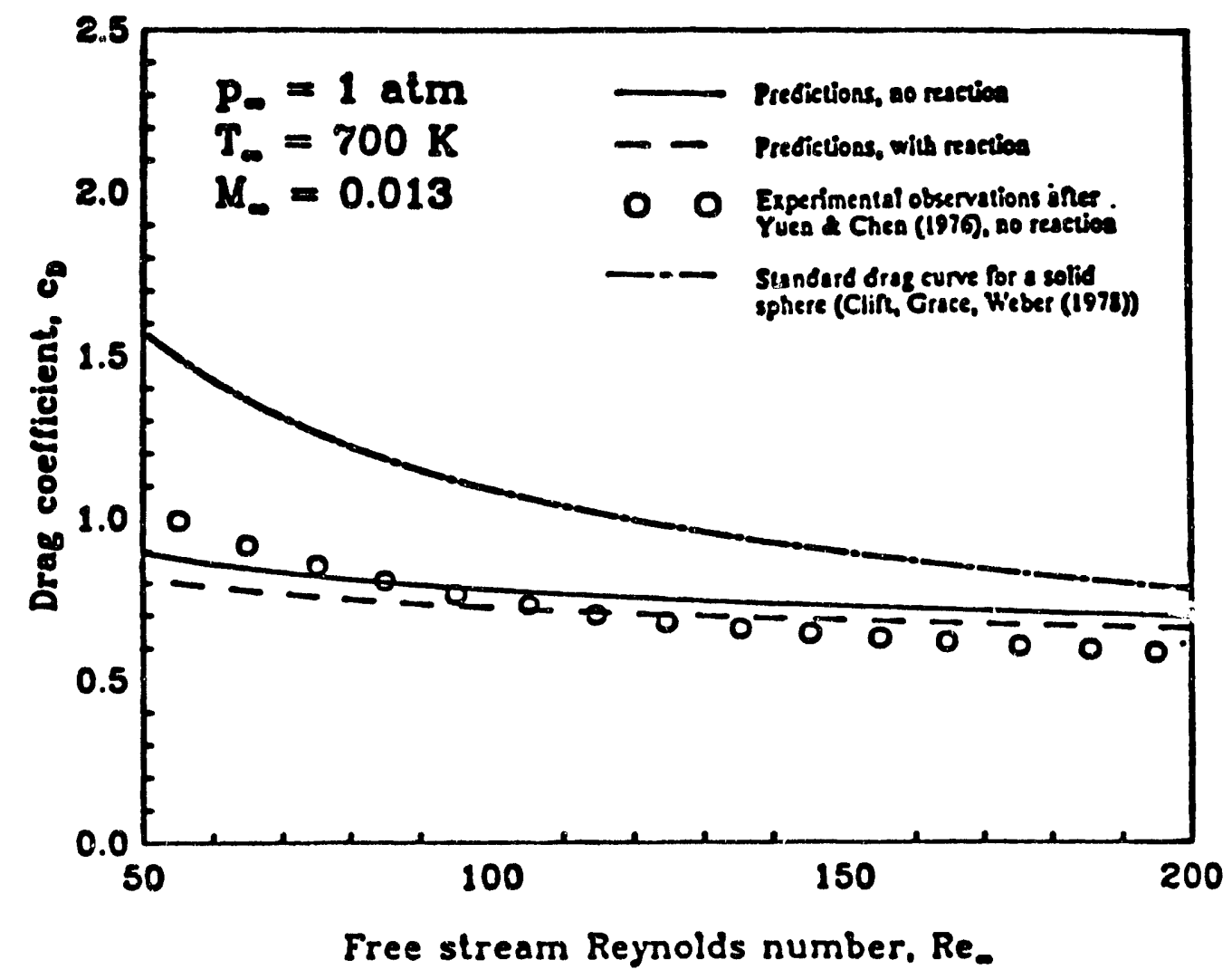

Figure 2-9. Predicted variation in drag cosfficient of the droplet with freestream Reynolds number, for both evaporating and burning droplets. Com. parison is made with the experimental data of Yuen and Chen (1976) for an evaporating droplet, and with the "standard" curve for the drag on a solid sphere. 


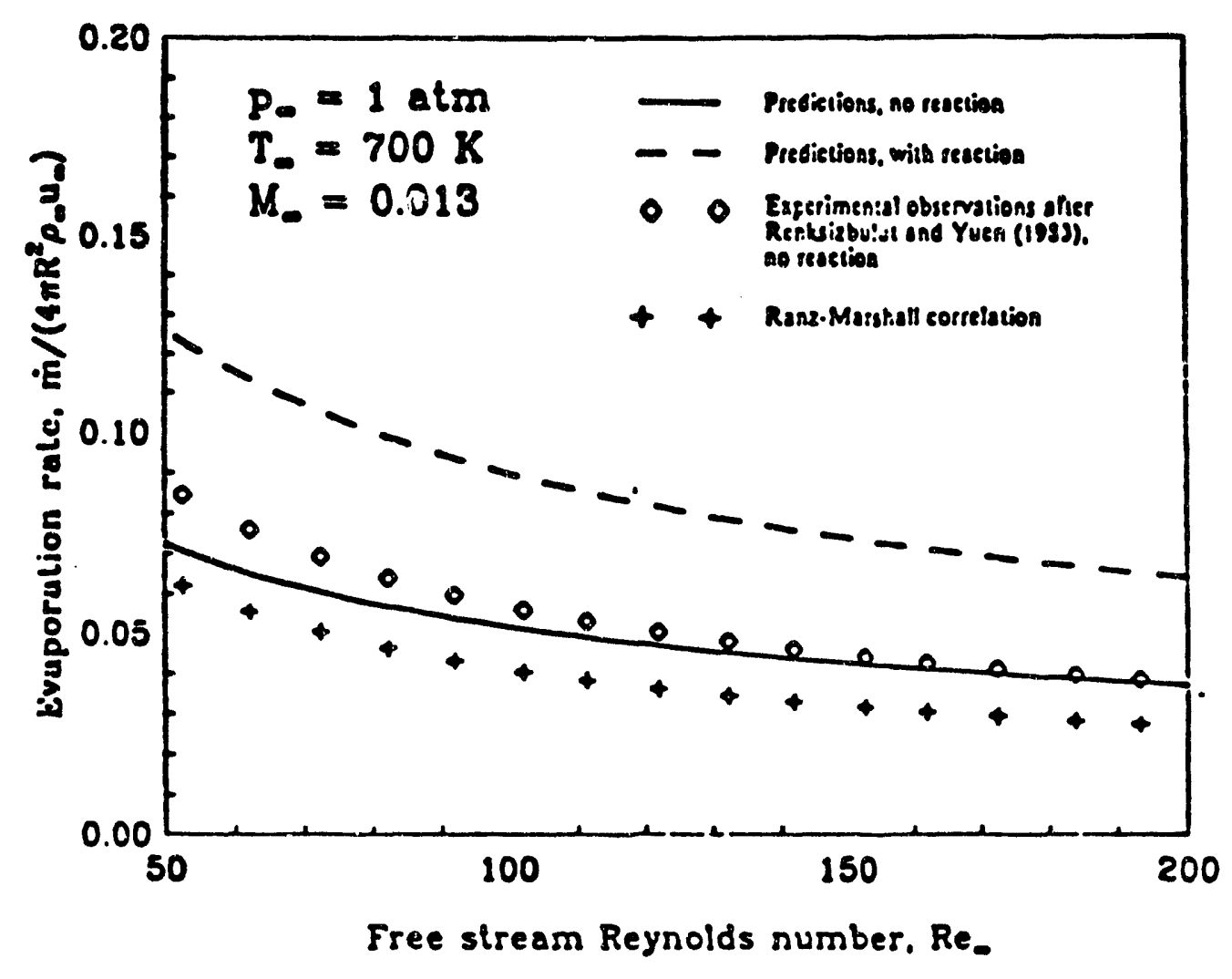

Figure 2-10. Predicted variation in mass transfer rate of the droplet with Reynolds number, for evaporating and burning droplets. Comparison is made with Renksizbulut-Yuen and Froessling/Ranz-Marshall correlations for an evaporating droplet. 


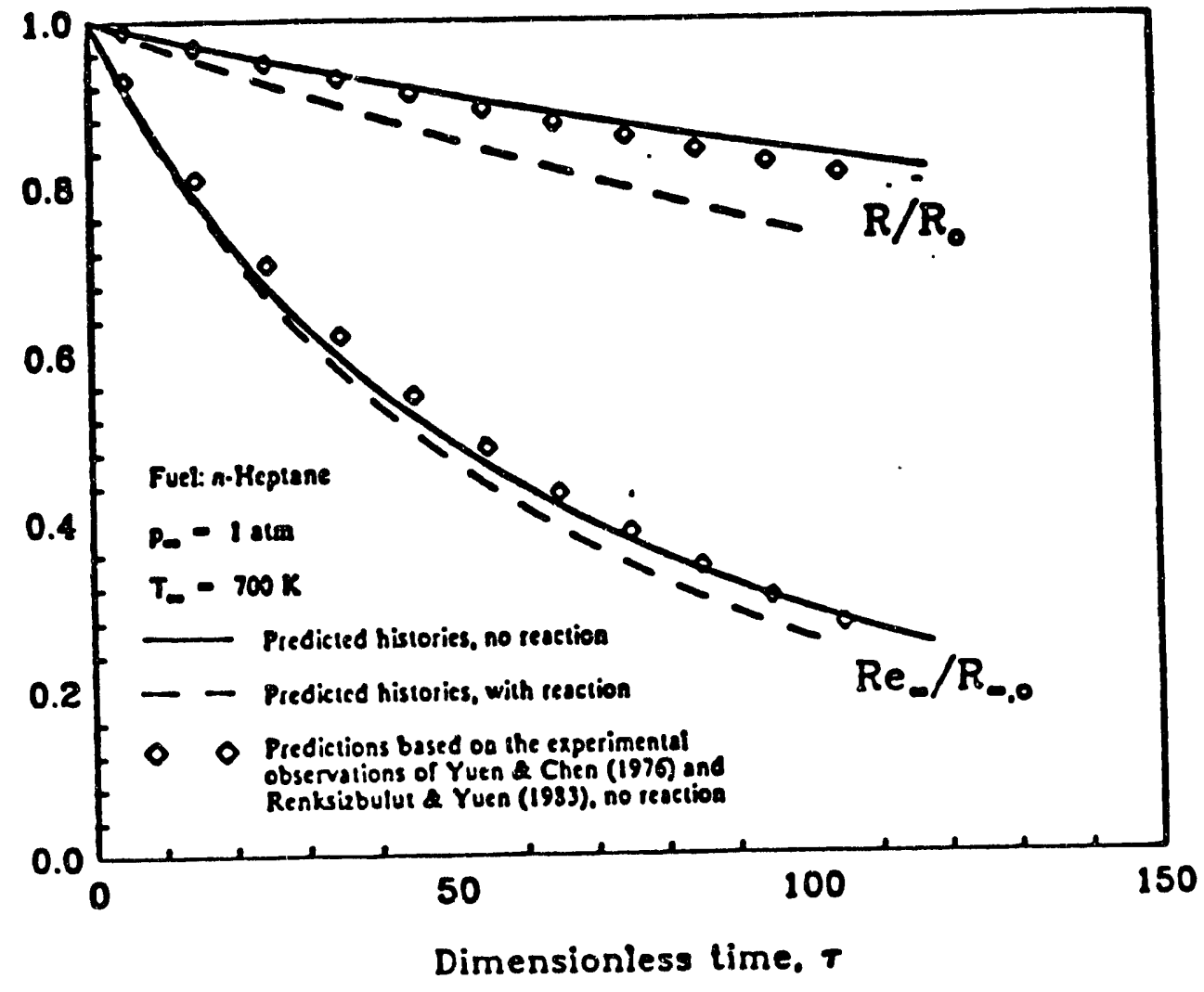

Figure 2-11. Predicted droplet histories (radius and effective Rey'nolds number). for evaporating and burning droplets. Comparison is made with the experimental data of Renksizbulut and Yuen (1983a), using the data on drag coefficient from Yuen and Chen (1976). 


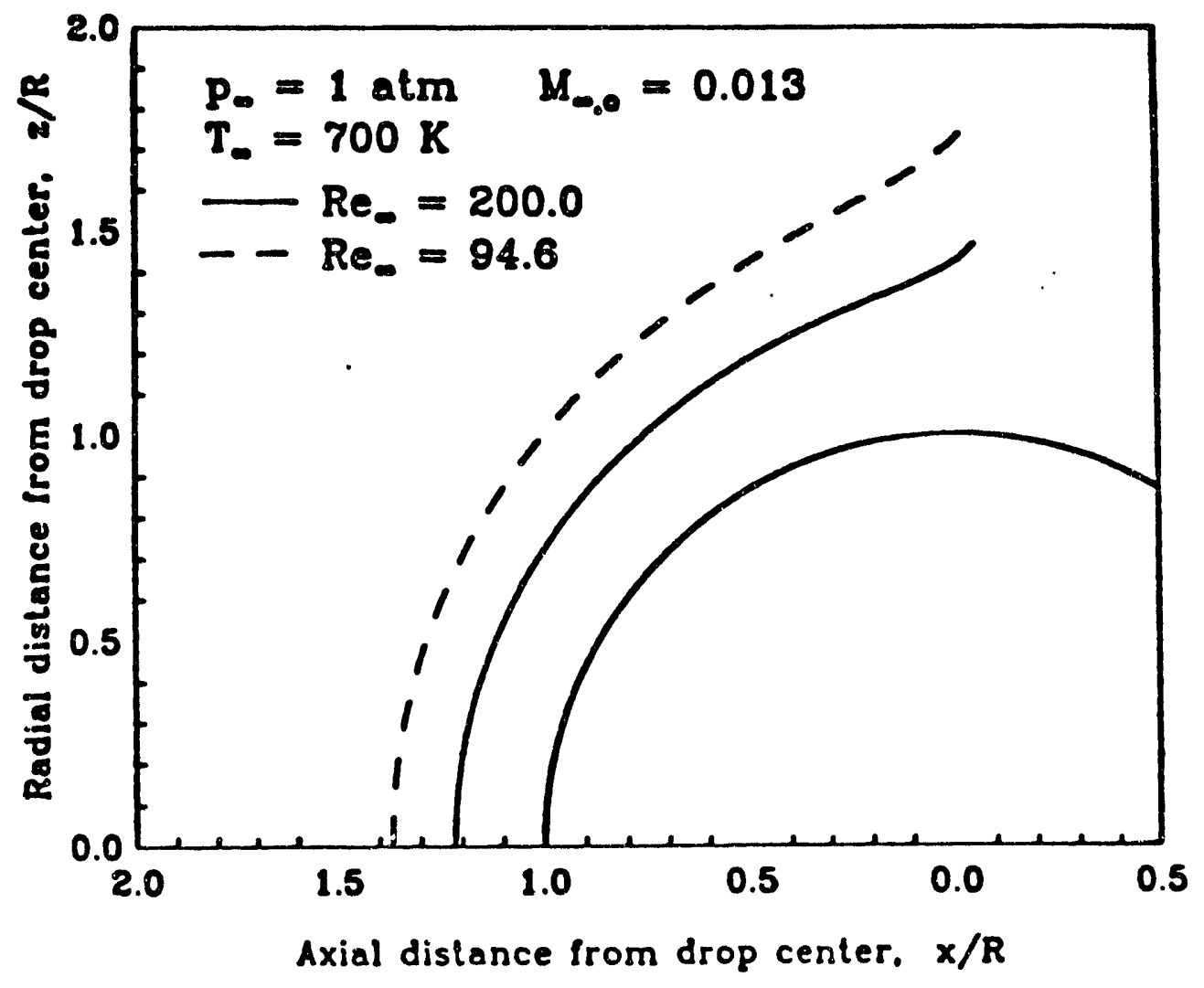

Figure 2-12. Predicted flame shapes about spherical droplet for two different Reynolds numbers. 


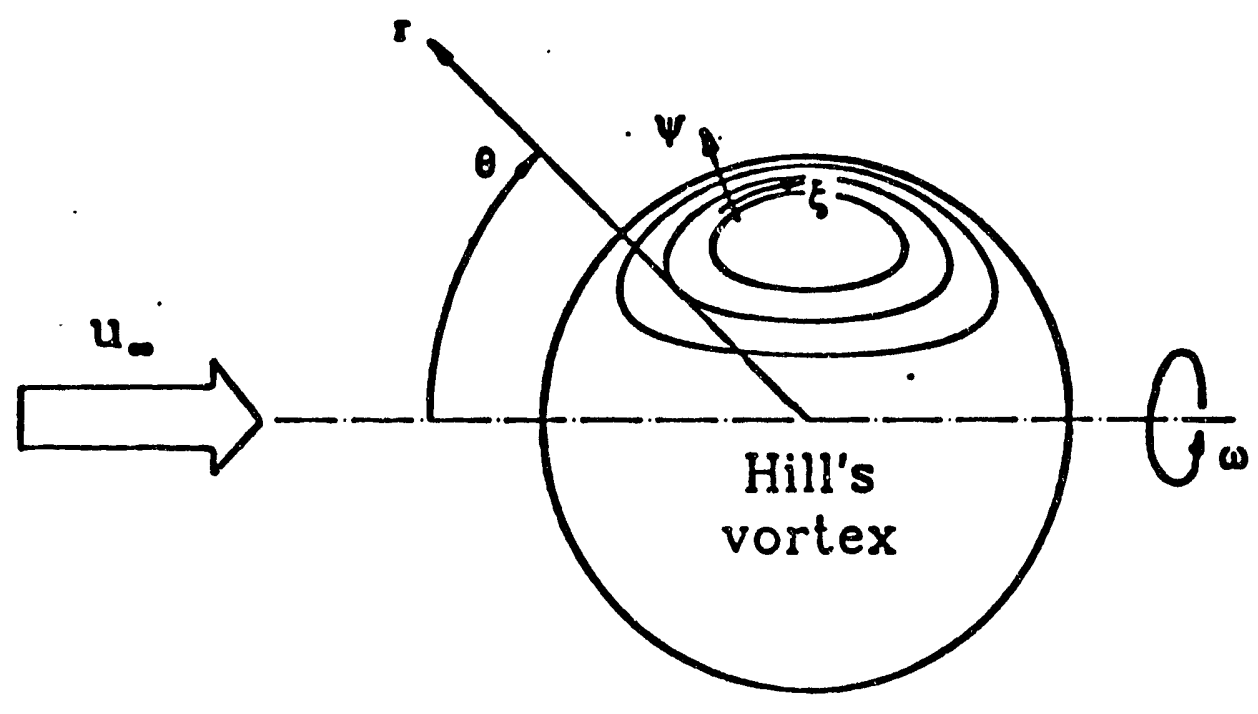

Figure 3-1. Illustration of the orthogonal streamline coordinate system $(\xi, \psi, \omega)$. 


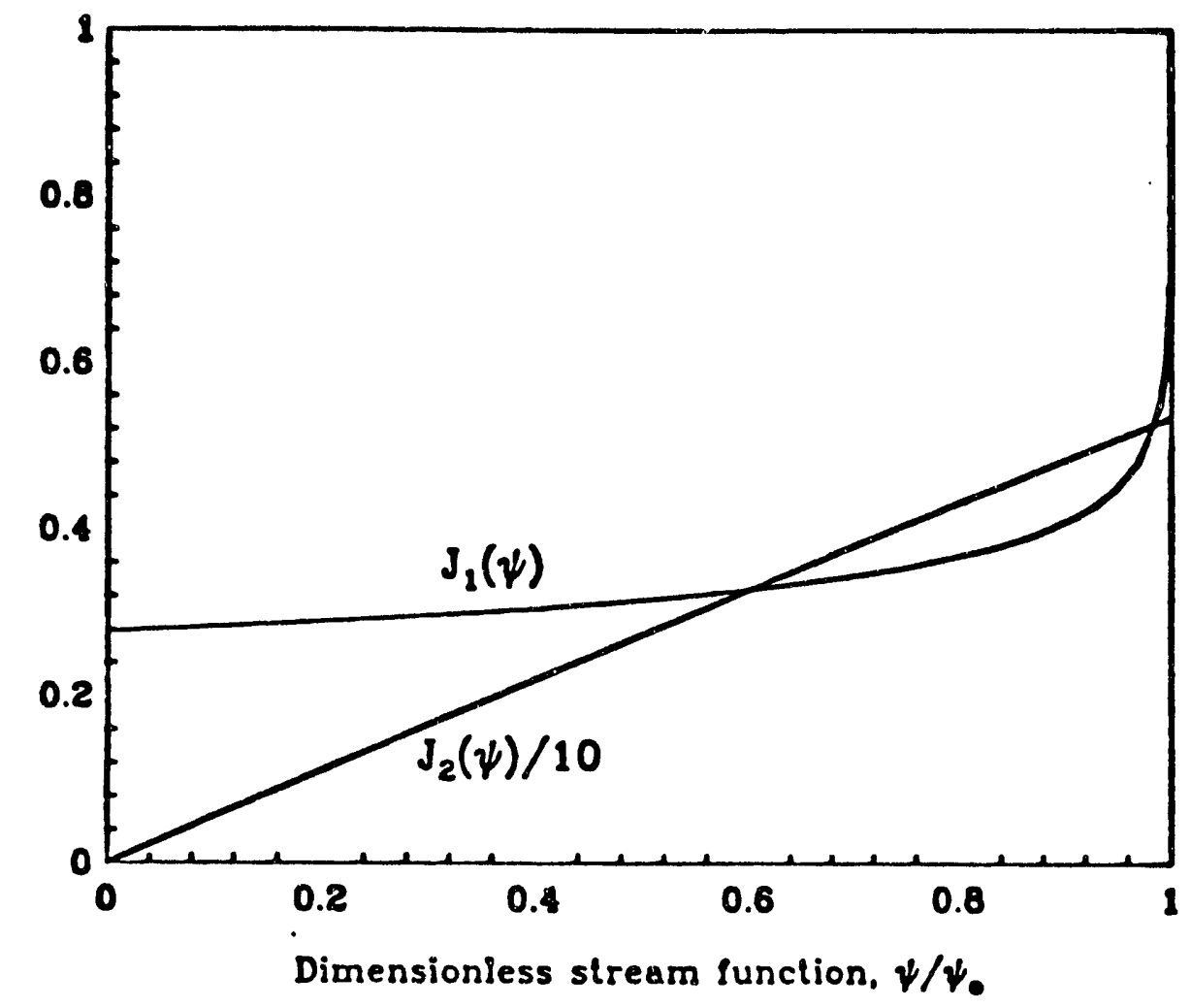

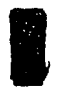
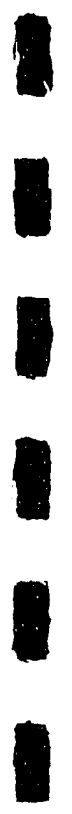

Figure 3-2. The functions $J_{1}(\psi)$ and $J_{2}(\psi)$. 


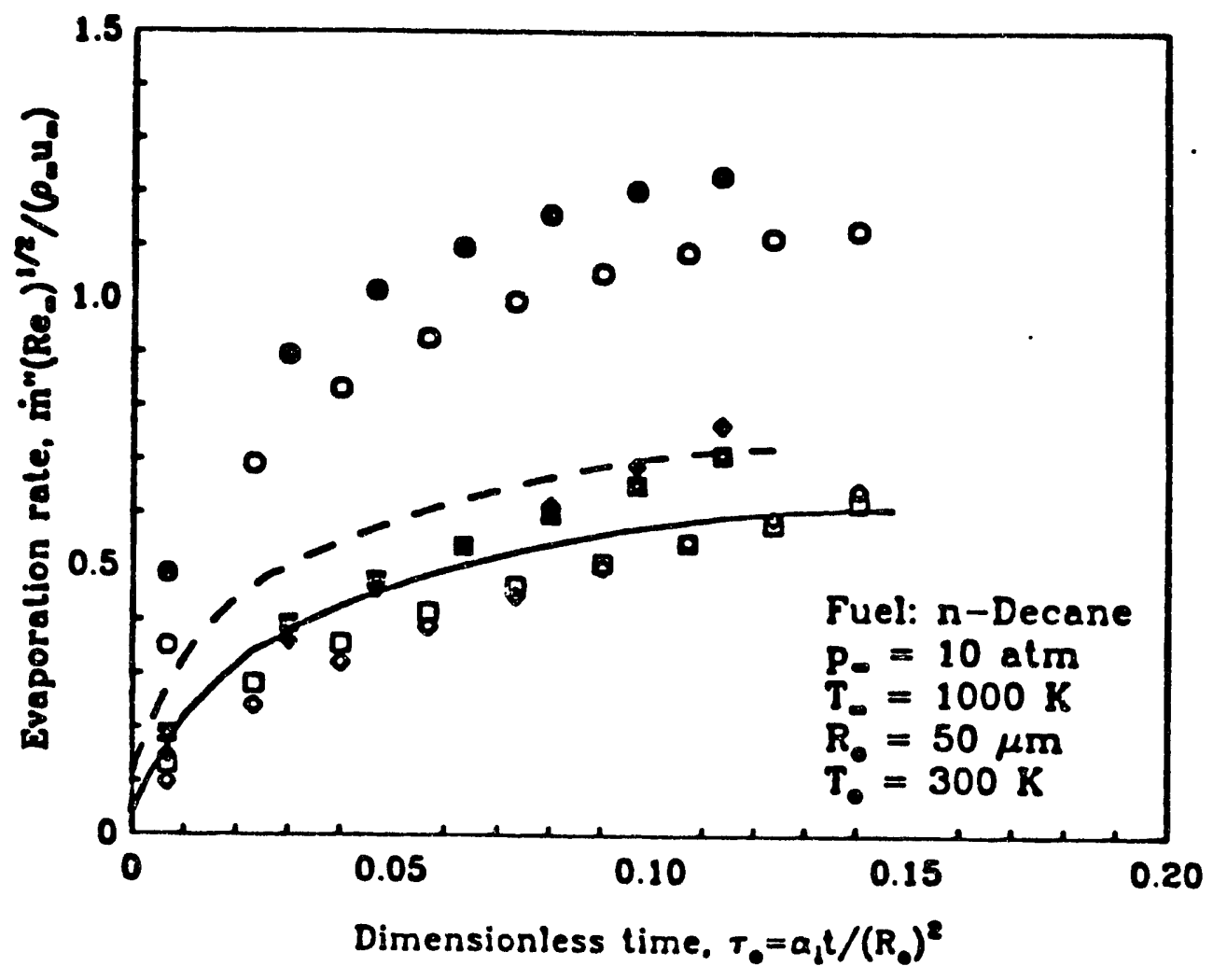

\begin{tabular}{|c|c|c|}
\hline & $\begin{array}{l}\text { without } \\
\text { reaction }\end{array}$ & $\begin{array}{c}\text { with } \\
\text { reaction }\end{array}$ \\
\hline Present model & $\longrightarrow$ & - - \\
\hline Ranz-Marshali (1952) & - 0 & $\bullet \bullet$ \\
\hline Renksizbulut-Yuen (1983) & D & 0 \\
\hline Natarajan-Brzustowski (1970) & 00 & $\oplus$ \\
\hline
\end{tabular}

Figure 3-3. Predicted temporal variation of the nondimensional evaporation rate. compared with various empirical correlations. 


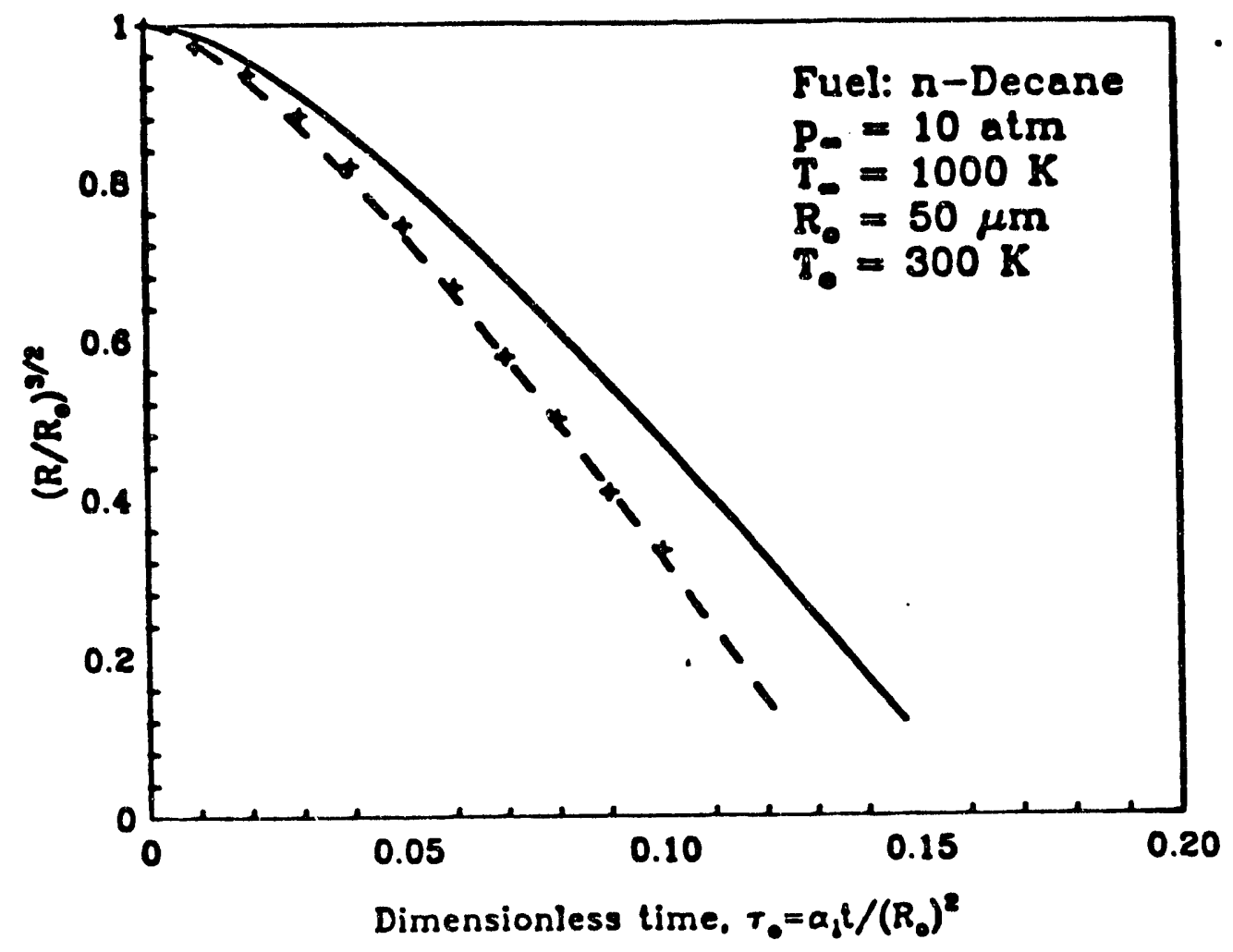

\begin{tabular}{|l|c|c|}
\cline { 2 - 3 } \multicolumn{1}{c|}{} & $\begin{array}{c}\text { without } \\
\text { reaction }\end{array}$ & $\begin{array}{c}\text { with } \\
\text { reaction }\end{array}$ \\
\hline Present model & - & -- \\
\hline Prakash-Sirignano (1980) & ++ & n/a \\
\hline
\end{tabular}

Figure 3-4. Predicted temporal change in droplet radius, compared with the predictions of Prakash and Sirignano (1980). 

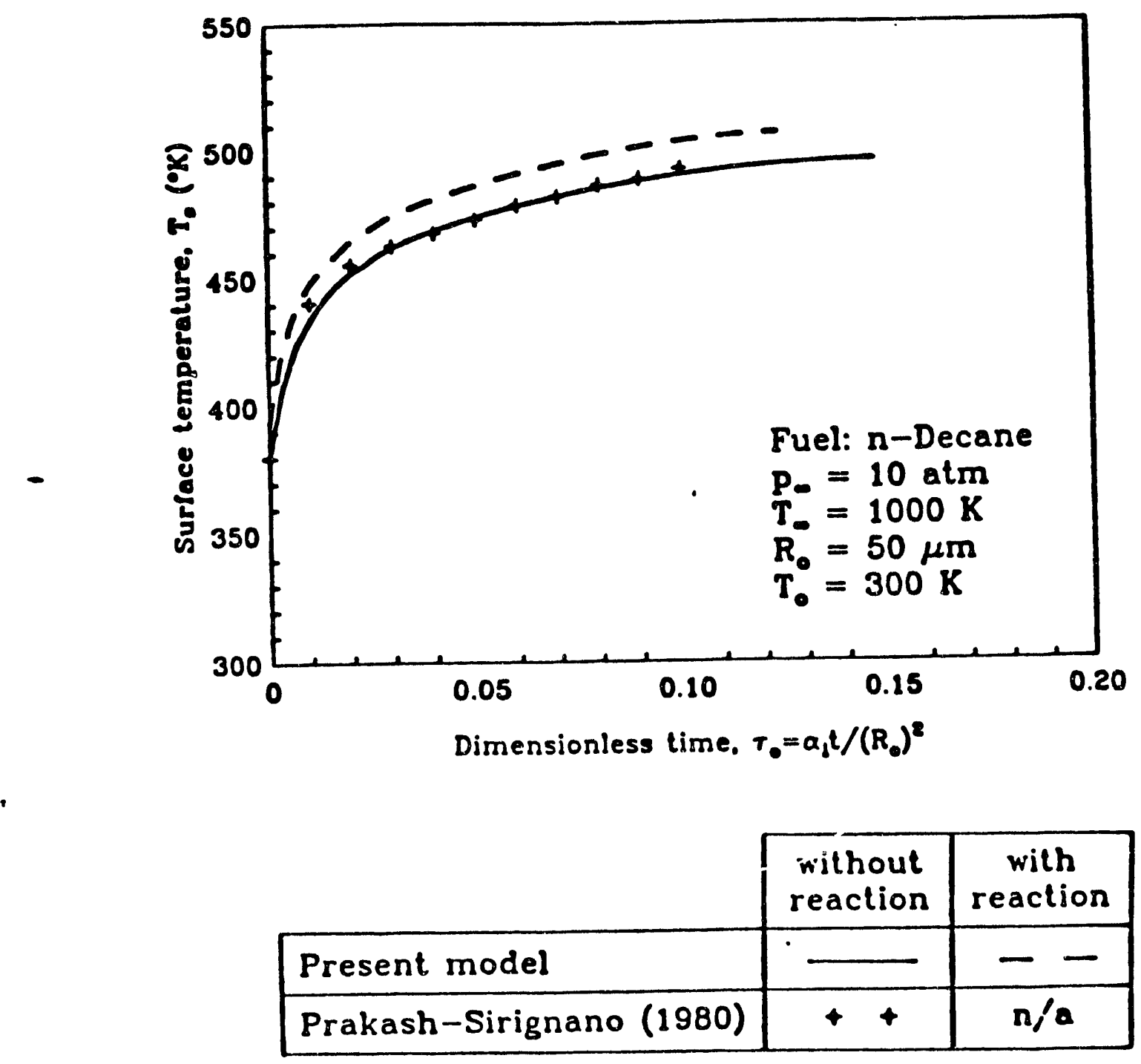

Figure 3-5. Predicted temporal change in surface temperature, compared with the predictions of Prakash and Sirignano (1980) at $\theta=90^{\circ}$. 


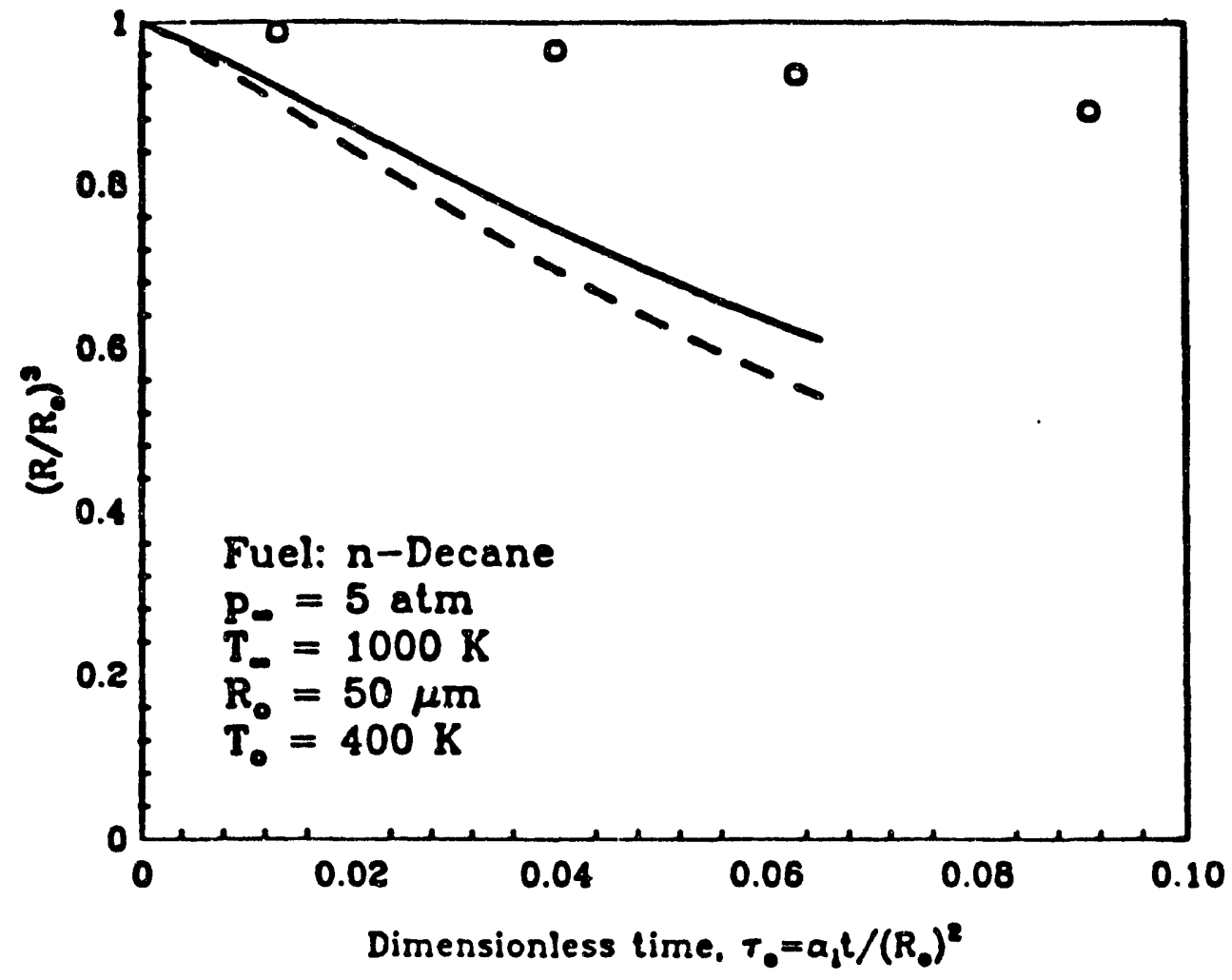

\begin{tabular}{|c|c|c|}
\hline & $\begin{array}{l}\text { without } \\
\text { reaction }\end{array}$ & $\begin{array}{l}\text { with } \\
\text { reaction }\end{array}$ \\
\hline Present model & $\longrightarrow$ & - - \\
\hline Sanders and Dwyer (1987) & 00 & $\mathrm{n} / \mathrm{a}$ \\
\hline
\end{tabular}

Figure 3-6. Predicted temporal change in droplet radius, compared with the predictions of Sanders and Dwyer (1987). 


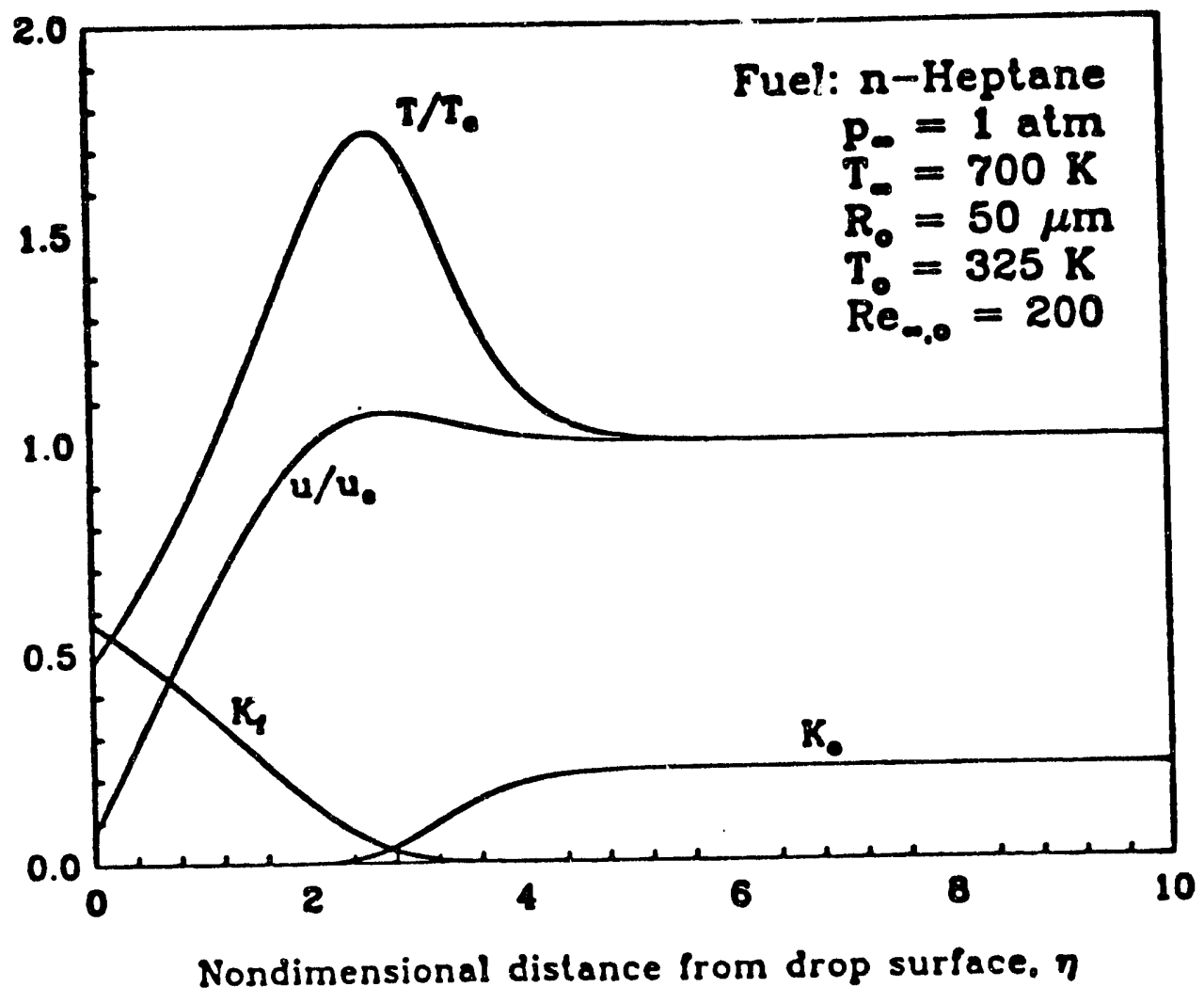

Figure 4-1. Predicted temperature, velocity, and species mass fraction profiles at an angular distance $\theta=60^{\circ}$ from the upstream stagnation point of the droplet. 


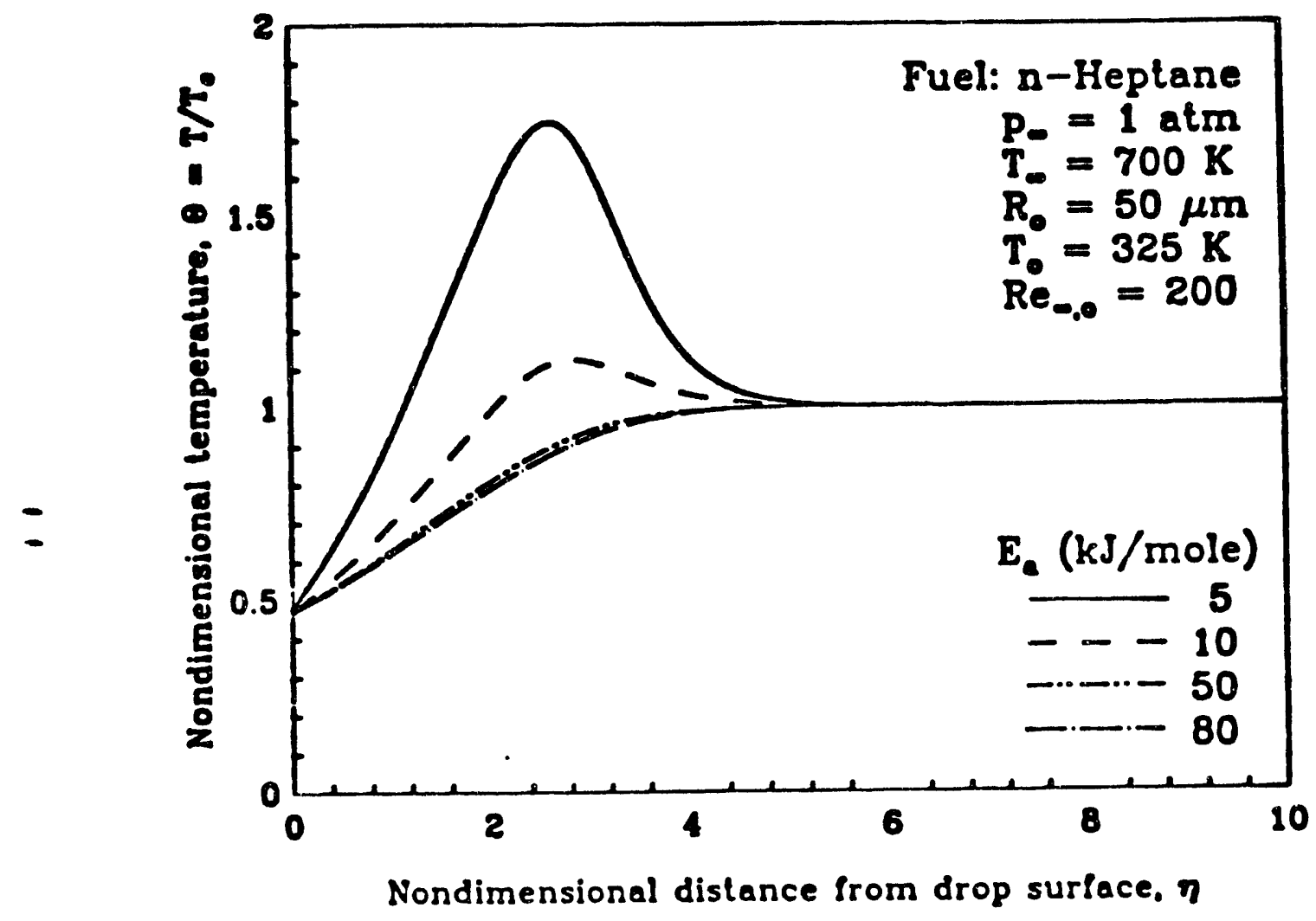

Figure 4-2. Effect of the activation energy on predicted temperature profiles within the boundary layer. 


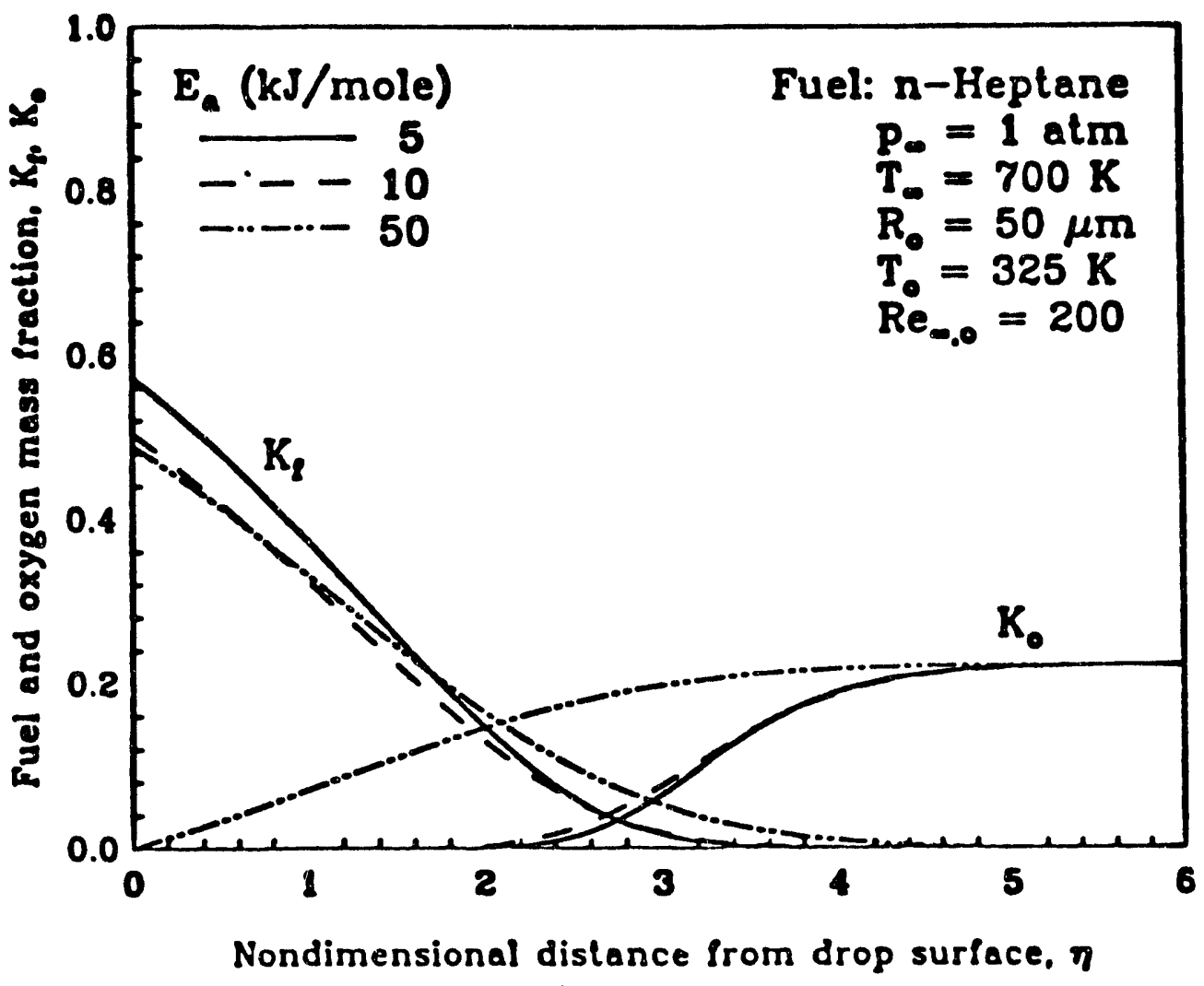

Figure 4-3. Effect of the activation energy on predicted fuel vapor and oxygen mass fraction profiles within the boundary layer. 


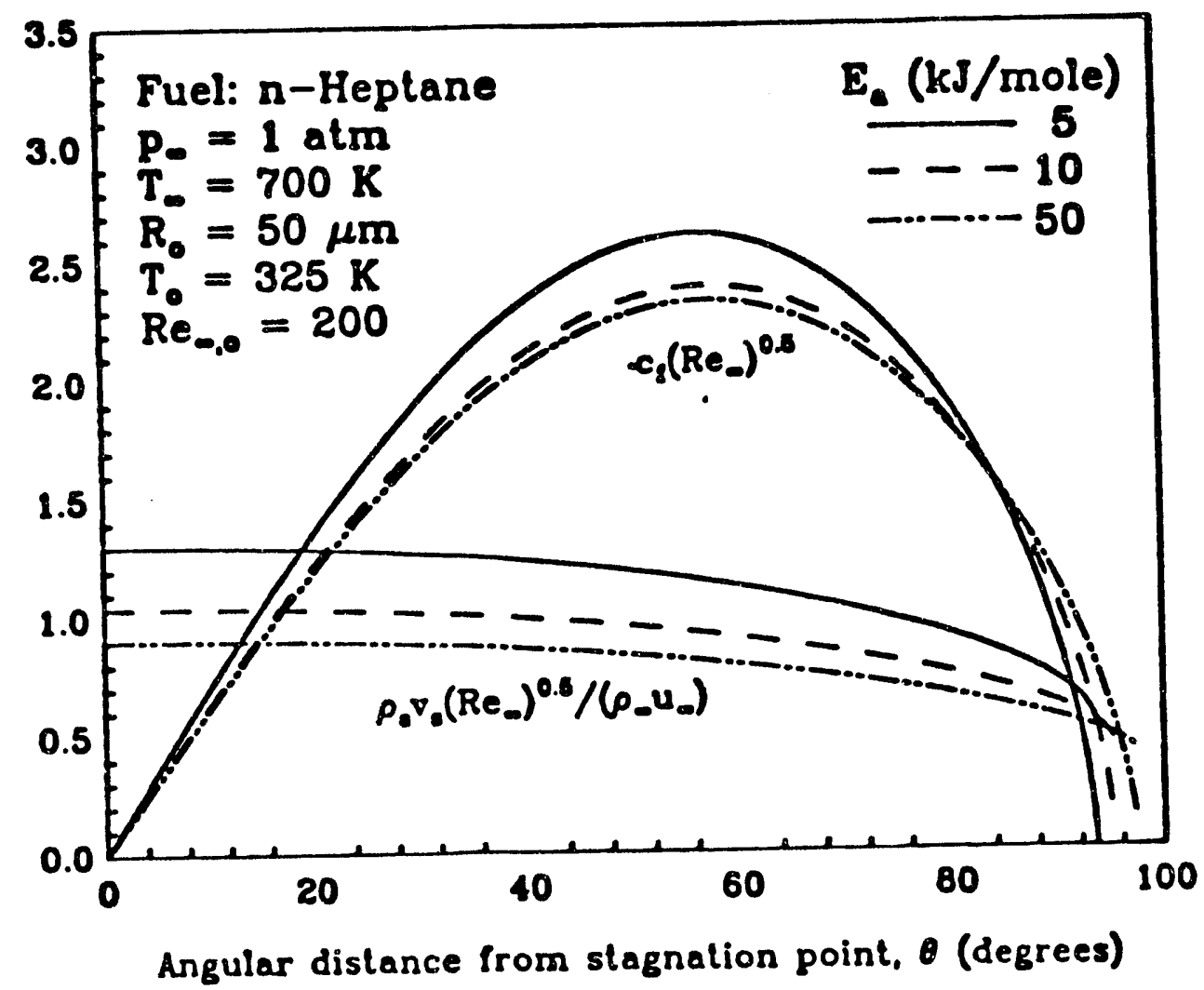

Figure 4-4. Variation of the local skin friction coefficient and mass blowing rate along the droplet surface for different activation energies. 


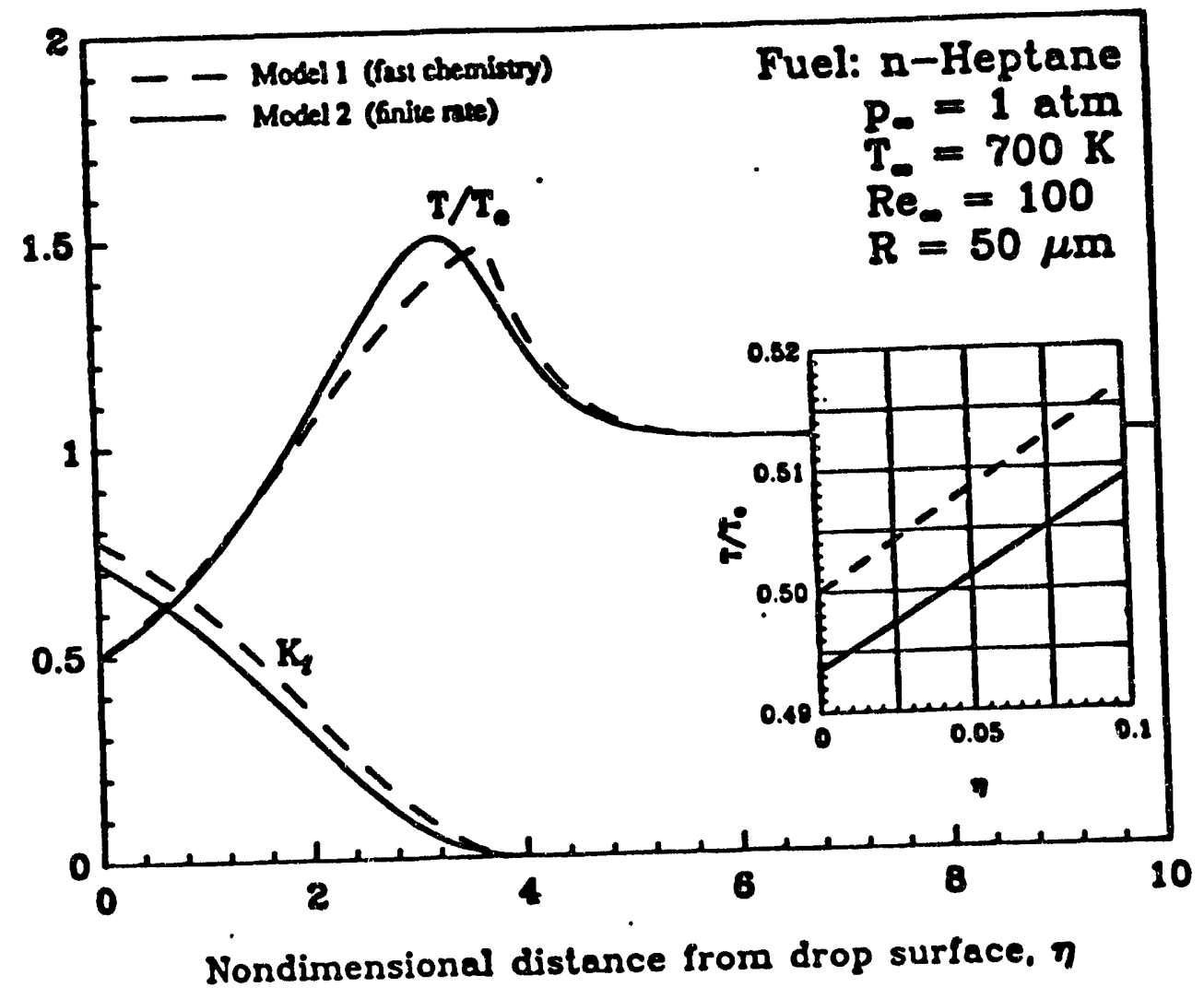

Figure 4-5. Temperature and fuel mass fraction profiles, predicied by Models 1 and 2, at an angular distance $\theta=60^{\circ}$ from the upstream stagnation point. 


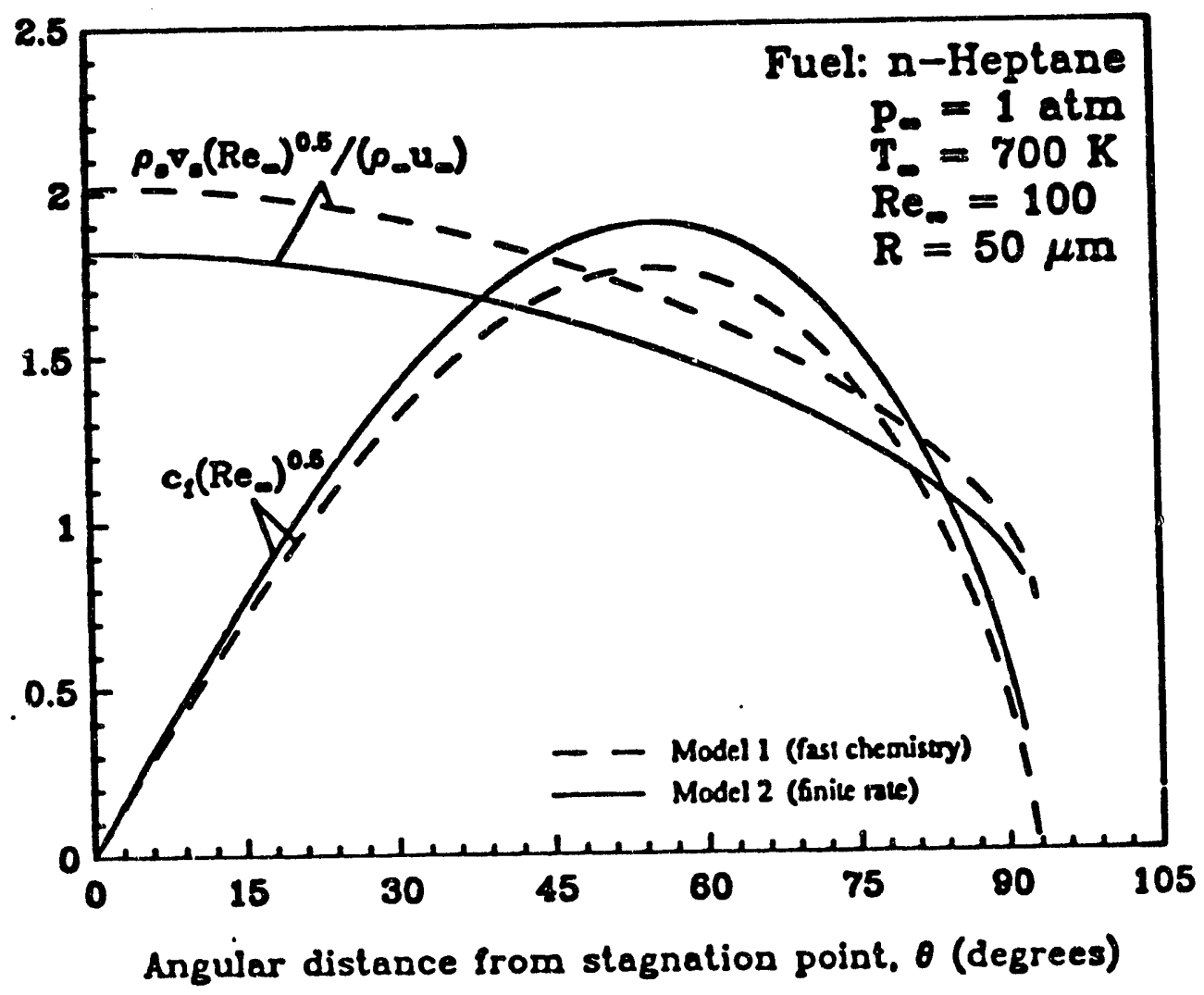

Figure 4-6. Variation of the local mass blowing rate and skin friction coefficient along the droplet surface, as predicted by Models 1 and 2. 


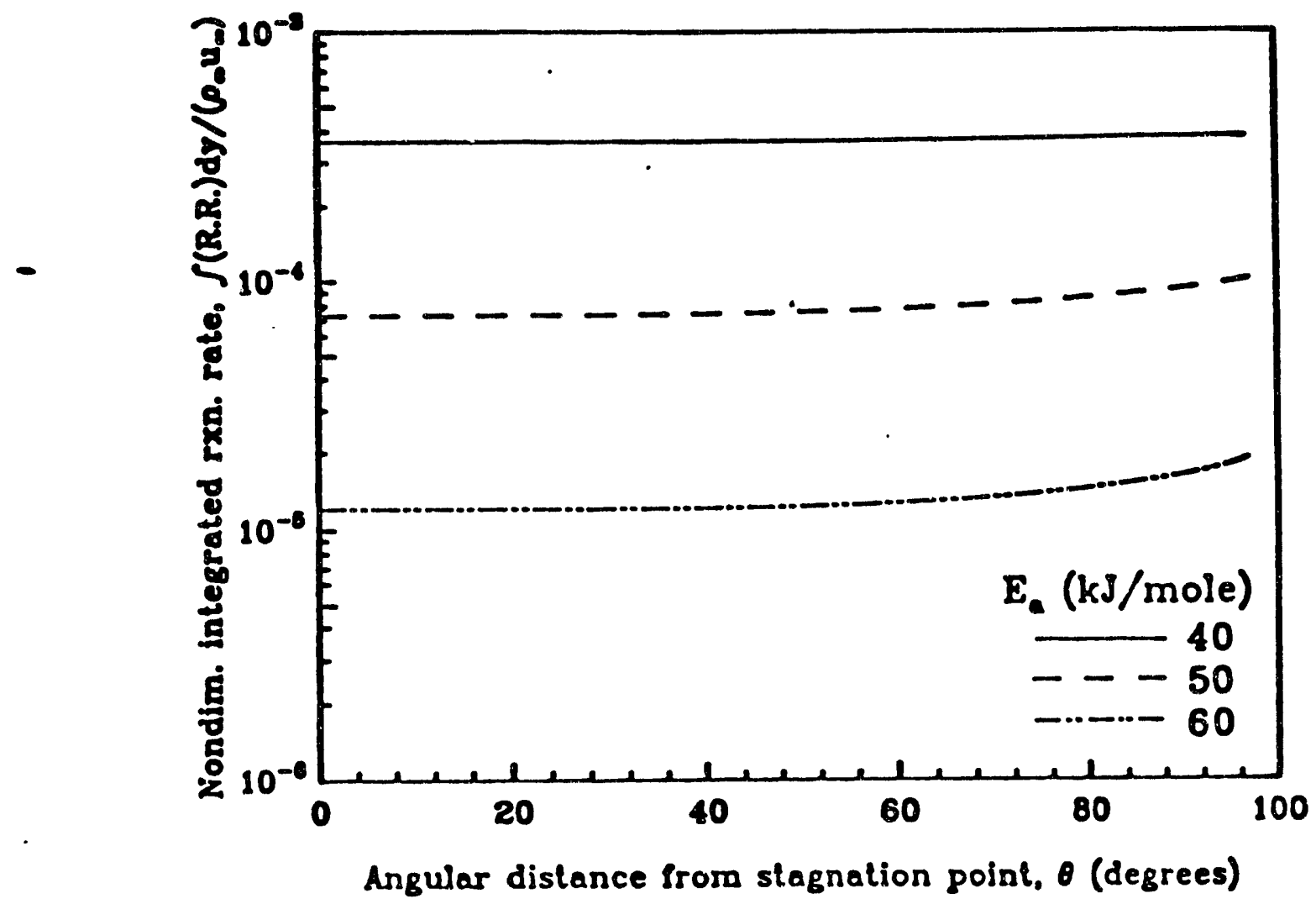

Figure 4-7. Variation of the nondimensional integrated reaction rate along the droplet surface for different activation energies. 


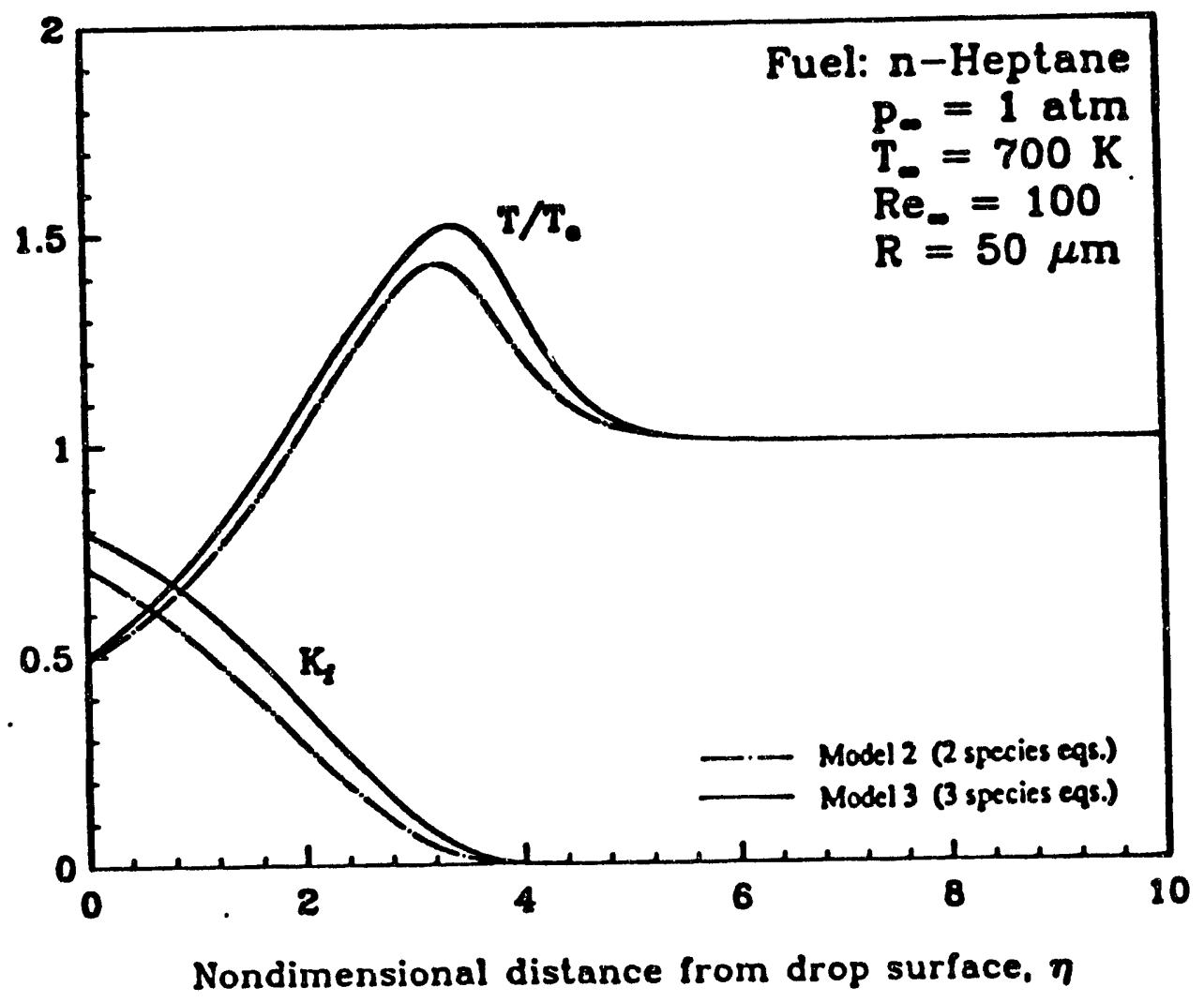

Figure 4-8. Temperature and fuel mass fraction profiles, predicied by Models 2 and 3, at an angular distance $\theta=60^{\circ}$ from the upstream stagnation point. 


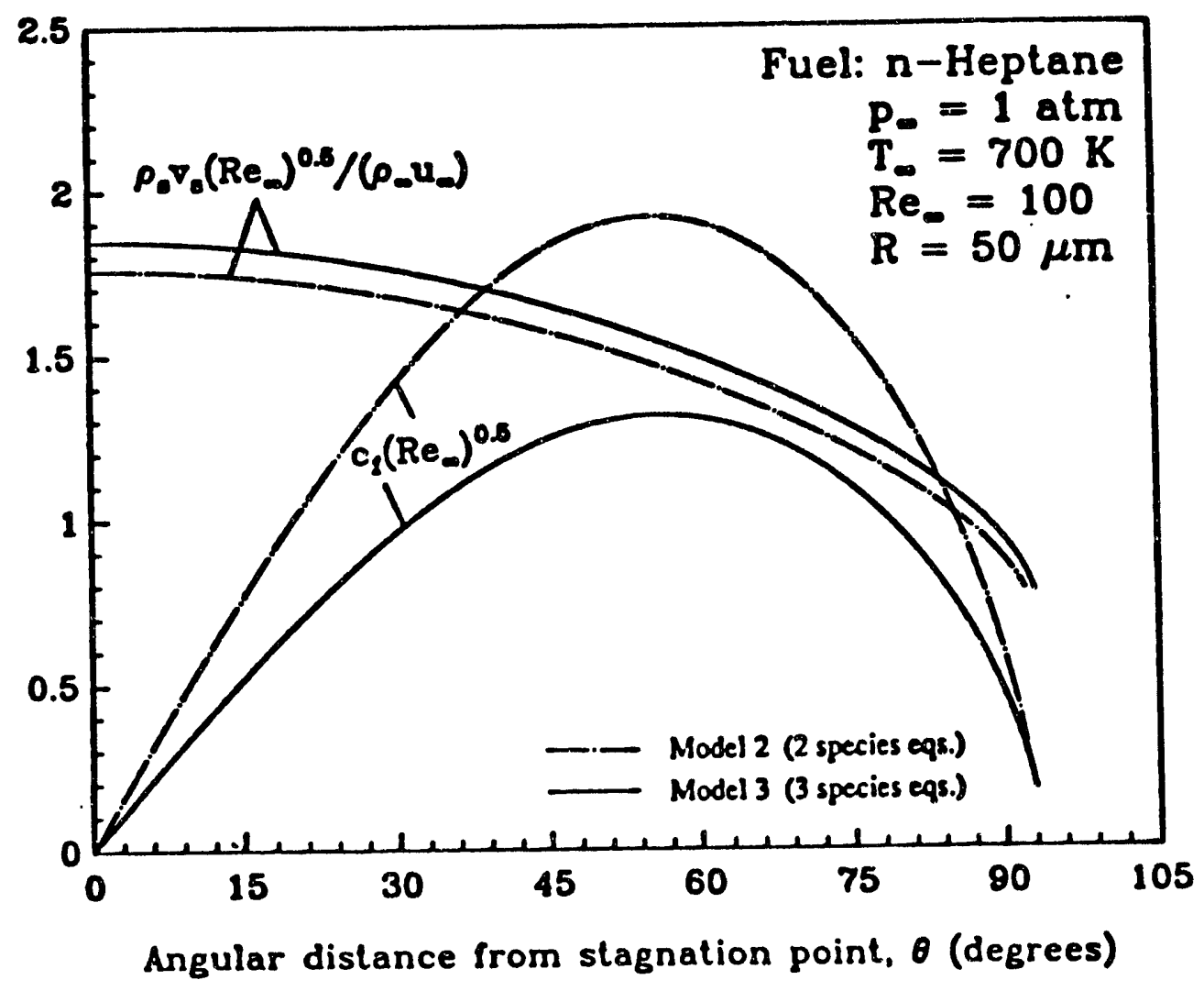

Figure 4-9. Variation of the local and mass blowing rate and skin friction coefficient along the droplet surface, as predicted by Models 2 and 3. 


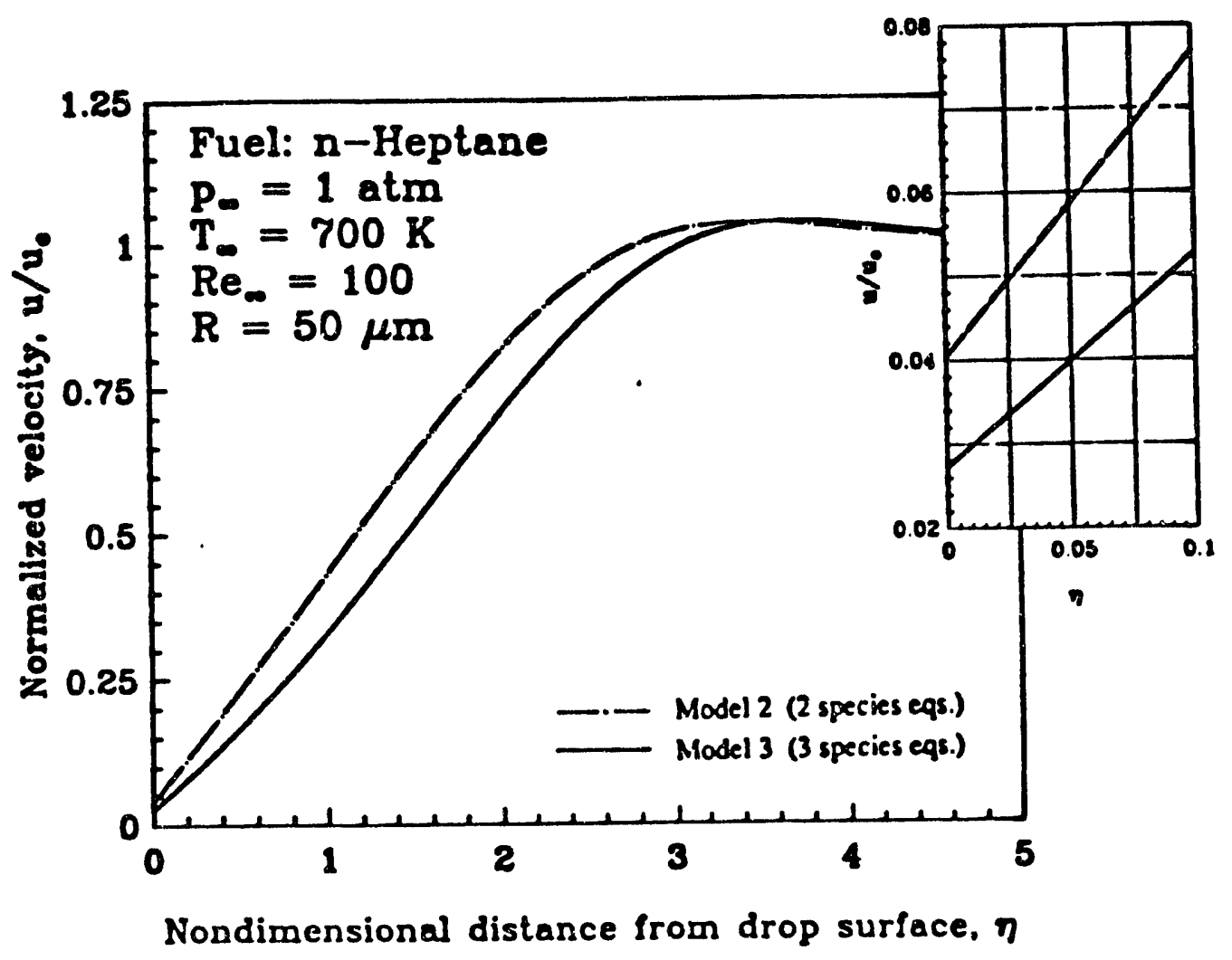

Figure 4-10. Velocity profiles predicted by Models 2 and 3, at an angular distance $\theta=60^{\circ}$ from the upstream stagnation point. 
Force Constants for the Lennard-Jones Potential Modale

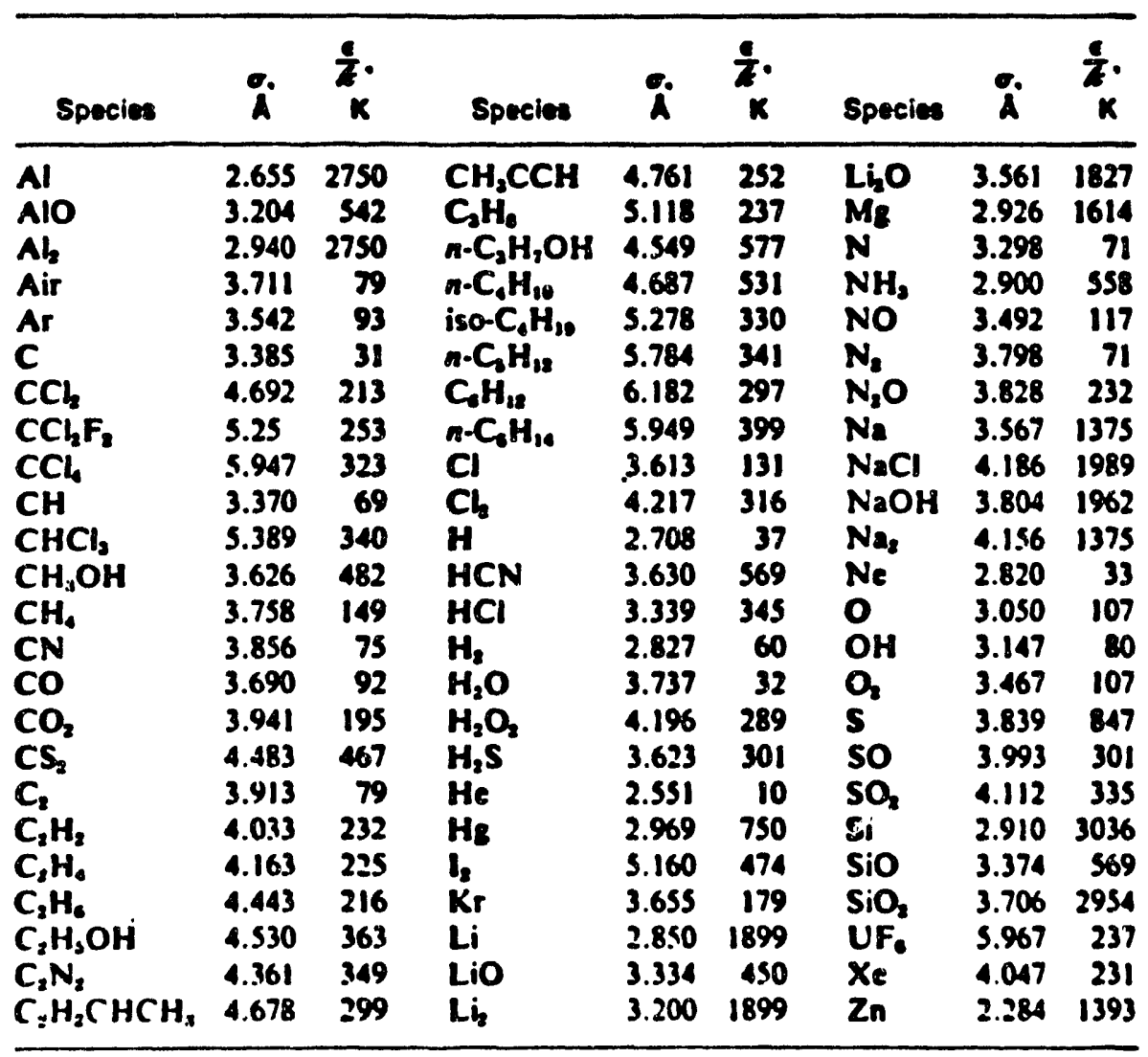

- Tuhen largels from R. A Siehla. NASA TR R.132. 1962.

Table 5-1. Force constants for the Lennard-Jones potential model (from Edwards et al. (1979)). 
Collision Integrals for the Lennard-Jones Potential Modolo

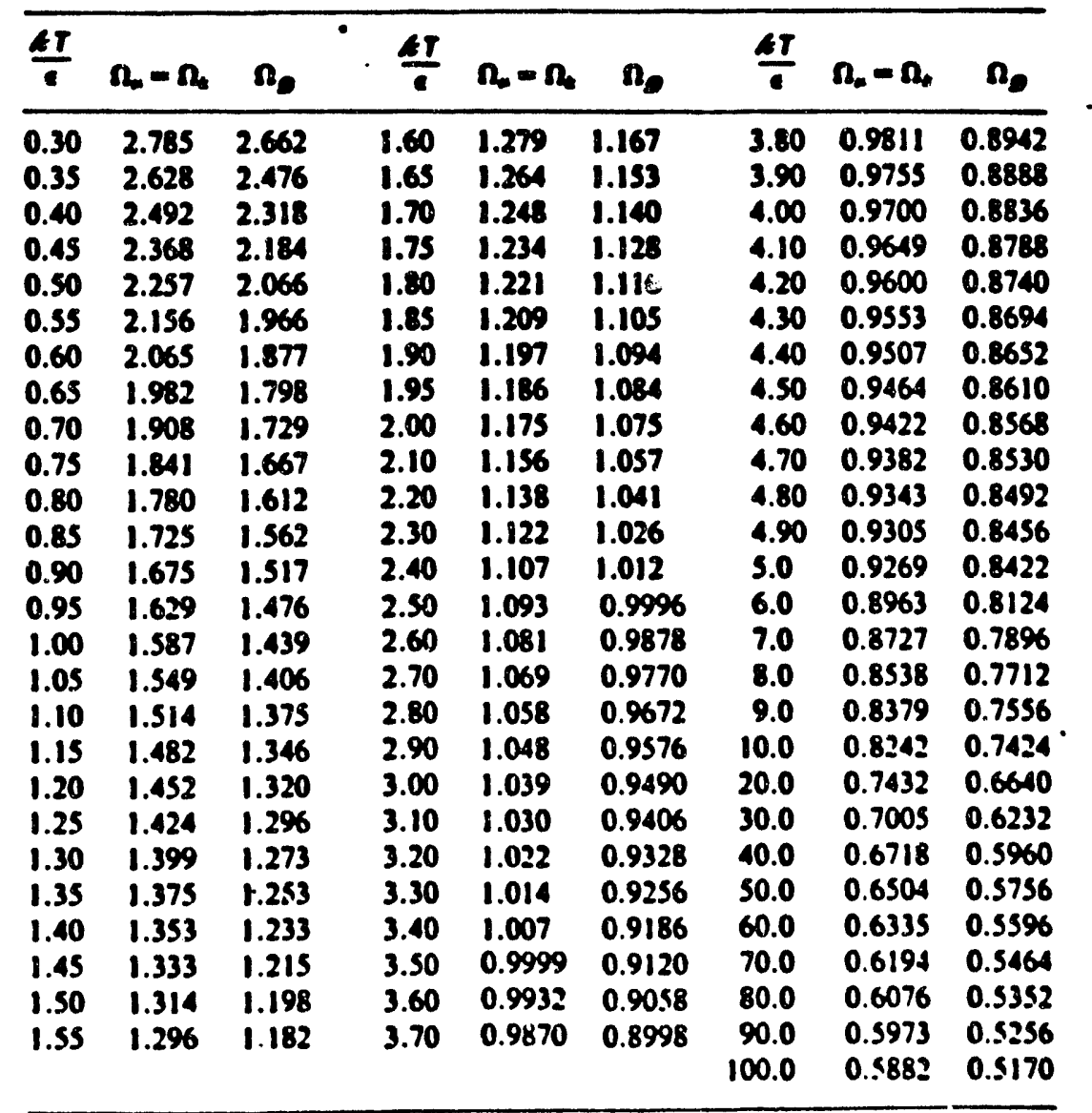

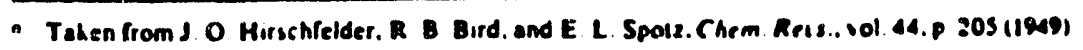

Table 5-2. Collision iniegrals for the Lennard-Jones potential model (from Edwards et al. (1979)). 


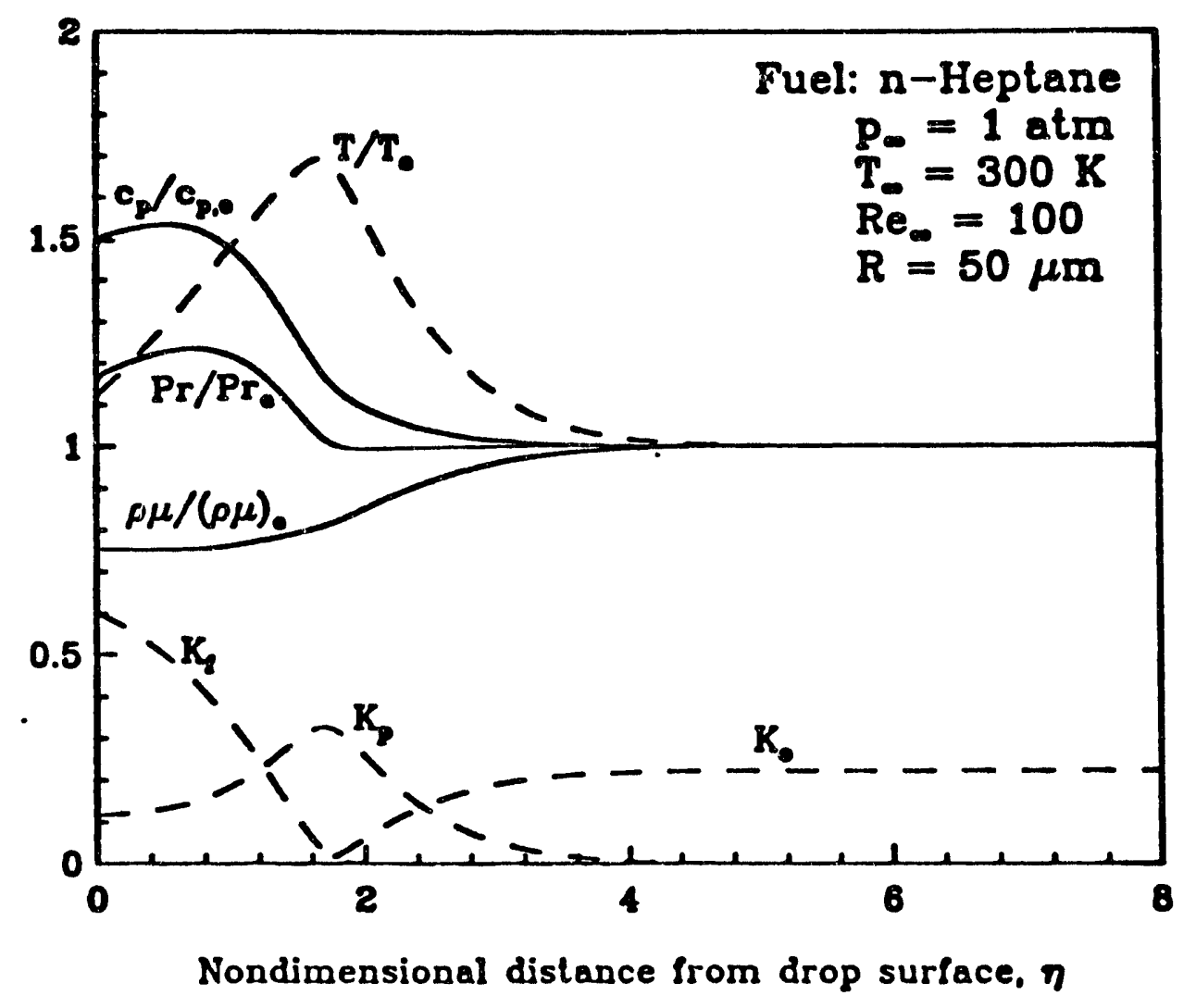

Figure 5-1. Variation of specific heat, Prandll number, $\rho \mu$, temperature, and species mass fractions across the boundary layer at an angular distance $\theta=60^{\circ}$ from the upstream stagnation point. 


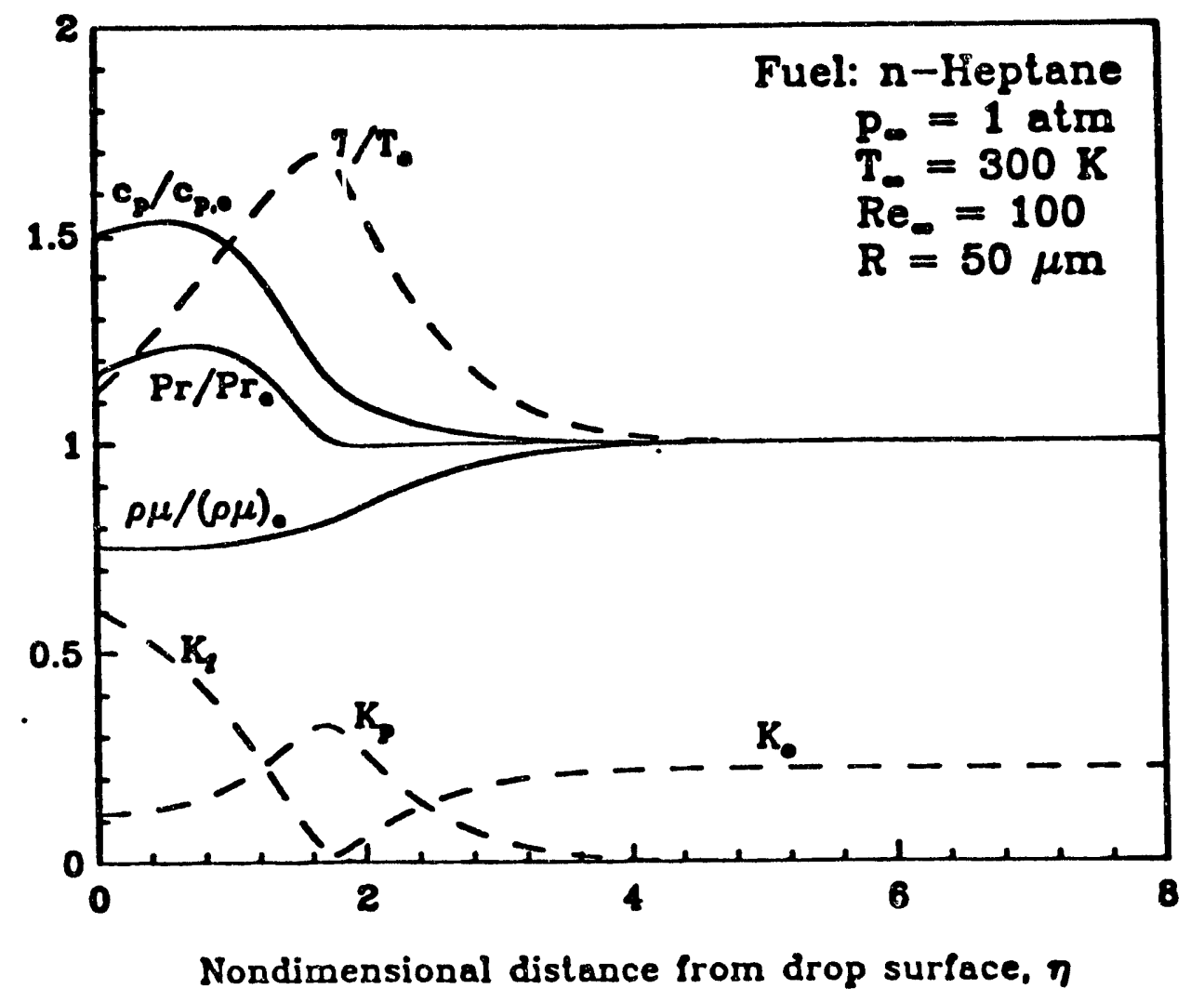

Figure 5-1. Variation of specific heat, Prandil number, $p \mu$, temperature, and species mass fractions across the boundary layer at an angular distance $\theta=60^{\circ}$ from the upstream stagnation point. 


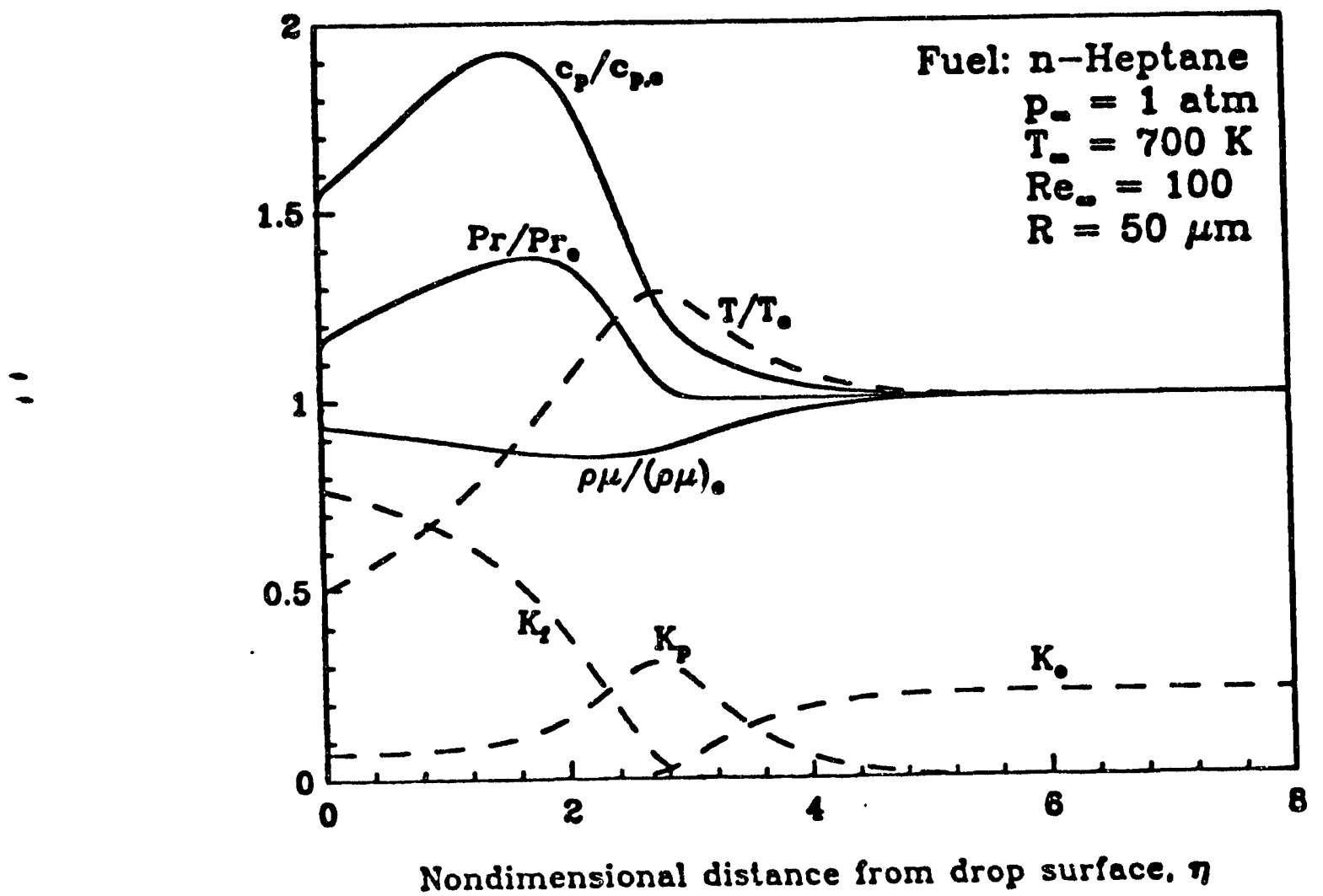

Figure 5-2. Variation of specific heat, Prandll number, $p \mu$, temperature, and species mass fractions across the boundary layer at an angular distance $\theta=60^{\circ}$ from the upstream. 


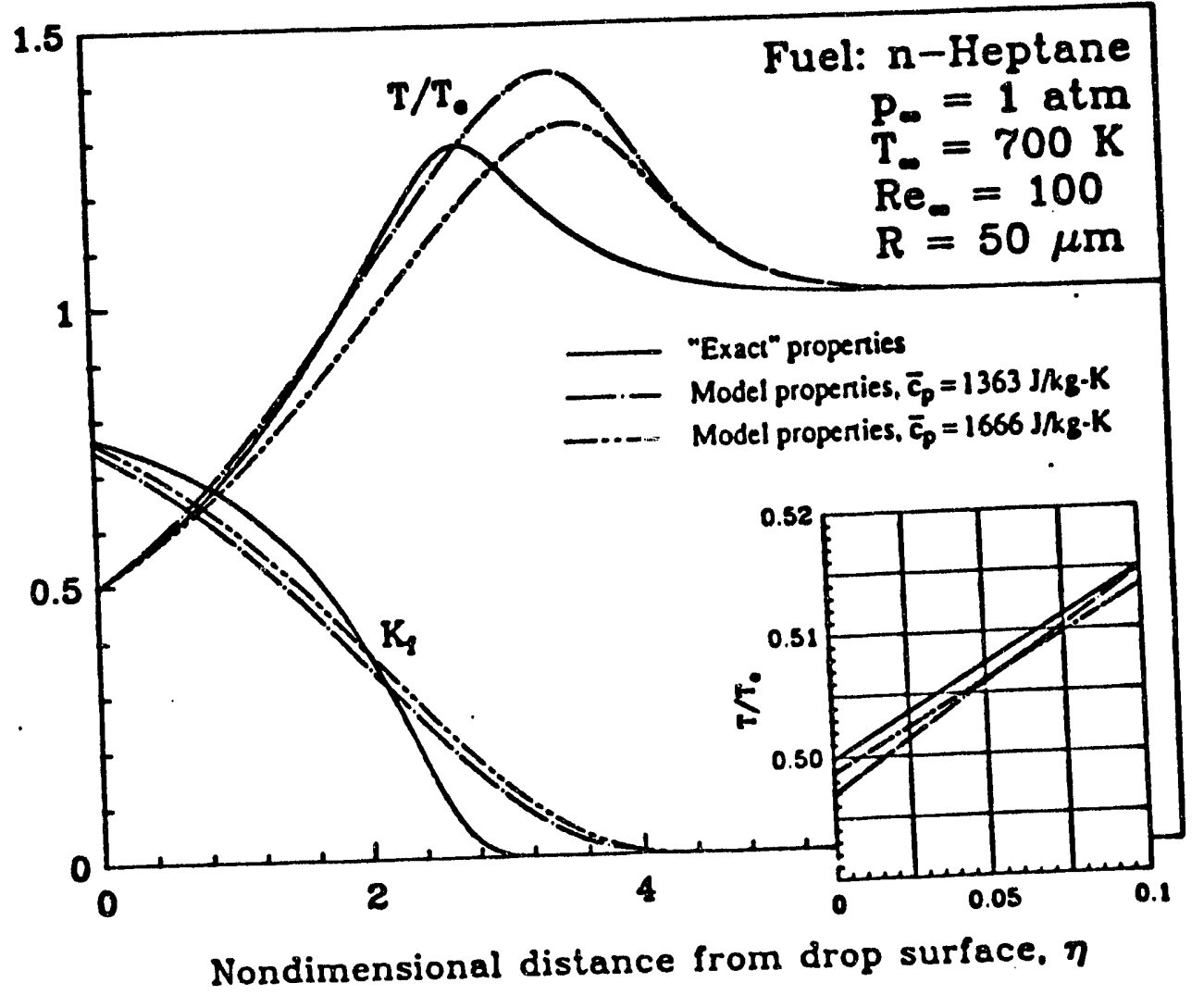

Figure 5-3. Temperature and fuel mass fraction profiles at an angular distance $\theta=60^{\circ}$ from the upstream stagnation point, predicted by Model 4 . and by Model 3 for two different average specific heats. 


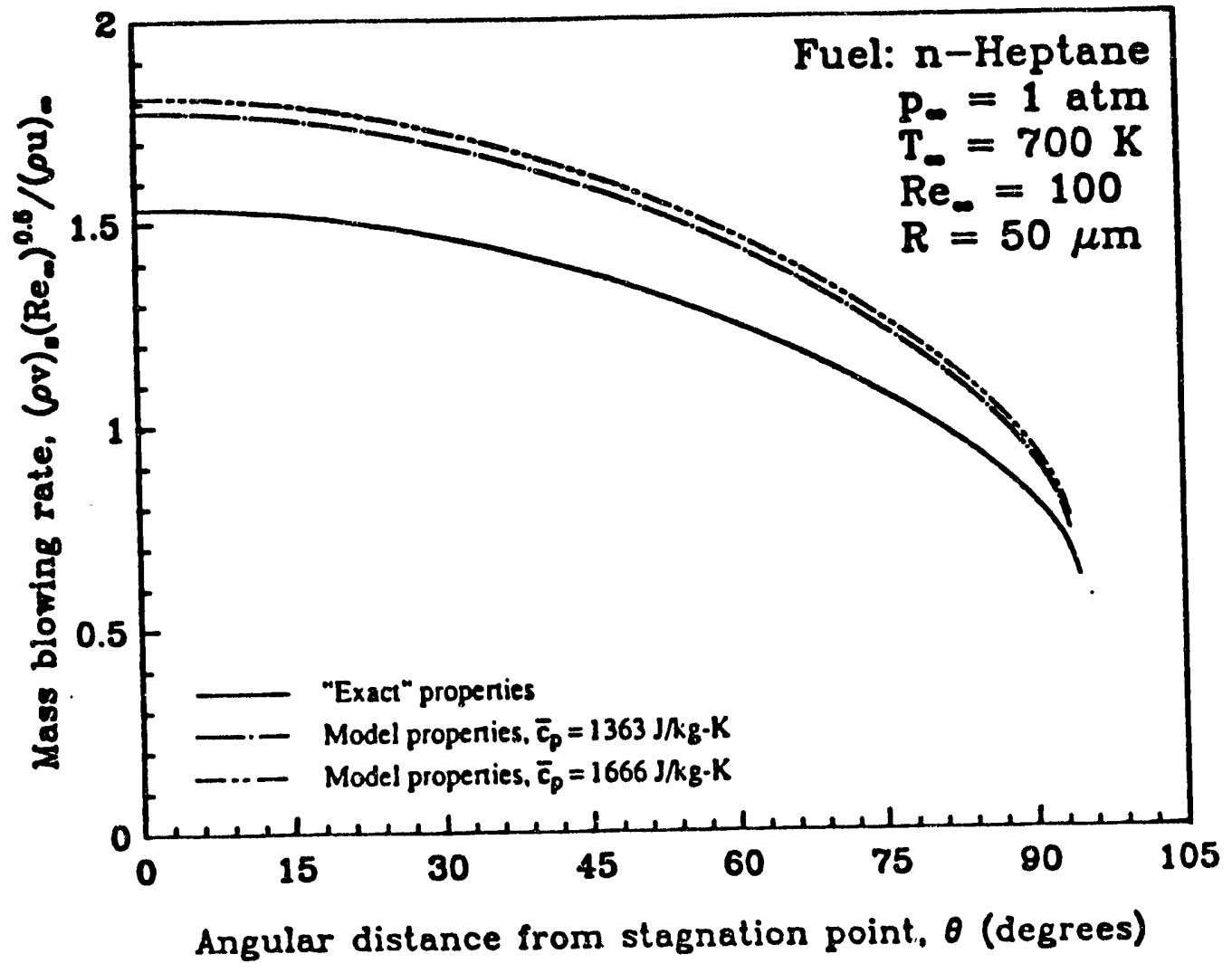

Figure 5-4. Variation of the local mass blowing rate along the droplet surface, as predicted by Model 4, and by Model 3 for two different average specific heats. 


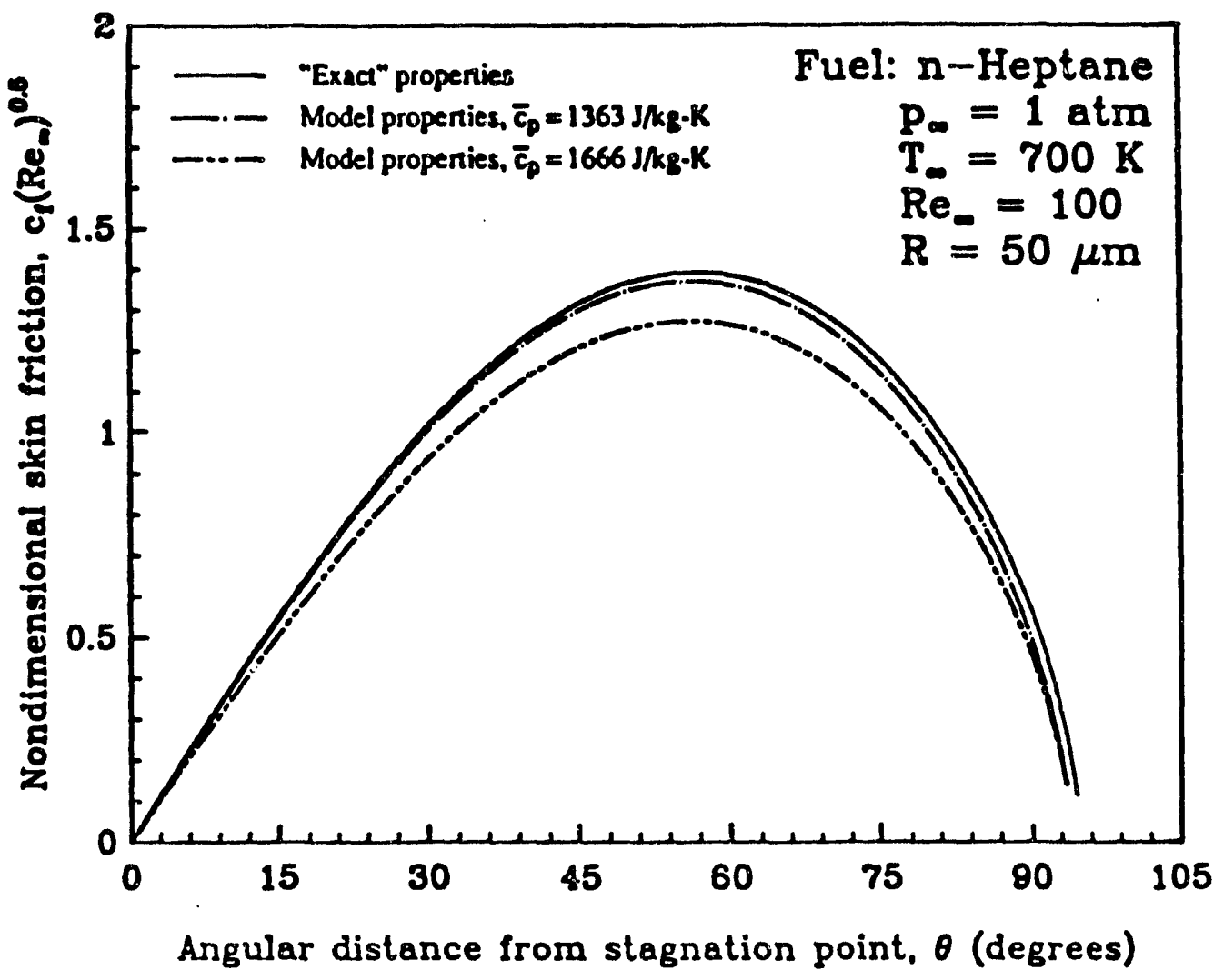

Figure 5-5. Variation of the local skin friction coefficient along the droplet surface, as predicted by Model 4, and by Model 3 for two different average specific heats. 


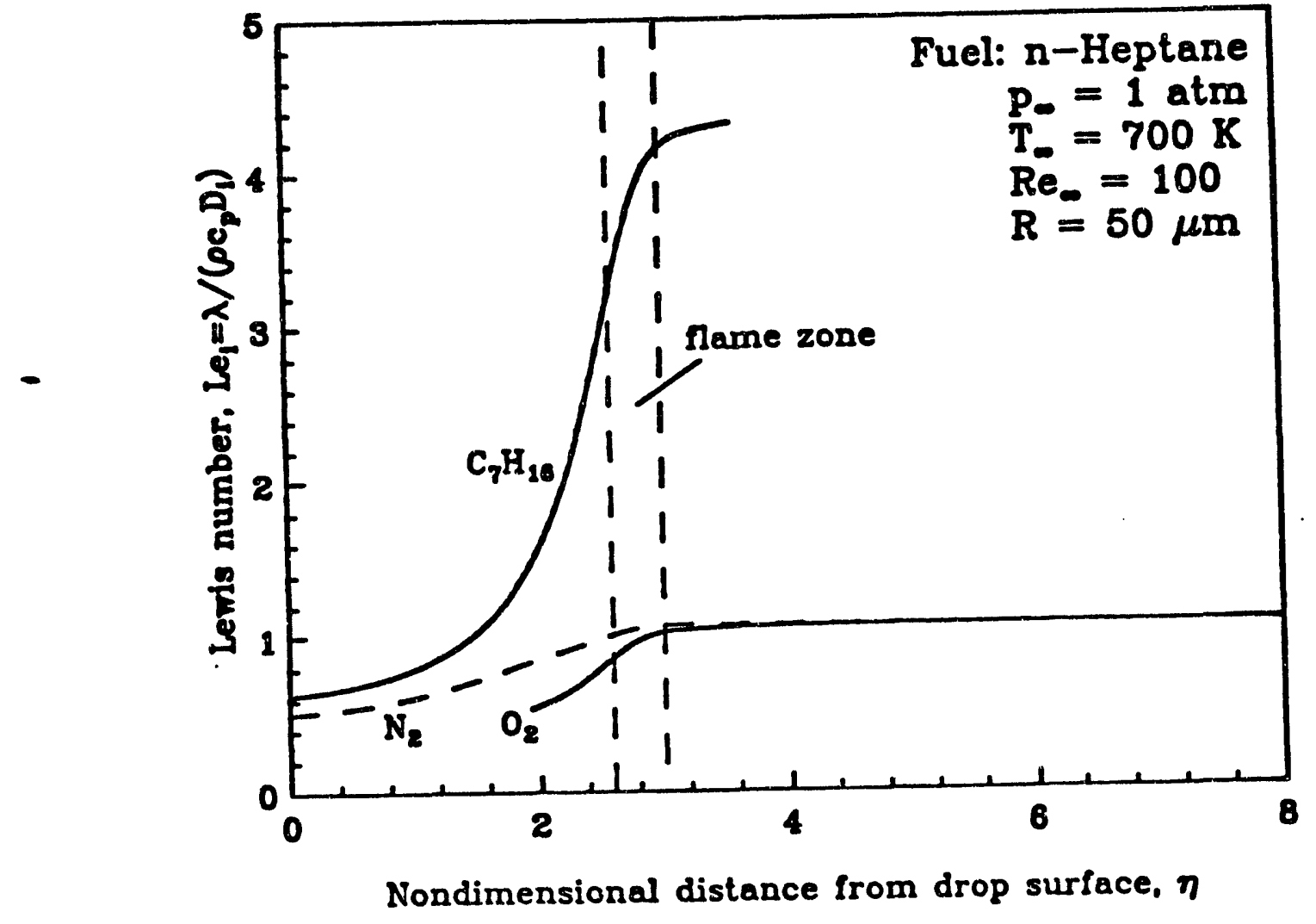

Figure 5-6. Variation of fuel, oxygen and nitrogen Lewis numbers across the boundary layer at an angular distance $\theta=60^{\circ}$ from the upstream stagnation point. 


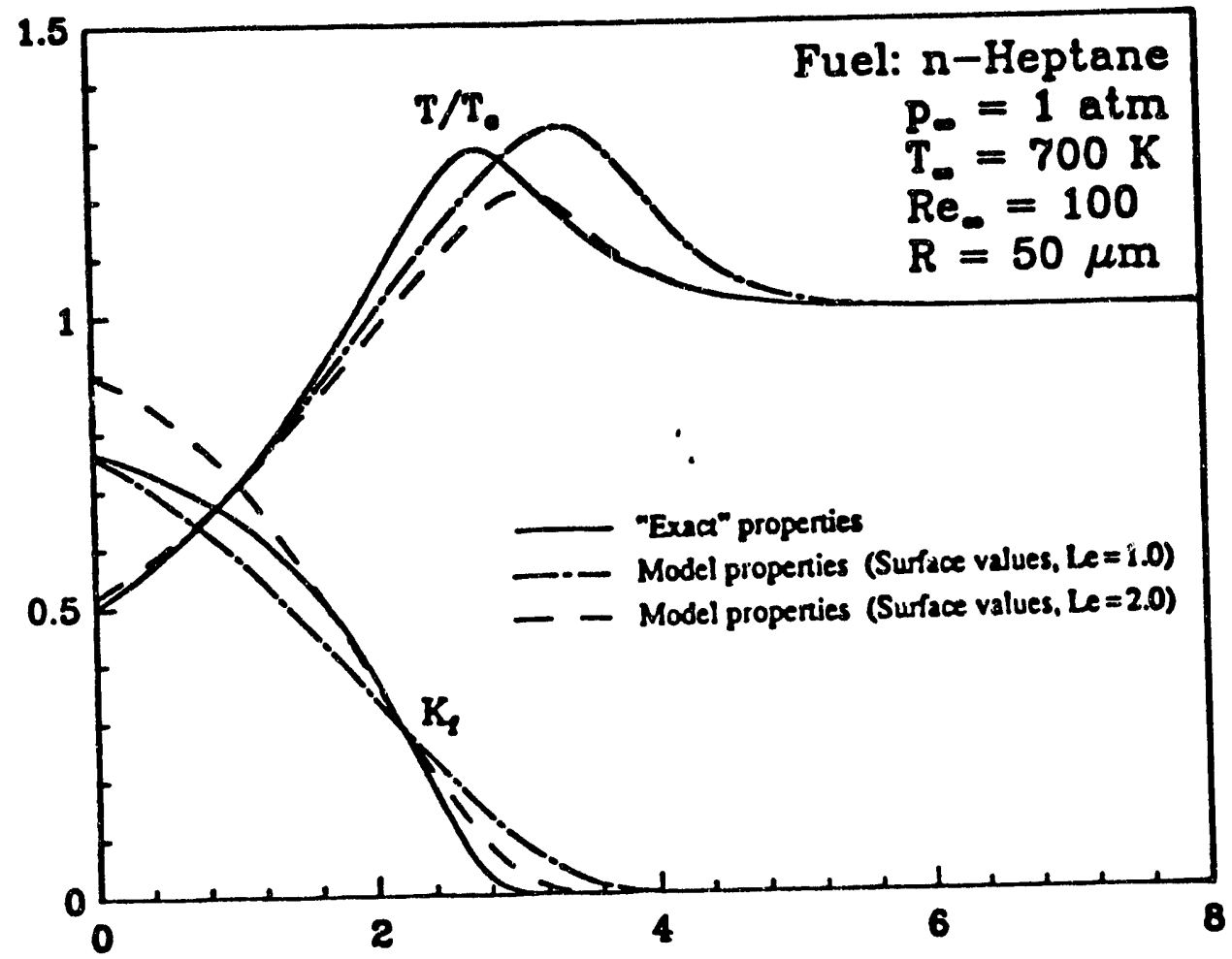

Nondimensional distance from drop surface, $\eta$

Figure 5-7. Temperature and fuel mass fraction profiles at an angular distance $\theta=60^{\circ}$ from the upstream stagnation point, predicted by Model 4 , and by Model 3 for two different effective Lewis numbers. 


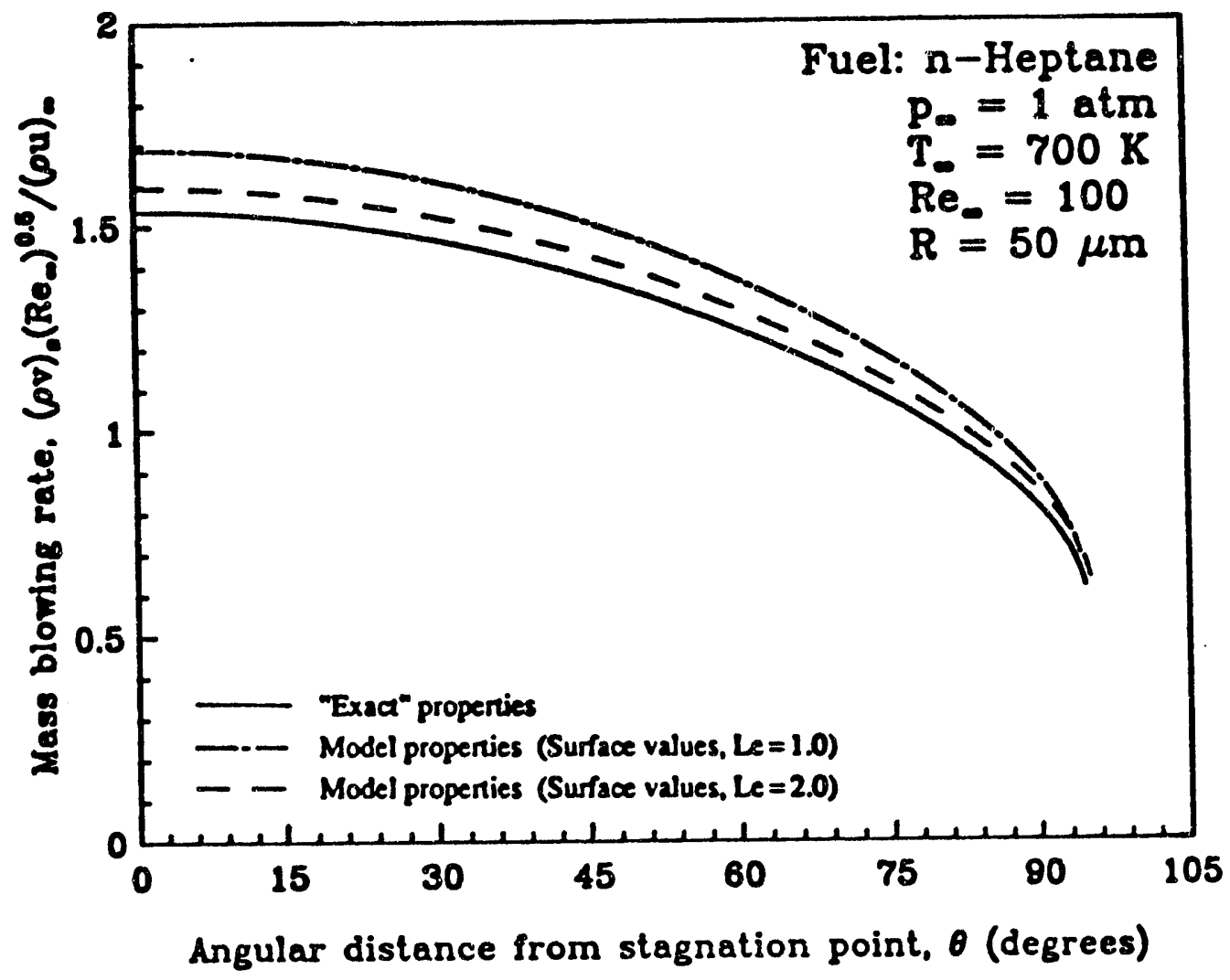

Figure 5-8. Variation of the local mass blowing rate along the droplet surface, as predicted by Model 4, and by Model 3 for two different effective Lewis numbers. 


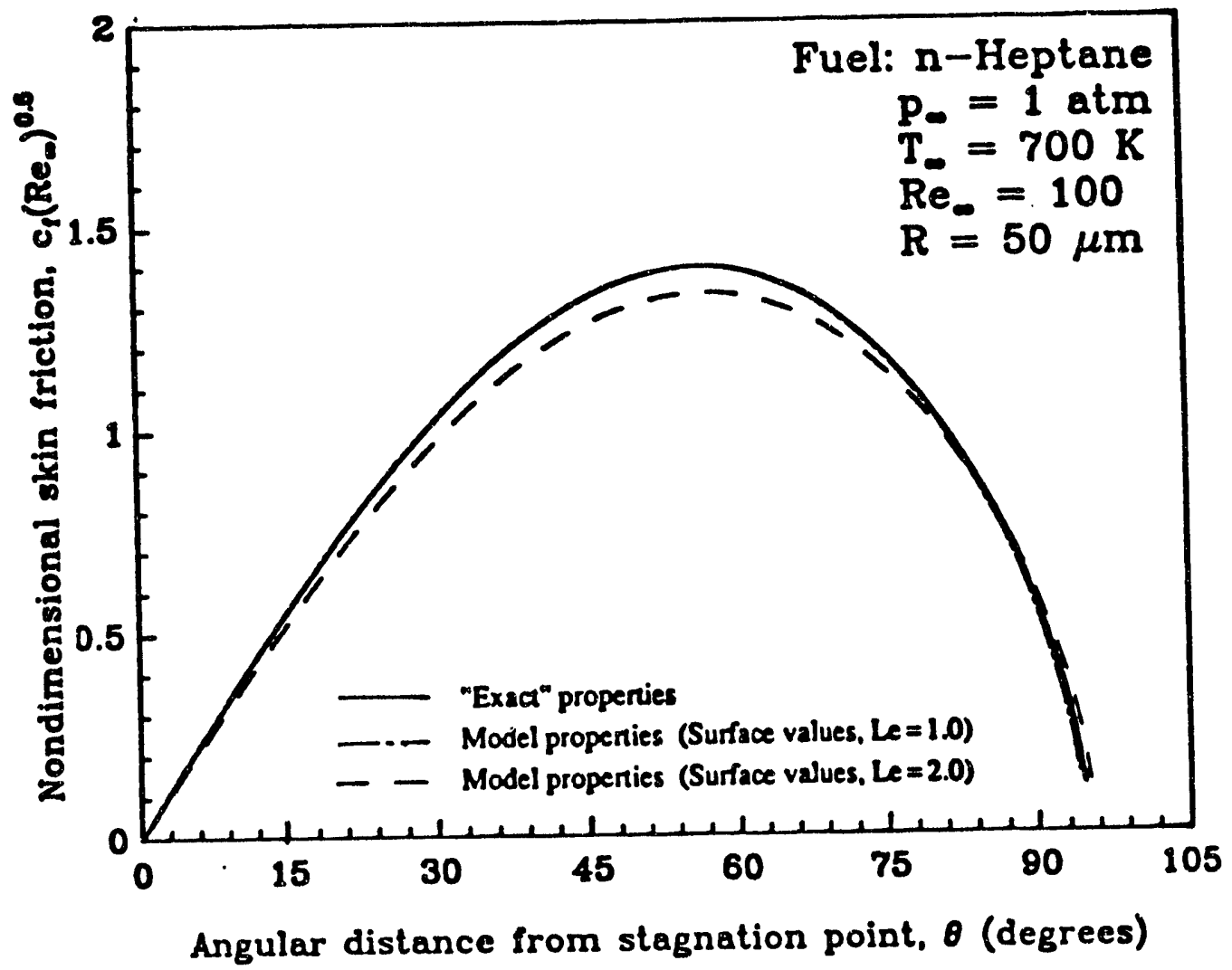

Figure 5-9. Variation of the local skin friction coefficient along the droplet surface, as predicted by Model 4, and by Model 3 for two different effective Lewis numbers. 


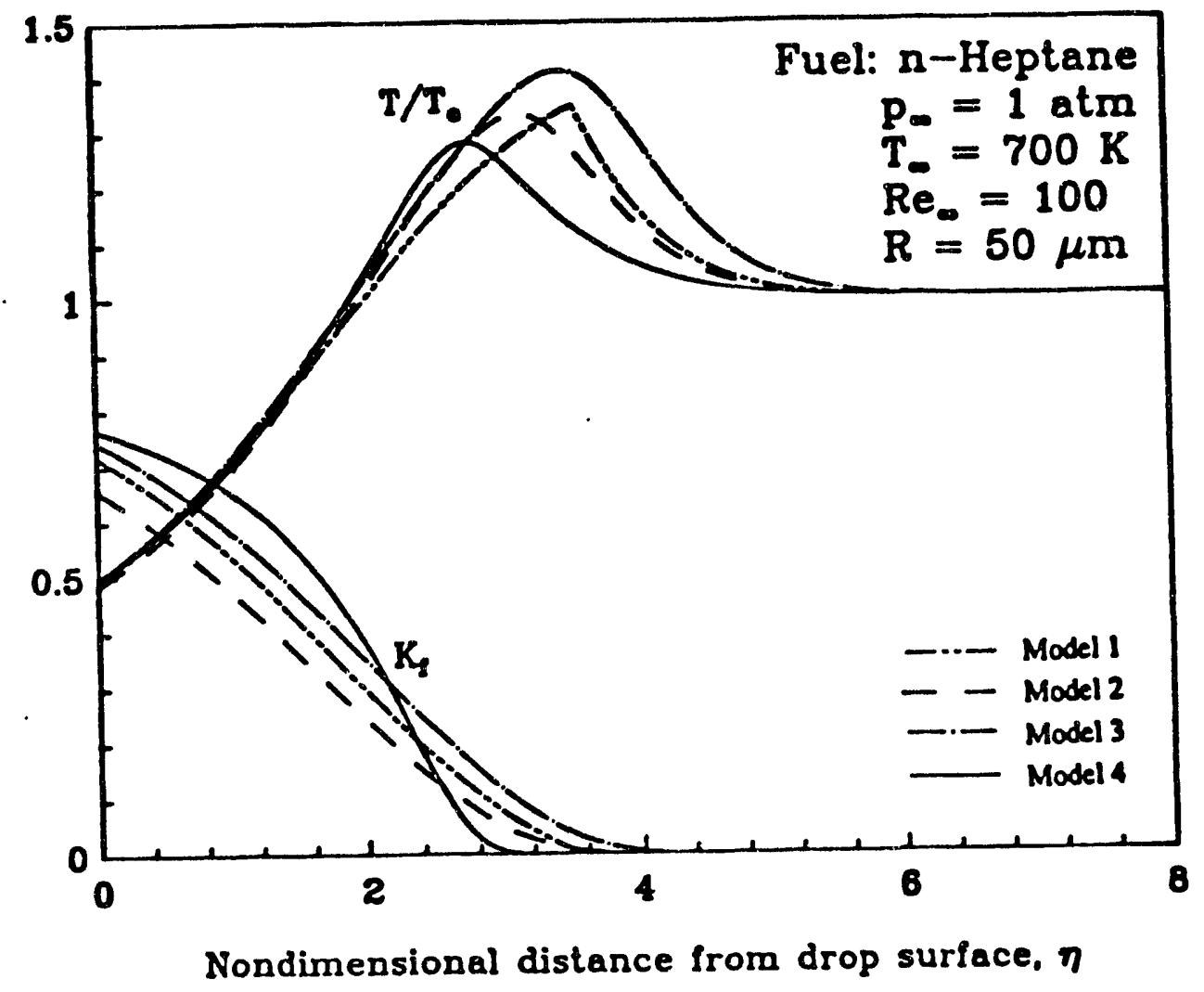

Figure 5-10. Comparison of temperature and fuel mass fraction profiles at an angular distance $\theta=60^{\circ}$ from the upstream stagnation point. predicted by Models 1 to 4. 


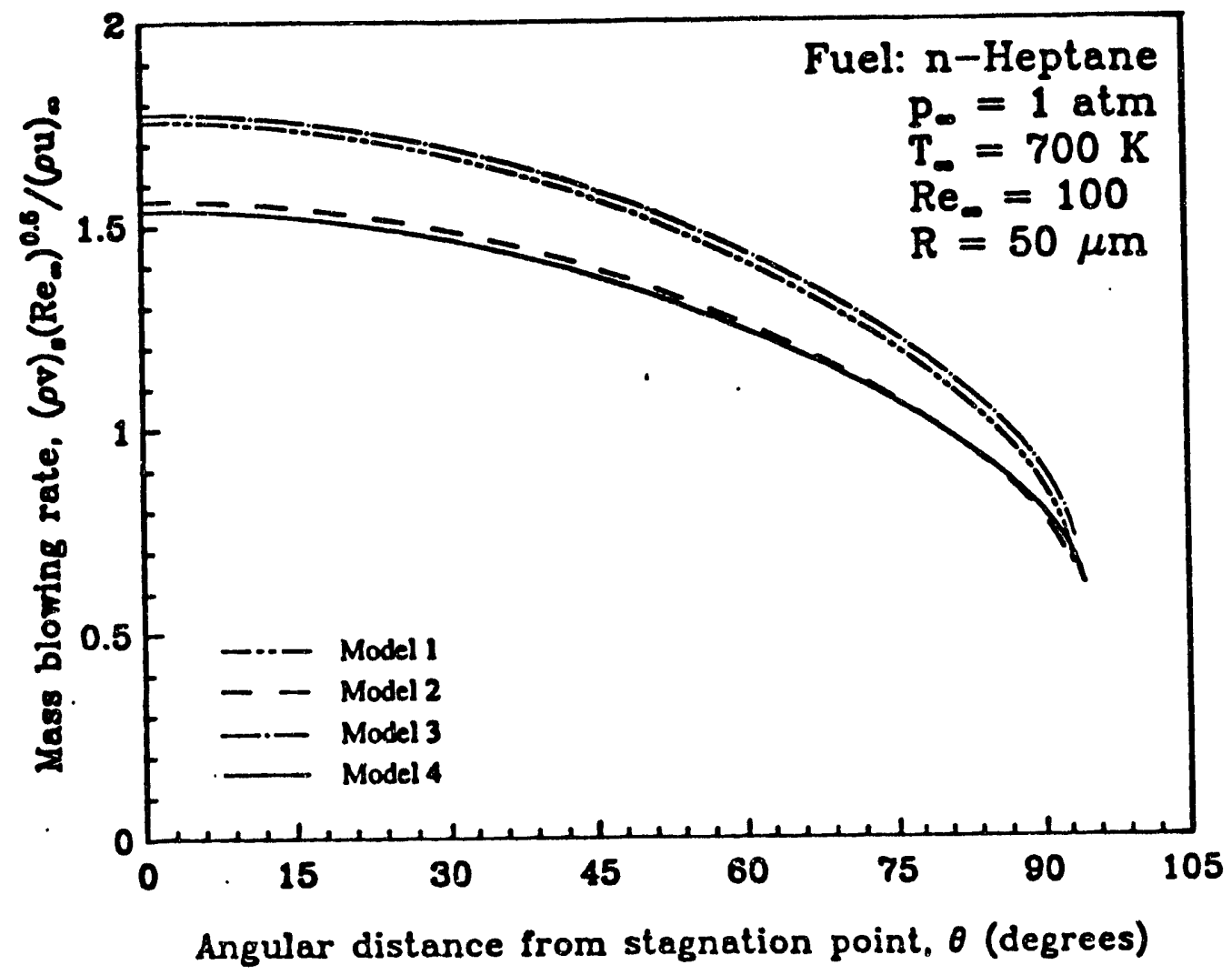

Figure 5-11. Variation of the local mass blowing rate along the droplet surface, as predicted by Models 1 to 4 . 


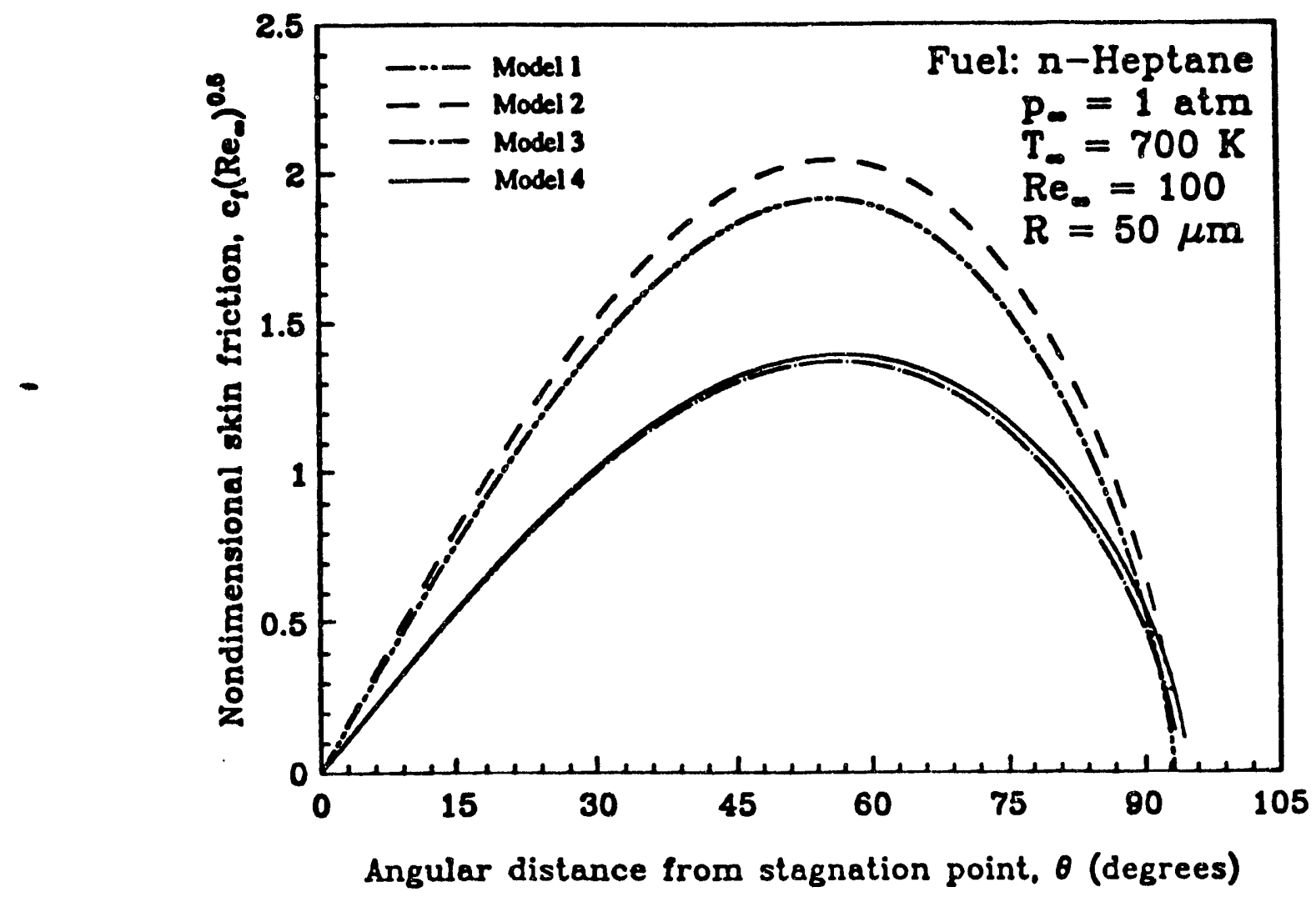

Figure 5-12. Variation of the local skin friction coefficient along the droplet surface, as predicted by Models 1 to 4. 


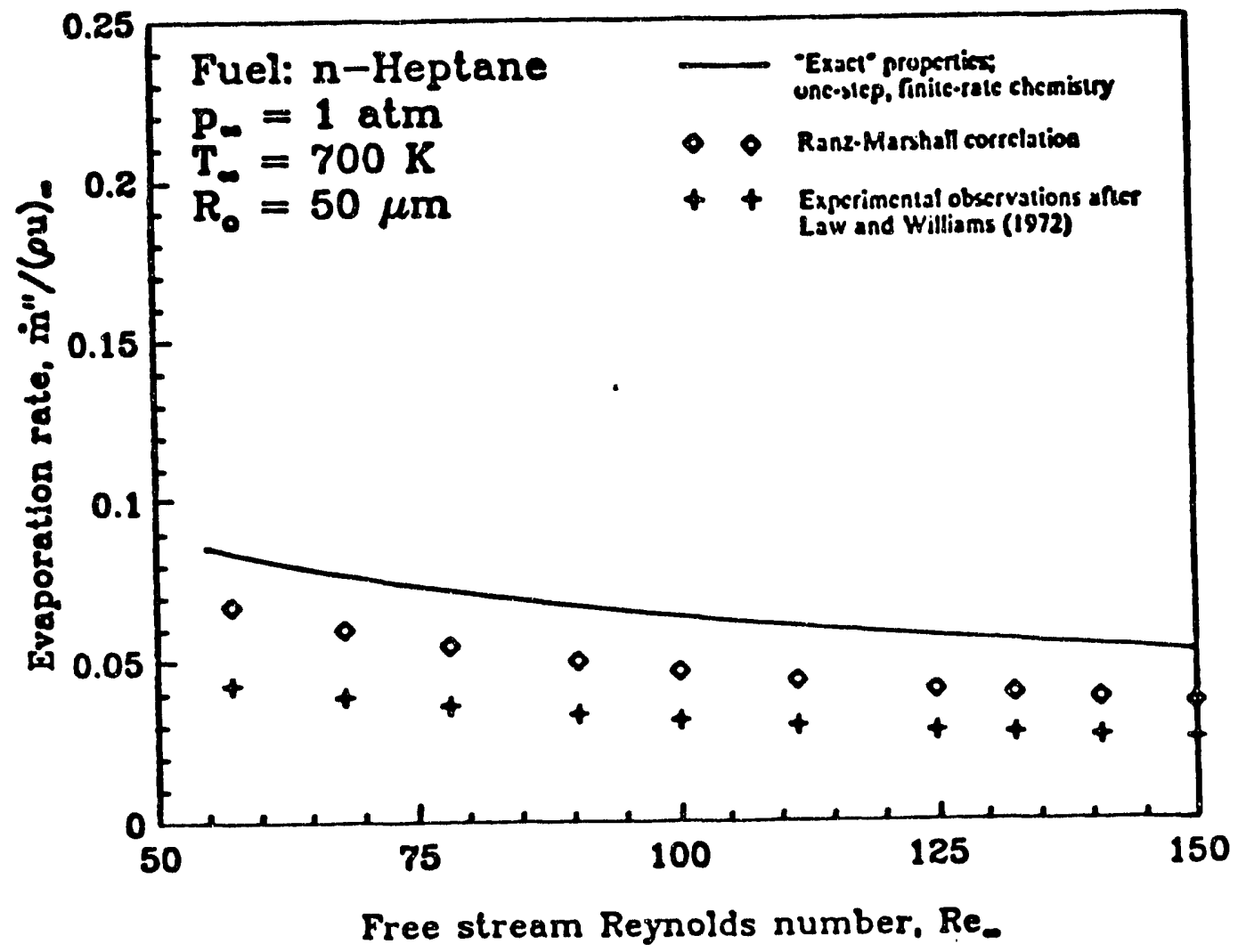

Figure 5-13. Predicted variation in mass transfer rate from the buming droplet with Reynolds number at $700 \mathrm{~K}$. Comparison is made with the experimental observations of Law and Williams (1972), and the Froessling/Ranz-Marshall correlation. 


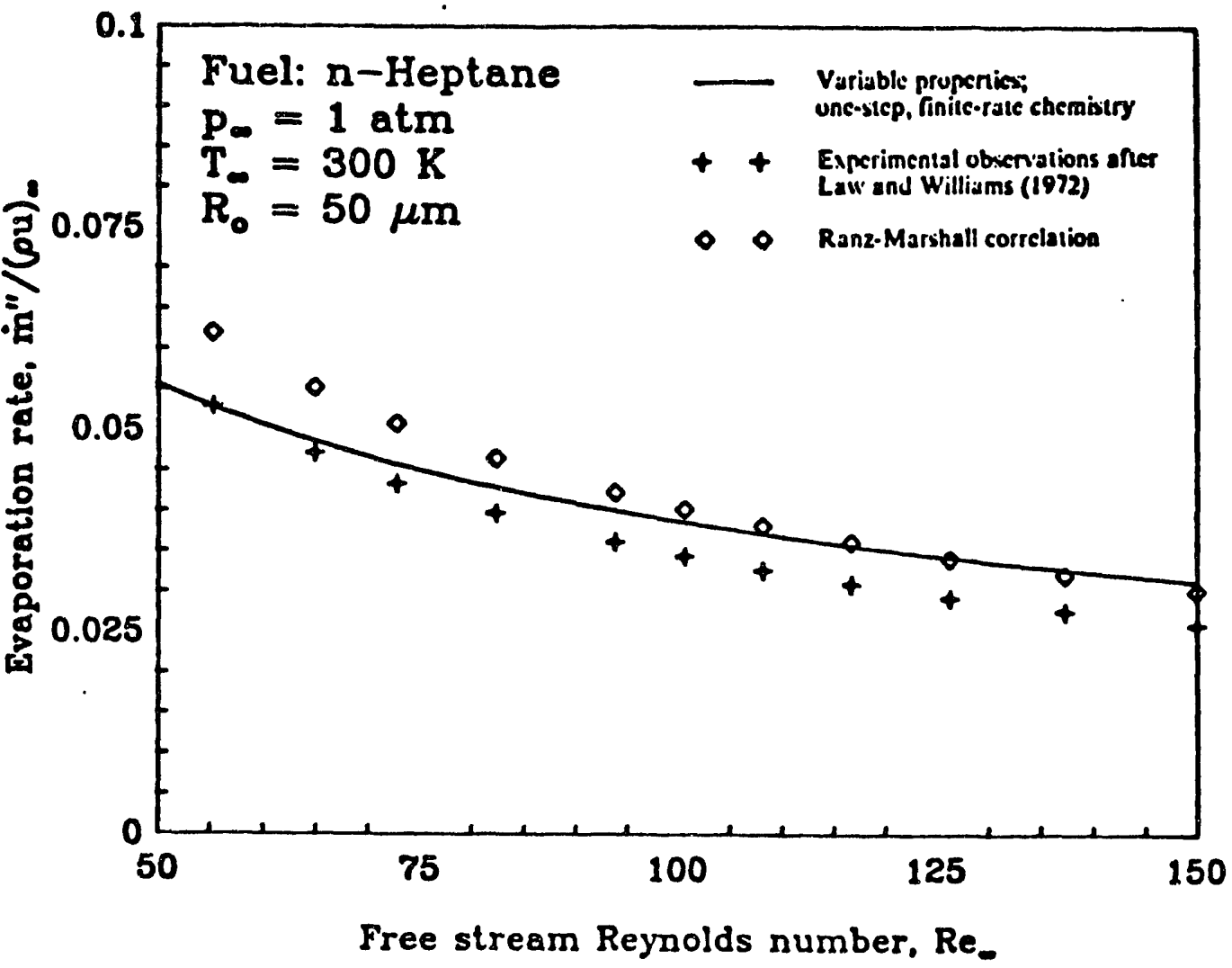

Figure 5-14. Predicted variation in mass transfer rate from the buming droplet with Reynolds number at $300 \mathrm{~K}$. Comparison is made with the experimental observations of Law and Williams (1972), and the Froessling/Ranz-Marshall correlation. 


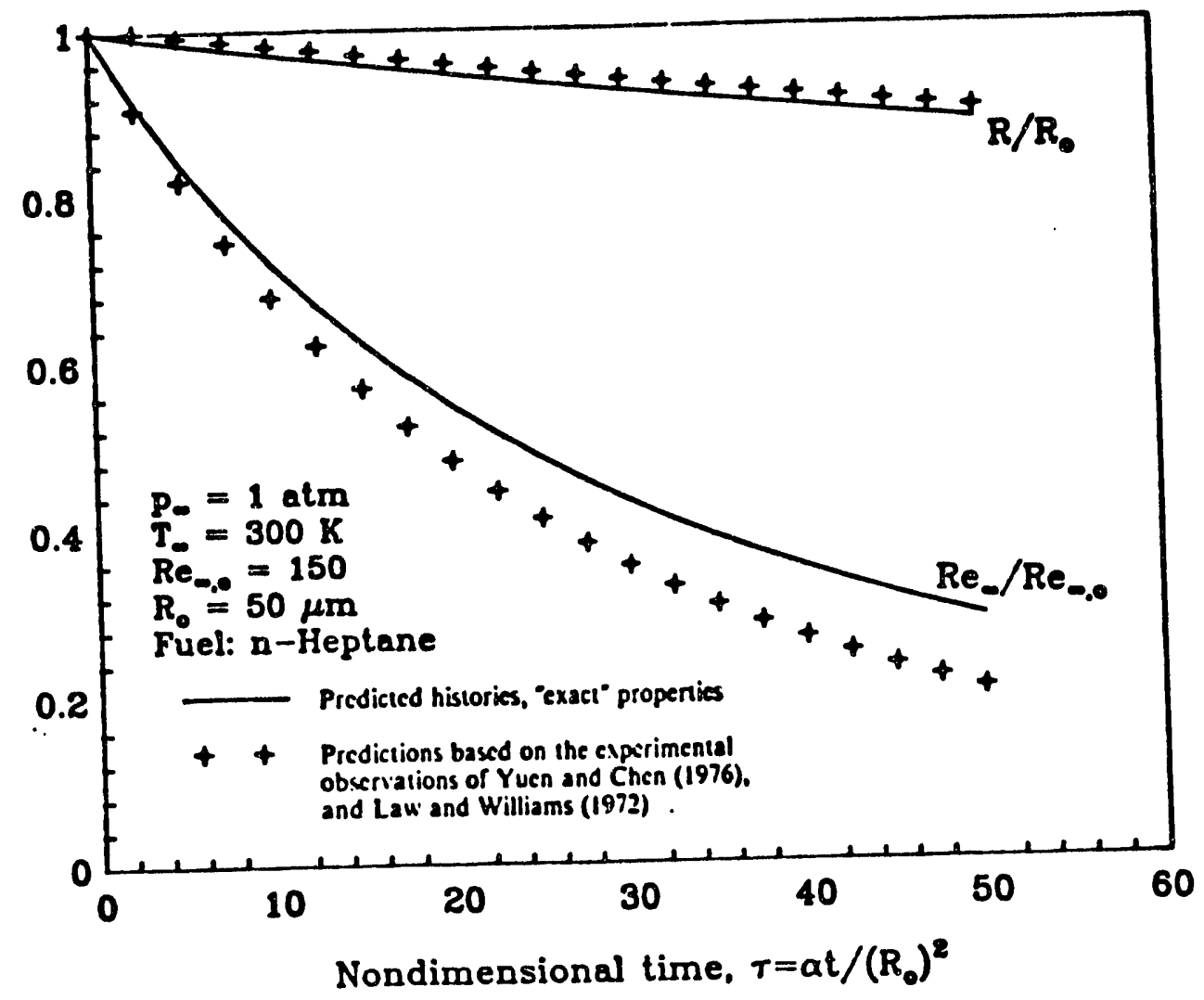

Figure 5-15. Predicted droplet histories (radius and Reynolds number) for a buming droplet at $300 \mathrm{~K}$. Comparison is made with the experimental data of Law and Willians (1972), using the data on drag coefficient from Yuen and Chen (1976). 


\begin{tabular}{|c|c|c|c|c|}
\hline Reference & $\begin{array}{c}\text { Type of } \\
\text { Model }\end{array}$ & $\begin{array}{c}\text { Transient } \\
\text { Heating? }\end{array}$ & $\begin{array}{c}\text { Chemical } \\
\text { Reaction? }\end{array}$ & $\begin{array}{c}\text { "Exact" } \\
\text { Properties? }\end{array}$ \\
\hline $\begin{array}{c}\text { Prakash and } \\
\text { Sirignano (1980) }\end{array}$ & $\begin{array}{c}\text { boundary } \\
\text { layer }\end{array}$ & yes & no & no \\
\hline $\begin{array}{c}\text { Renksizbulut } \\
\text { and Yuen (1983) }\end{array}$ & $\begin{array}{c}\text { fully } \\
\text { numerical }\end{array}$ & no & no & no \\
\hline $\begin{array}{c}\text { Rangel and } \\
\text { Fernandez-Pello (1984) }\end{array}$ & $\begin{array}{c}\text { boundary } \\
\text { layer }\end{array}$ & no & fast & no \\
\hline $\begin{array}{c}\text { Dwyer/Sanders } \\
\text { (1984-90) } \\
\text { fully } \\
\text { numerical }\end{array}$ & yes & $\begin{array}{c}\text { finite } \\
\text { rate }\end{array}$ & no \\
\hline $\begin{array}{c}\text { Haywood et al. } \\
\text { (1989) }\end{array}$ & $\begin{array}{c}\text { fully } \\
\text { numerical }\end{array}$ & yes & no & yes \\
\hline $\begin{array}{c}\text { Huang and } \\
\text { Ayyaswamy (1990) }\end{array}$ & $\begin{array}{c}\text { numerical } \\
\text { fully }\end{array}$ & yes & no & yes \\
\hline $\begin{array}{c}\text { Chiang et al. } \\
\text { (1992) }\end{array}$ & numerical
\end{tabular}

Table 6-1. Some of the previous theoretical work on convective droplet evaporation and burning. 

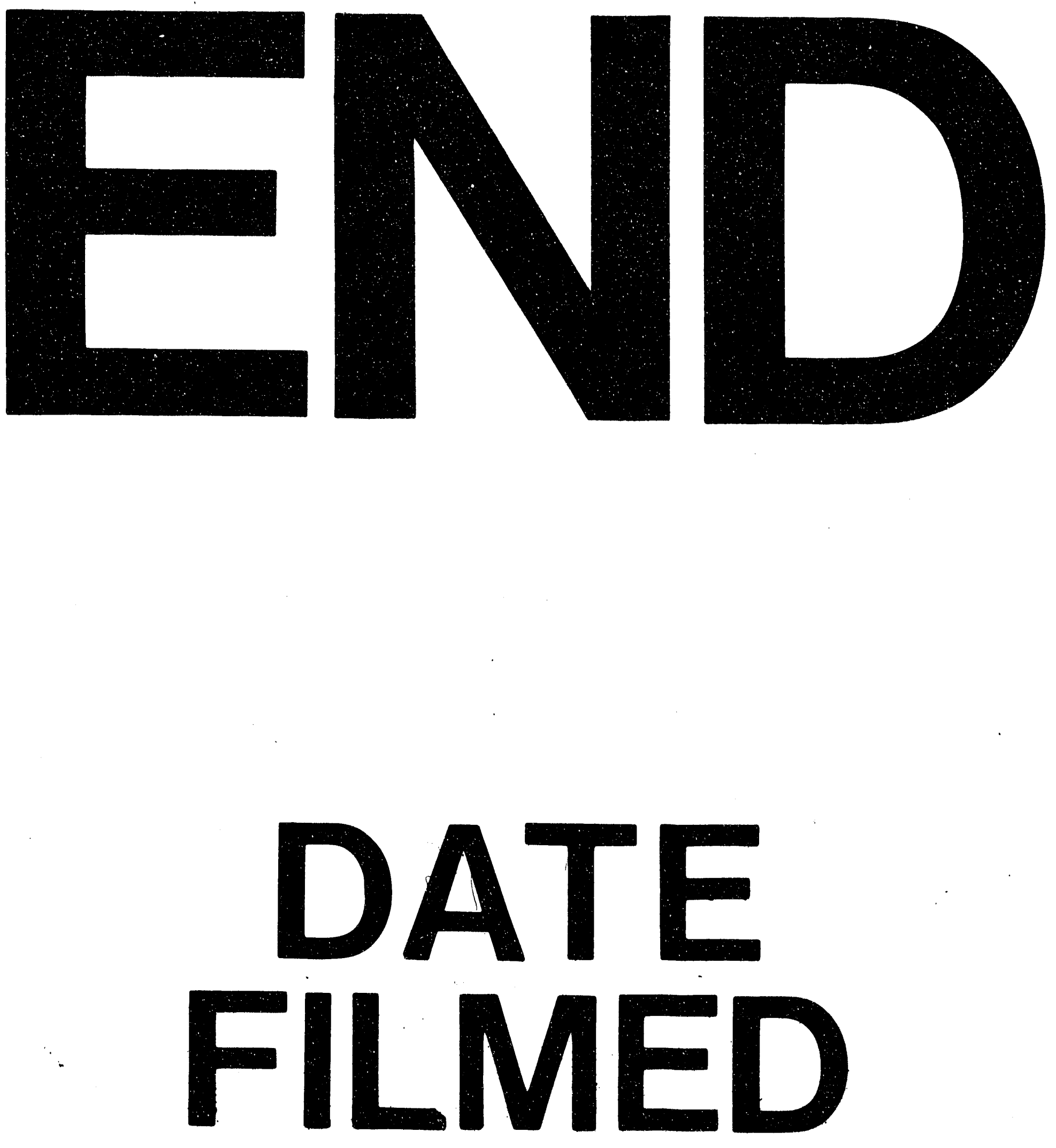

.

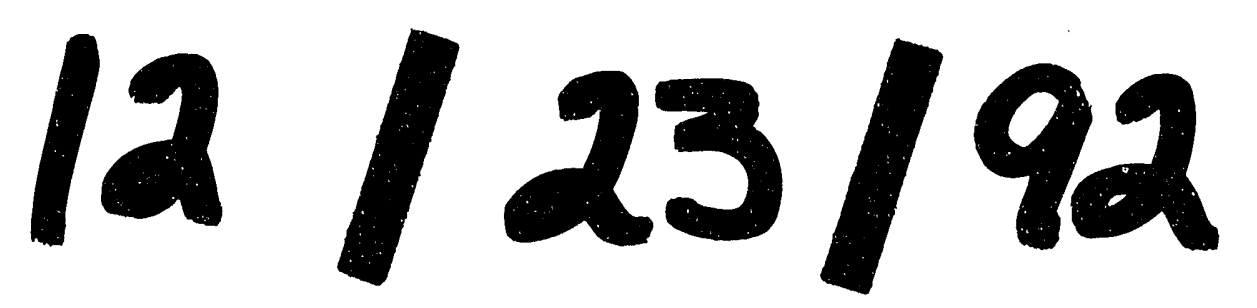


